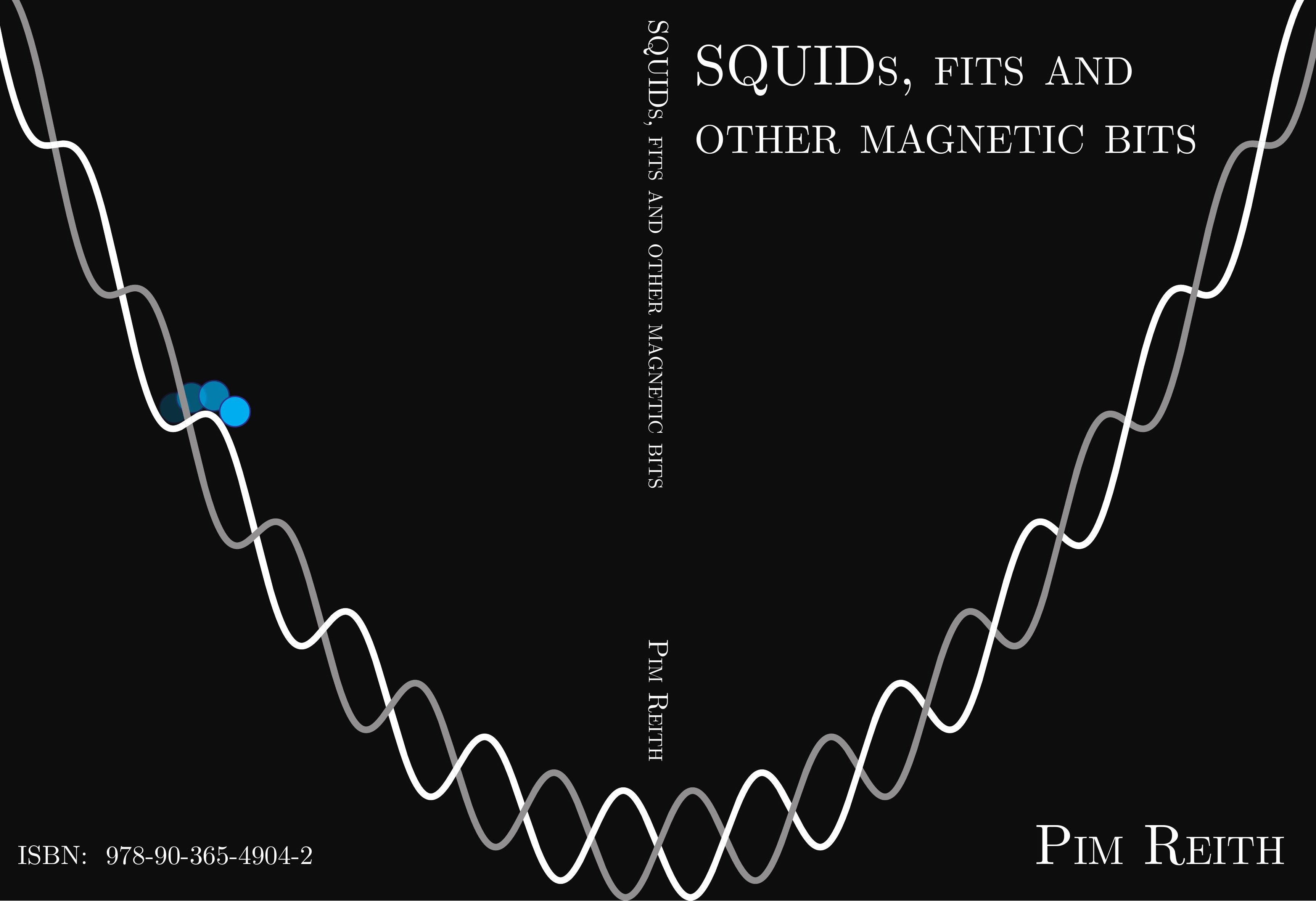




\section{SQUIDS, FITS AND \\ OTHER MAGNETIC BITS}

Pim Reith 



\title{
SQUIDS, FITS AND OTHER MAGNETIC BITS
}

\author{
DISSERTATION
}

to obtain

the degree of doctor at the University of Twente on the authority of the Rector Magnificus, prof. dr. T.T.M. Palstra, on account of the decision of the Doctorate Board, to be publicly defended on Thursday the $5^{\text {th }}$ of December, 2019, at 10:45 by

\section{Pim Reith}

born on the $23^{\text {rd }}$ of February, 1991 in Beuningen, the Netherlands 
This thesis has been approved by the promotors:

Prof. dr. ir. J.W.M. Hilgenkamp

Prof. dr. ir. W.G. van der Wiel

The research in this thesis was performed mainly at the MESA+ Institute for Nanotechnology at the University of Twente. It was funded through the DESCO programme of the Dutch Research Council (NWO).

The cover displays the two solutions of the free energy of a dc SQUID with a built-in $\pi$-shift.

Printed by: Gildeprint - Enschede

ISBN: $\quad$ 978-90-365-4904-2

DOI: $\quad 10.3990 / 1.9789036549042$

(C)2019 P. Reith, the Netherlands. All rights reserved. No parts of this thesis may be reproduced, stored in a retrieval system or transmitted in any form or by any means without permission of the author.

Alle rechten voorbehouden. Niets uit deze uitgave mag worden vermenigvuldigd, in enige vorm of op enige wijze, zonder voorafgaande schriftelijke toestemming van de auteur. 


\section{Graduation committee}

Chair and secretary

Prof. dr. J.L. Herek

University of Twente

Supervisors

Prof. dr. ir. J.W.M. Hilgenkamp

University of Twente

Prof. dr. ir. W.G. van der Wiel

University of Twente

\section{Members}

Prof. dr. J. Aarts

Leiden University

Prof. dr. P.A. Bobbert

Prof. dr. ir. G. Koster

Prof. dr. F. Lombardi

Prof. dr. ir. H.J.W. Zandvliet

Eindhoven University of Technology and University of Twente University of Twente Chalmers University of Technology University of Twente 

The meaning of life is to give life meaning. - A.A. Lucassen 



\section{Contents}

1 Magnetism: current and spin 1

1.1 Magnetism from currents . . . . . . . . . . . . . . 2

1.2 Magnetism from spin . . . . . . . . . . . . . . . 3

1.3 Measuring magnetism . . . . . . . . . . . 5

$\begin{array}{lll}2 & \text { Scanning SQUID microscopy } & 7\end{array}$

2.1 The dc SQUID . . . . . . . . . . . . . . . . . . 8

2.2 Scanning SQUID microscopy $\ldots \ldots \ldots \ldots$

2.3 Modelling of superconducting vortices . . . . . . . . . . 19

2.4 Modelling of ferromagnetic surfaces . . . . . . . . . . 21

2.5 Discussion and conclusion . . . . . . . . . . . . 25

3 Scanning SQUID microscopy and magnetometry on low$T_{c} /$ high- $T_{c}$ hybrid junctions $\quad \mathbf{2 7}$

3.1 Introduction . . . . . . . . . . . . . . 28

3.2 Spontaneous half flux quanta in $\pi$-loops $\ldots \ldots$. . . . . . 29

3.3 Influence of the loop inductance . . . . . . . . . . . . . 31

3.4 Design and fabrication of $\pi$-loops . . . . . . . . . . . 33

3.5 Measurements on Nb-based hybrid junctions . . . . . . . . . 34

3.6 Measurements on MoRe-based hybrid junctions . . . . . . . 40

3.7 Discussion and conclusion . . . . . . . . . . . . . . 44

4 Scanning SQUID microscopy on dipole-based systems 49

4.1 Dipole theory . . . . . . . . . . . . . 50

4.2 Magnetic pollution . . . . . . . . . . . . . . 51

4.3 Measurements on $\mathrm{Fe}_{3} \mathrm{Se}_{4}$ nanoparticles . . . . . . . . . 53

4.4 Palaeointensity measurements . . . . . . . . . . . 56 
4.5 Concluding remarks . . . . . . . . . . . . . 64

5 Critical thickness behaviour in $\mathrm{LaMnO}_{3}$ thin films 65

5.1 Introduction . . . . . . . . . . . . . . . . . . 66

5.2 Sample fabrication . . . . . . . . . . . . 67

5.3 X-ray absorption spectroscopy . . . . . . . . . . . 68

5.4 Hard X-ray photoemission spectroscopy . . . . . . . . . 69

5.5 X-ray magnetic circular dichroism . . . . . . . . . . 70

5.6 Scanning transmission electron microscopy . . . . . . . . 72

5.7 Vibrating sample magnetometry . . . . . . . . . . 75

5.8 Scanning SQUID microscopy . . . . . . . . . . . 76

5.9 Discussion and conclusion . . . . . . . . . . . . . 77

6 Critical thickness behaviour in $\mathrm{LaMnO}_{3} / \mathrm{LaAlO}_{3}$ heterostructures $\quad 81$

6.1 Introduction . . . . . . . . . . . . . . . . . . 82

6.2 Fabrication . . . . . . . . . . . . 83

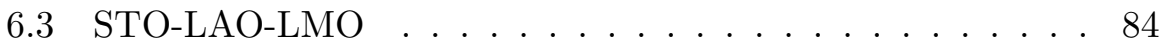

6.4 STO-LMO-LAO . . . . . . . . . . . . . 88

6.5 Discussion and conclusion . . . . . . . . . . . . 91

7 Designing scanning SQUID experiments on topologically non-trivial materials $\quad 95$

7.1 Introduction . . . . . . . . . . . . . . 96

7.2 Edge currents . . . . . . . . . . . . . . . . . 996

7.3 Spin-momentum locking . . . . . . . . . . . . . . 98

$7.4 p$-wave superconductivity . . . . . . . . . . . . 100

7.5 Magnetic monopole image charge . . . . . . . . . . . 106

7.6 Concluding remarks . . . . . . . . . . . . . 108

8 Closing thoughts 111

8.1 Concluding remarks on this thesis . . . . . . . . . 112

8.2 On the limits and future of scanning SQUID microscopy technology . . . . . . . . . . . . . . . . . . . . 114

$\begin{array}{lr}\text { Samenvatting } & 119\end{array}$

$\begin{array}{ll}\text { Summary } & 123\end{array}$ 
Acknowledgements

List of publications 



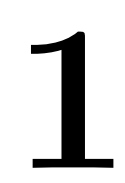

\section{Magnetism: current and spin}

Magnetism has played an important role for much of human history. Lodestone, a piece of naturally magnetised iron oxide, was known to several ancient civilisations and used for purposes such as divination. The oldest descriptions of lodestone date back to the $6^{\text {th }}$ century BCE in ancient Greece and the $4^{\text {th }}$ century BCE in China, though there is evidence that the Olmec civilisation in Mesoamerica were aware of its properties even before that [1]. The term 'magnet' is also believed to be derived from the ancient Greek term $\mu a \gamma \nu \tilde{\eta} \tau \iota \varsigma$ 人ivos (magnetis lithos, or 'stone of Magnesia'), named after the Greek city Magnesia in modern-day Turkey where these stones could be found.

The Chinese likely invented the navigational compass around the early $11^{\text {th }}$ century CE [2]. Almost a millennium later, in 1819, it was the compass that showed Ørsted a possible link between electricity and magnetism. This paved the way for a unified theory of electromagnetism culminating in Maxwell's equations, which in turn formed part of the foundation on which Einstein built his theory of special relativity. Soon after, with the development of quantum mechanics, science was able to explain the origin of ferromagnetism, finally revealing why such a magical material as lodestone could even exist.

These days, magnets can be found everywhere in our daily lives, from fridge magnets to electric guitars to MRI scanners. With modern society's focus on not only improved performance, but energy efficiency and sustainability as well, both fundamental and technological research on magnetism is as alive as ever. This work contains a collection of experi- 
ments on different materials and devices: superconducting devices, ferromagnetic particles and thin films, even palaeomagnetic samples. We do this mostly using a scanning superconducting quantum interference device microscope, which we will introduce later on.

\subsection{Magnetism from currents}

Maxwell's equations show that electric and magnetic fields are two sides of the same coin, and Einstein proved that these fields transform into one another upon changing the reference frame. Two of Maxwell's equations describe the behaviour of the magnetic field $B$ :

$$
\begin{aligned}
\nabla \cdot B & =0, \\
\nabla \times B & =\mu_{0}\left(J+\epsilon_{0} \frac{d E}{d t}\right),
\end{aligned}
$$

where $\mu_{0}$ is the magnetic permeability of the vacuum, $\epsilon_{0}$ is the electric permittivity of the vacuum, $J$ is the electric current density, $t$ is time and $E$ is the electric field.

Unlike its electric counterpart, there appears to be no fundamental magnetic charge in nature (Equation 1.1). From Maxwell's equations, we see that only a current and a dynamic electric field can create a magnetic field. Assuming the electric field to be constant for now $(d E / d t=0)$, we can describe the magnetic field vector $\vec{B}$ using the Biot-Savart law:

$$
\vec{B}=\frac{\mu_{0}}{4 \pi} \oint_{C} \frac{I \delta \vec{\ell} \times \vec{r}}{r^{3}}
$$

where $I$ is the total current and $\vec{r}$ is the vector from a point $d \vec{l}$ on the current path $C$ to the point of interest.

While most of the studies in this work are on ferromagnetic materials, current-based magnetic fields are a crucial ingredient to the functionality of the devices in Chapter 3. These fields will also be discussed and used to model some effects in Chapters 2 and 7. 


\subsection{Magnetism from spin}

The second source of magnetic fields is the spin magnetic moment. Although magnetism in solids was first believed to be due to tiny circulating currents (the Ampère model), quantum mechanics showed that spin is an even more fundamental property of matter than that.

\subsubsection{The magnetic moment}

The relation between spin $S$ and magnetic moment $m$ is given by

$$
m=-\frac{g \mu S}{\hbar},
$$

where $g$ is the g-factor, $\hbar$ is the reduced Planck's constant and $\mu$ the magneton, defined as

$$
\mu=\frac{e \hbar}{2 m_{p}},
$$

where $e$ is the elementary charge and $m_{p}$ the mass of the particle.

The $\mathrm{g}$-factor is a dimensionless constant and has different values for different particles. $g \approx 2$ for electrons, $g \approx 5.6$ for protons and $g \approx-3.8$ for neutrons. From the above, we can immediately see that the magnetic moment of a nuclear spin is much smaller than that of an electron spin, since a nuclear mass is roughly $10^{3}$ times larger. Because of this, we can reasonably neglect the contribution of the nucleus to the total magnetic moment of a material.

For electrons, then, we get

$$
\mu_{S}=-\frac{g_{e} \mu_{B} S}{\hbar}
$$

where $g_{e} \approx 2$ is the electron spin $g$-factor, $S= \pm \frac{\hbar}{2}$ is the spin angular momentum and $\mu_{B}=\frac{e \hbar}{2 m_{e}}=9.274 \times 10^{-24} \mathrm{~J} \cdot \mathrm{T}^{-1}$ is the Bohr magneton. From this, we see that $\mu_{S} \approx \mu_{B}$.

The orbital magnetic moment is due to the electron orbiting the nucleus. This can be viewed as a charged particle travelling around a loop, thereby producing a magnetic field. The orbital magnetic moment $\mu_{L}$ is given by

$$
\mu_{L}=-\frac{g_{L} \mu_{B} L}{\hbar}
$$


where $g_{l} \approx 1$ is the orbital $g$-factor and $L$ is the orbital angular momentum.

In most atoms, the majority of electrons are paired in their respective orbitals, meaning that they have opposite spins. This causes most of the magnetic moment to be cancelled out. It is only the unpaired electrons that will lead to a net magnetic moment on an atom.

\subsubsection{Ferromagnetism}

While an atom can have a net spin, that does not necessarily mean a material will have a non-zero magnetic moment. In most materials, the spins are randomly oriented, meaning they cancel out when averaged over a larger volume. Spins will align only when an external magnetic field is applied, aligning parallel (paramagnetism) or antiparallel (diamagnetism) to said field. Upon removing the field, the spins will again orient themselves randomly due to their thermal energy.

The interaction between magnetic moments on neighbouring atoms is dominated by the exchange interaction. While the magnetic field produced by an atom creates a preferential orientation for a neighbouring moment, the energy difference associated with this is on the order of $\mu \mathrm{eV}$ [3]. In most experiments, the thermal energy $k_{B} T$ will be much larger, which would mean neighbouring magnetic moments will not align.

The exchange interaction, however, results in a much stronger coupling. It arises due to the antisymmetric nature of electron wave functions. That is, if two electrons have the same spatial wave function (e.g. in a bonding orbital), their spin wave functions must be opposite. Conversely, if their spatial wave functions are opposite (e.g. in an antibonding orbital), then their spin wave functions must be the same. Antisymmetric spatial wave functions will have a larger expectation value of the separation between the two electrons. Therefore, an antisymmetric wave function lowers the energy associated with the Coulomb interaction between the electrons. This phenomenon, called direct exchange, can create a preference for having aligned spins.

In most materials, direct exchange will not lead to ferromagnetism. This can be because the orbitals in question are very localised (e.g. $4 f$ orbitals), meaning the overlap from atom to atom is very small. A small overlap can also be caused by atoms being separated by a different element (e.g. Mn atoms being separated by an oxygen atom in $\mathrm{MnO}$ ). In such materials, other (indirect) exchange interactions may still provide a means 
to cause ferromagnetic alignment.

In this thesis, ferromagnetic materials in the form of individual microscopic particles and thin films will be studied in Chapters 4, 5 and 6, with some additional modelling performed in Chapter 2.

\subsection{Measuring magnetism}

Humans have no innate sensitivity to magnetic fields, unlike some other species in nature. Most famously, birds use it to navigate during seasonal changes. Some bacteria species, known as magnetotactic bacteria, have an awareness of magnetic fields as well. However, us humans must resort to measurement equipment to visualise magnetic fields.

Typical studies on ferromagnetic materials revolve around the hysteresis loop, where some parameter is measured as a function of an external magnetic field. Ferromagnets have multiple stable states, which can be changed by applying a field. However, changing from one state to another usually involves crossing some energy barrier, which is why it typically requires a non-zero field to rotate the spins. Thus, hysteresis appears.

For example, a regular magnetometry measurement can be used to measure the magnetic field produced by the sample itself. This can then be used to estimate the number of spins that each magnetic atom contributes, which can be a measure of sample quality or may be used to study more fundamental aspects of the material. The coercive field (the field where the sample switches from one state to another) is related to the height of the energy barrier between different states, which is a measure of magnetic domain size and magnetic anisotropy.

More advanced measurements involve diffraction and absorption studies, typically based on X-rays or neutrons. Depending on the polarisation of the X-ray, it will be absorbed differently by certain spin configurations in a material. This means that measuring the difference between the absorption spectra of X-rays with different polarisations can yield information on the spin state of magnetic atoms. Neutron diffraction, on the other hand, can be used to determine the long-range ordering of spins in a material, which was crucial in the discovery of antiferromagnetism.

Lastly, there are local measurement techniques. These usually take the form of scanning probe techniques, of which scanning tunnelling microscopy and magnetic force microscopy are the most common. These techniques allow for imaging the magnetic response of a sample down to 
the smallest scales, which enables us to study the properties of individual magnetic domains, domain walls, or even more complicated magnetic structures such as superconducting vortices and skyrmions.

Among these scanning probe techniques is scanning superconducting quantum interference device microscopy (scanning SQUID microscopy, or SSM for short). At the heart of the SSM is the SQUID, a piece of superconducting circuitry that enables the highest magnetic field sensitivity to date. By scanning such a SQUID over a surface, we can directly image the magnetic activity on that surface and measure the magnetic field strengths involved. This allows us to not only see visually what is going on, but also use the data in various magnetic field models to extract interesting parameters of the sample.

The scanning SQUID microscope forms the core of this work. In Chapter 2, we will have a more detailed look at the setup itself, as well as various aspects of imaging and analysis. In most of the following text, Chapters 3 through 6 , we will use the SSM, in combination with other techniques, to explore different materials and devices. Finally, in Chapter 7, we will spend a little time to look at a class of materials, topological non-trivial matter, and see where the SSM can fit in to study their properties. 


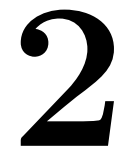

\section{Scanning SQUID microscopy}

This chapter will describe the principles of Scanning SQUID Microscopy (SSM), as well as details pertaining to the setup used in the experiments throughout this thesis. In addition, we will discuss various aspects of imaging using SSM through numeric modelling.

I would like to thank Dr. Xiao Renshaw Wang for his contributions to this chapter. Parts of this chapter have been published as P. Reith et al., Analysing magnetism using scanning SQUID microscopy, Review of Scientific Instruments volume 88, 123706, 2017. 


\subsection{The dc SQUID}

Scanning SQUID microscopy is a member of the scanning probe microscopy family. It images the magnetic field emanating from a sample surface using a direct-current Superconducting Quantum Interference Device (dc SQUID), an extremely sensitive magnetometer. The SQUID is based on the concept of fluxoid quantisation, which will be explained below.

\subsubsection{Flux quantisation}

A superconductor is commonly described by using a wave function $\Psi(r, t)$ :

$$
\Psi(\vec{r}, t)=|\Psi(\vec{r}, t)| e^{i \theta(\vec{r}, t)},
$$

where $|\Psi(\vec{r}, t)|$ is the amplitude and $\theta(\vec{r}, t)$ the phase of the wave function at a position $\vec{r}$ and time $t$. As a wave function, it must obey the Schrödinger equation for a charged particle:

$$
i \hbar \frac{\partial \Psi}{\partial t}=\left[\frac{1}{2} m\left(\frac{\hbar}{i} \nabla-q \vec{A}\right)^{2}+q \phi\right] \Psi .
$$

Here, $m_{p}$ and $q$ are the mass and charge of the charge carrier (in our case, a Cooper pair), $\hbar$ is the reduced Planck's constant, $\vec{A}$ is the magnetic vector potential and $\phi$ the electric scalar potential. From this, a relation between $\theta, \vec{A}$ and the supercurrent density $\vec{J}_{s}$ can be obtained (the full derivation can be found elsewhere [4]):

$$
\nabla \theta=2 \pi\left[\frac{2 m_{e}}{h n_{s} e} \vec{J}_{s}+\frac{2 e}{h} \vec{A}\right],
$$

where $m_{e}$ is the mass of the electron, $h$ is Planck's constant and $n_{s}$ the Cooper pair density.

Now imagine we have a superconducting ring, through which there is some amount of magnetic flux (Figure 2.1A). To find out the details of this flux, we can integrate Equation 2.3 along a closed path $C$ around the ring:

$$
\oint_{C} \nabla \theta \cdot d \vec{\imath}=\frac{4 \pi e}{h}\left[\frac{m_{e}}{n_{s} e^{2}} \oint_{C} \vec{J}_{s} \cdot d \vec{\imath}+\oint_{C} \vec{A} \cdot d \vec{l}\right]
$$



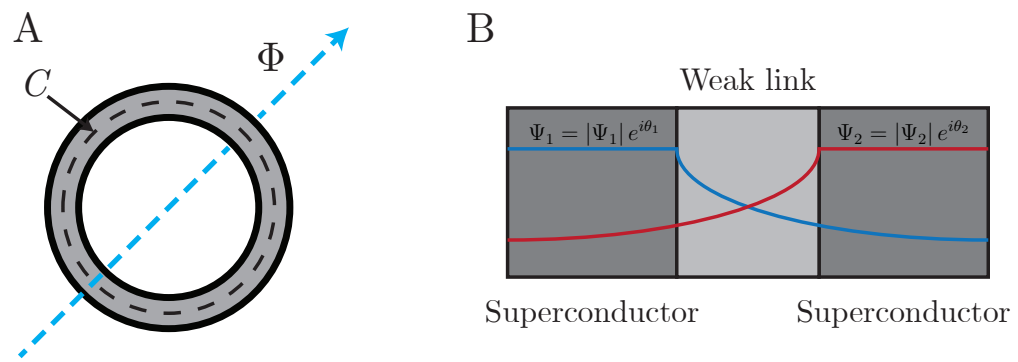

Figure 2.1: A) Schematic depiction of a superconducting ring threaded by a flux $\Phi$. B) Schematic depiction of a Josephson junction.

The term on the left-hand side of the equation is simply the total phase difference $\Delta \theta$ along the closed loop. Since $\Psi$ must be single-valued, we have $\Delta \theta=n \cdot 2 \pi$. On the right-hand side, we can apply Stokes' theorem to find

$$
\oint_{C} \vec{A} \cdot d \vec{l}=\int_{S} \vec{B} \cdot d \vec{S}
$$

where $\vec{S}$ is the area enclosed by $C$ and $\vec{B}$ is the magnetic flux density. Note that the right-hand side is exactly the definition for the magnetic flux $\Phi$. Now, if we choose $C$ to be deep enough in the superconductor (i.e., at a distance $d \gg \lambda$ from the edge), then the magnetic flux at that point will be zero and therefore $\vec{J}_{s}=0$ as well. We can then combine all of the above to find

$$
\begin{aligned}
2 \pi n & =\frac{4 \pi e}{h} \Phi, \\
\Phi & =n \frac{h}{2 e} .
\end{aligned}
$$

Therefore, the total flux through the ring (i.e., the sum of all external and self-generated flux) is quantized in units of $h / 2 e=2.0678 \times 10^{-15} \mathrm{~T} \cdot \mathrm{m}^{2}$. For this reason, $h / 2 e$ is also defined as the superconducting flux quantum $\Phi_{0}$.

\subsubsection{Josephson junctions and fluxoid quantisation}

When two superconductors are connected through a so-called weak link, the result is a Josephson Junction. A weak link can be created in various 
ways but is commonly done using either a constriction or a thin barrier of non-superconducting material. In a Josephson junction, Cooper pairs are able to tunnel across the weak link from one superconductor into the other (see Figure 2.1B).

The current $I$ through and the voltage $V$ across Josephson Junctions are described by the two Josephson equations:

$$
\begin{aligned}
I & =I_{c} \sin \varphi, \\
V & =\frac{\hbar}{2 e} \frac{d \varphi}{d t},
\end{aligned}
$$

where $I_{c}$ is the critical current and $\varphi=\theta_{1}-\theta_{2}$ is the difference between the phases of the wave functions of the two superconductors as shown in Figure 2.1B.

If we were to create a superconducting circuit with a Josephson junction, or another element that can cause an extra phase shift, the earlier flux quantisation equation has to be modified to include this change. This means that Equation 2.4 becomes

$$
2 \pi n=\frac{4 \pi e}{h}\left[\frac{m}{n_{s} e^{2}} \oint_{C} \overrightarrow{J_{s}} \cdot d \vec{\imath}+\oint_{C} \vec{A} \cdot d \vec{l}\right]+\sum_{i=1}^{N} \varphi_{i},
$$

where $\varphi_{i}$ is the phase drop associated with some circuit element $i$. Rather than flux (as seen in Equation 2.7), it is now this expanded right hand side, known as a fluxoid, that has to be quantized.

\subsubsection{Direct-current SQUIDs and the flux-locked loop}

A dc SQUID is obtained when two Josephson Junctions are added to a superconducting ring, shown in Figure 2.2A. For a SQUID, the fluxoid quantisation equation reduces to

$$
n=\frac{1}{2 \pi}\left(\varphi_{1}+\varphi_{2}\right)+\frac{\Phi}{\Phi_{0}},
$$

where $\varphi_{1}$ and $\varphi_{2}$ are the phase drops across the two Josephson Junctions. If we assume that the critical current through both junctions is the same (i.e., $I_{\mathrm{c} 1}=I_{\mathrm{c} 2}=I_{c}$ ), then we can write the total current through the circuit as

$$
I=I_{c}\left[\sin \left(\varphi_{1}\right)+\sin \left(\varphi_{2}\right)\right]
$$



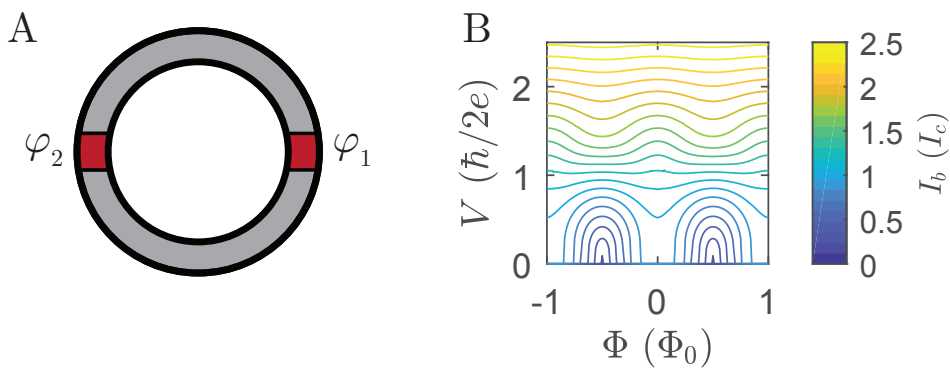

Figure 2.2: A) dc SQUID made out of a superconducting ring with 2 Josephson junctions (red). B) SQUID voltage $V$ as function of the magnetic flux $\Phi$ for different values of the bias current $I_{b}$.

which, when combined with the fluxoid quantisation condition, leads to

$$
I_{\mathrm{SQUID}}=2 I_{c}\left|\cos \pi \frac{\Phi}{\Phi_{0}}\right|,
$$

where $I_{\text {SQUID }}$ is the critical current of the SQUID. From Equation 2.13, we can see that $I_{\text {SQUID }}$ is directly related to the total flux $\Phi$ through the SQUID. This direct dependence enables the dc SQUID to be a useful magnetometer.

However, Equation 2.13 is not useful in practice: determining the flux during a measurement would require measuring $I_{\text {SQUID }}$. This is done through ramping the current from zero until $I_{\text {SQUID }}$ is reached for every measurement point. While this might be a feasible approach for slow measurements in which $\Phi$ does not vary significantly, it is not practical for use in a scanning probe microscope where faster acquisition is needed.

For this reason, dc SQUIDs are often operated in the voltage state. Using an external bias current $I_{b}$, the SQUID is biased just above the critical current. There, the voltage $V$ across the SQUID is directly related to the flux $\Phi$. The relation between the two is depicted in Figure 2.2B. As we can see, it resembles a sinusoidal function for $I_{b}>2 I_{c}$ with a period of $\Phi_{0}$. To make use of the SQUID, we can attempt to linearise the relation using a feedback system. In such a setup, a modulation coil is added around the SQUID which can apply an external flux $\Phi_{m}$ to the SQUID. This allows us to choose a working point on the flux-voltage relation, typically at the point where $\frac{d V}{d \Phi}$ is largest.

When an external flux $\Phi_{e}$ threads the loop, it moves the system off the working point. By changing the modulation current that controls $\Phi_{m}$, the 
system is moved back to the working point. The increase or decrease to the modulation current is then a measure for $\Phi_{e}$. Since the feedback system is trying to keep the SQUID at the working point, which means maintaining a constant flux through the SQUID, such a setup is often called a fluxlocked loop. Although several variations on this concept exist, the basic idea is always to maintain a working point by using a modulation coil.

\subsection{Scanning SQUID microscopy}

\subsubsection{SQUID sensor}

To use a SQUID sensor in scanning SQUID microscopy, the SQUID is often extended with a pickup loop as shown in Figure 2.3. Such a pickup loop is usually lithographically defined with as small an area as possible, since the size of the pickup loop is directly related to the spatial resolution (discussed below). A pickup loop can either be directly connected to the SQUID itself (Figure 2.3A) or be inductively coupled (Figure 2.3B).

A

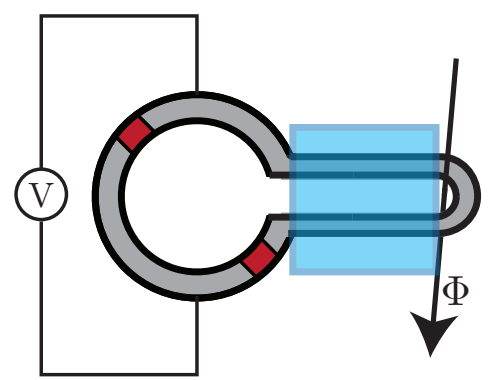

$\mathrm{B}$

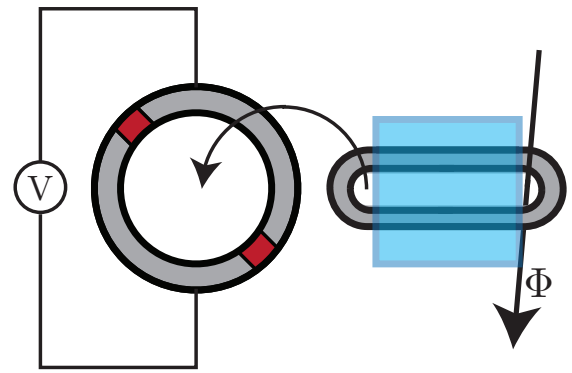

Figure 2.3: SQUID extended with a pickup coil, either A) directly or B) inductively. The pickup loop is defined partially by magnetic shielding (light blue).

Next, the SQUID is brought in close proximity to the sample surface. Usually, the SQUID sensor is fixed in space on the setup to remove any influence from gradients in the local (lab) magnetic field. Instead, the sample can be moved in three dimensions to provide the scanning motion required for imaging. In this thesis, we define the sample surface to be the $x y$-plane and the perpendicular direction to be the $z$-axis.

The SQUID outputs a voltage signal through the flux-locked loop while scanning. The voltage $V$ is converted to a magnetic flux $\Phi$ or magnetic 
field $B$ by noting that

$$
\Phi=B A_{s}=\frac{\Phi_{0}}{F} V
$$

where $F$ is the transfer ratio between voltage and flux in units of $\Phi_{0}$ and $A_{s}$ is the area of the pickup loop. $F$ is obtained through a calibration step before scanning.

For proper conversion to magnetic field values (often preferable over flux for comparison with theoretical models), $A_{s}$ should be known accurately. Although the dimensions of the pickup loop are defined in the lithography masks used during fabrication, errors during mask alignment can cause $A_{s}$ to vary slightly between sensors. In addition, while the physical dimensions of the pickup loop can easily be measured using optical microscopy (or electron microscopy for sub-micron pickup loop sizes), additional effects like flux focusing can cause the effective area to be larger than the physical.

Determining the value of $A_{s}$ can be done in one of two ways. Computer modelling of the effective area based on the physical width of the pickup loop can yield an accurate answer. Such analyses exist [5-7], but they require an optimized model and computing time.

The other method is to measure the area experimentally. In the past, this was done by calibrating the sensor area against a known magnetic object, usually an Abrikosov vortex. By imaging an Abrikosov vortex, which carries a total magnetic flux of $1 \Phi_{0}$, the value of $A_{s}$ can be adjusted until the measurement matches the predicted values for the field. Through this method, we find that our sensor has $A_{s} \approx 10 \mu^{2}$. The accuracy of and drawbacks to this method will be discussed in more detail in Section 2.3 .

A second method would be to measure the SQUID response to an externally applied magnetic field. By setting up the sensor in a fluxlocked loop as usual, we can vary an external field over a range $\Delta B$ and measure the linear response $\Delta V$. From Equation 2.14, we then find

$$
A_{s}=\frac{\Phi_{0}}{F} \frac{\Delta V}{\Delta B}
$$

The magnetic field can be applied in two ways: either by using a large external magnet or by creating a local magnetic field (e.g., by fabricating a small coil or current line on a sample). The first method would give 
a more accurate value for the applied field. However, at least with our own sensor design, the field will then also go through the SQUID washer, yielding a much larger flux than from just the pickup loop alone. This would then measure the entire exposed area, not just $A_{s}$.

The second method solves this problem if the pickup loop and the SQUID washer are separated by a significant amount (in our case, approximately $800 \mu \mathrm{m}$ ). The problem with this method is that the magnetic field produced will be less homogeneous, which makes it difficult to precisely determine the magnetic field that is penetrating the pickup loop.

\subsubsection{Spatial resolution}

In simple terms, spatial resolution is the smallest distance between two points that an imaging device can resolve. One way to quantify this was given by Kirtley et al., who used a definition derived from the Rayleigh criterion commonly used in optics [8]. However, most papers forego assigning a value to the spatial resolution and only mention the setup geometry. We define the spatial resolution here as the distance $s$ between the two extreme values of the field of an in-plane point dipole (Figure 2.4A-B). We choose this definition because with infinitely good resolution, the two extrema should have, in theory, an infinitesimal separation. Additionally, we believe this to be a practical definition: it should be relatively easy to measure a particle with a dipolar magnetic field much smaller than the spatial resolution, thereby experimentally establishing the resolution of the system.

The value of $s$ depends on the sensor height $h^{1}$ and the pickup loop diameter $d$. It can be calculated through the following implicit equation (derived in [9]):

$$
\frac{s+d}{\left(\left(\frac{s+d}{2}\right)^{2}+h^{2}\right)^{\frac{5}{2}}}=\frac{s-d}{\left(\left(\frac{s-d}{2}\right)^{2}+h^{2}\right)^{\frac{5}{2}}} .
$$

Figure 2.4C shows $s$ as a function of $h$, both normalised to $d$. We see that for large $h / d$, we get $s \approx d$, and similarly for small $h / d$ we find $s \approx h$. In the intermediate region, where $h / d$ is on the order of unity, we find that $s$ is larger than either.

\footnotetext{
${ }^{1}$ From this point forward, $h$ will denote the sensor height in this thesis and not Planck's constant.
} 

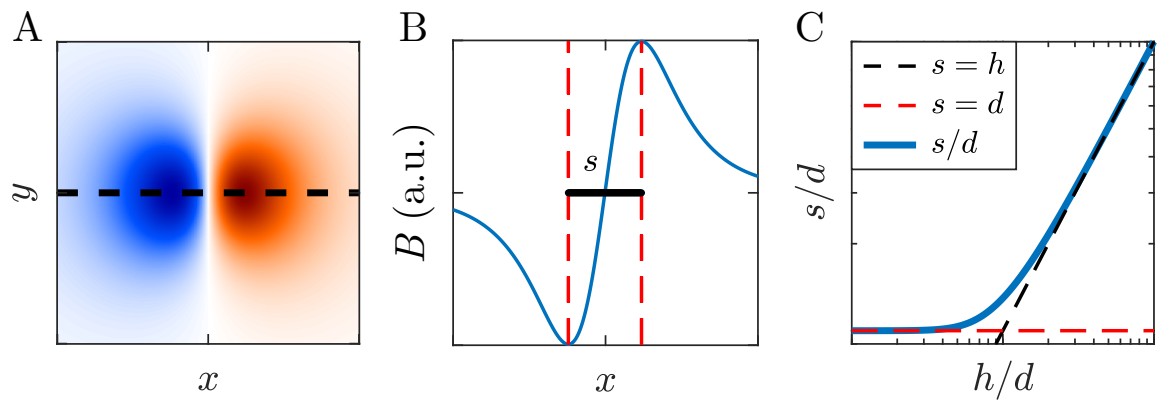

Figure 2.4: Definition of spatial resolution based on a point-dipole. A) Simulated out-of-plane field image of a point-dipole at the origin with a magnetic moment aligned along the $x$-axis. B) Magnetic field profile indicated in (A). The red dashed lines indicate the two extrema, with the separation distance $s$ indicated by the black solid line. C) The normalised resolution $s / d$ as a function of $h / d$ (blue solid line), with the limits $s=d$ (red dashed) and $s=h$ (black dashed).

Using this definition, the spatial resolution of an SSM system can be determined by imaging a dipole which is smaller than the resolution of the system and so can be considered a point dipole. Ferromagnetic nanoparticles are particularly suitable for this. Using estimated values of $h$ and $d$ for our own system, the resolution turns out to be approximately $10 \mu \mathrm{m}$. An experimental verification of the spatial resolution is presented in Section 4.3.3.

\subsubsection{Scanning angle}

In our setup, the sensor is positioned at a $27^{\circ}$ angle. This ensures that the sensor tip with the pickup loop is closest to the sample during measurements. Because of this, measurement data will be deformed. This can be visualized by looking at the field of an Abrikosov vortex (here modelled as a monopole for simplicity [8]):

$$
\vec{B}=\frac{\Phi_{0}}{2 \pi r^{3}} \vec{r}
$$

The SQUID sensor is sensitive to the magnetic field component perpendicular to the sensor plane. Ideally, the sensor plane is the $x y$-plane and therefore the SQUID is only sensitive to the $z$-component of the magnetic 
A

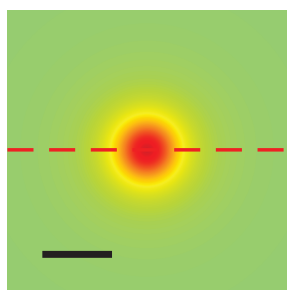

B

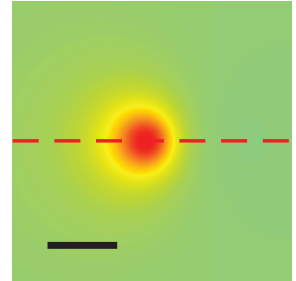

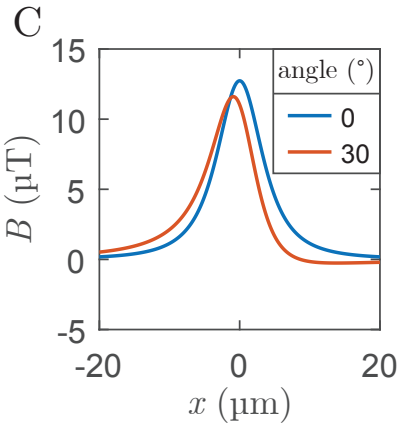

Figure 2.5: Influence of the scanning angle. A-B) Simulated image of an Abrikosov vortex with the sensor A) parallel to and B) under a $30^{\circ}$ angle with the $x y$-plane. Scale bars indicate $10 \mu \mathrm{m}$. C) Single line indicated in A and B.

field. In the case of a non-zero angle $\theta$ with the $x$-axis, this becomes

$$
B=\frac{\Phi_{0}}{2 \pi r^{3}}[x \sin \theta+z \cos \theta],
$$

Figure 2.5A shows a simulation of how a vortex would be imaged by an $\mathrm{SSM}$ with the sensor in the $x y$-plane, at a height $h=5 \mu \mathrm{m}$. As expected, the vortex is circularly symmetric. Figure $2.5 \mathrm{~B}$ shows the same vortex, but this time as it would be imaged with the sensor under a $30^{\circ}$ angle (similar to the angle in our setup design). We can see that the image appears slightly 'smeared' to the left side, a consequence of part of the $x$ component of the field being measured. Figure 2.5C shows a comparison of the two lines drawn in $2.5 \mathrm{~A}$ and $\mathrm{B}$, highlighting the influence of the angle on the magnetic field profile.

To determine the sensor angle, we performed two different experiments. The first is shown in Figure 2.6A, where we imaged an Abrikosov vortex in a $100 \mu \mathrm{m} \times 100 \mu \mathrm{m} \mathrm{Nb}$ square. We can clearly see both the vortex and the fact that it appears smeared towards the left. As shown above, this is due to the sensor being at an angle. Additionally, we can see flux focusing just outside the top, bottom and right edge of the Nb square, but not near the left edge. Because of the sensor angle, the field lines on the left edge run more parallel to the sensor plane, obscuring the flux focusing effect.

A single line of the data in Figure 2.6A is shown in Figure 2.6B. Using equation 2.18, we can make a fit to the data to extract the scanning angle. This fit is shown in Figure 2.6B and yields an angle of approximately $40^{\circ}$, 
A

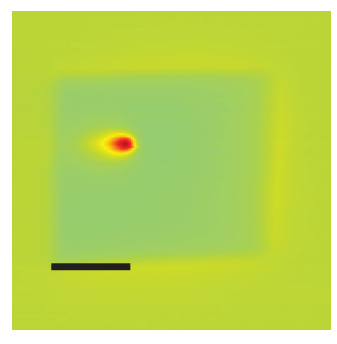

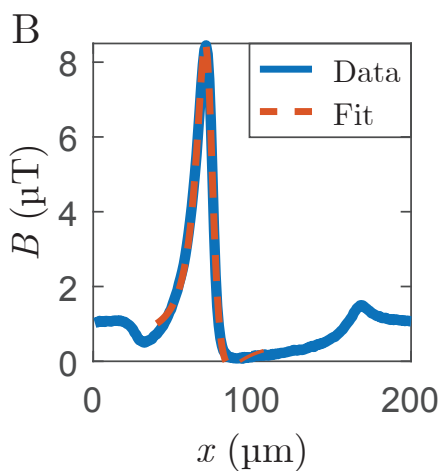

Figure 2.6: A) SSM image of an Abrikosov vortex in a Nb square. B) Line data shown in (A), with a fit using Equation 2.18.

which is higher than the $27^{\circ}$ angle that was used in the setup design. Part of this can be explained by looking at the cantilever that the sensor is mounted on: a flexible printed circuit board. Through repeated use or the significant difference between room temperature and measurement temperature $(4.2 \mathrm{~K})$, it is possible that this cantilever is deformed while scanning, which increases the angle.

In the second experiment to determine the scanning angle in our setup, we have made line scans of a magnetic field produced by a current in the $+y$-direction. Assuming for simplicity that the current line is infinitely long, the magnitude of the field is given by

$$
B=\frac{\mu_{0}}{2 \pi} \frac{I}{r^{2}}[z \sin \theta-x \cos \theta]
$$

Figure 2.7A and B show a schematic depiction and SSM image respectively of the current line when a current of $50 \mu \mathrm{A}$ is applied. Figure 2.7 shows the magnetic field data and a fit using the equation above. The data is taken from Figure 2.7B and averaged along the $y$-direction. The model fits the data well and allows us to extract the angle of the sensor, which is found to be approximately $45^{\circ}$. This is comparable to our findings in Figure 2.6 and is again significantly higher than $27^{\circ}$. This means that in our experiments the $x$-component of the magnetic field will play a considerable role. 

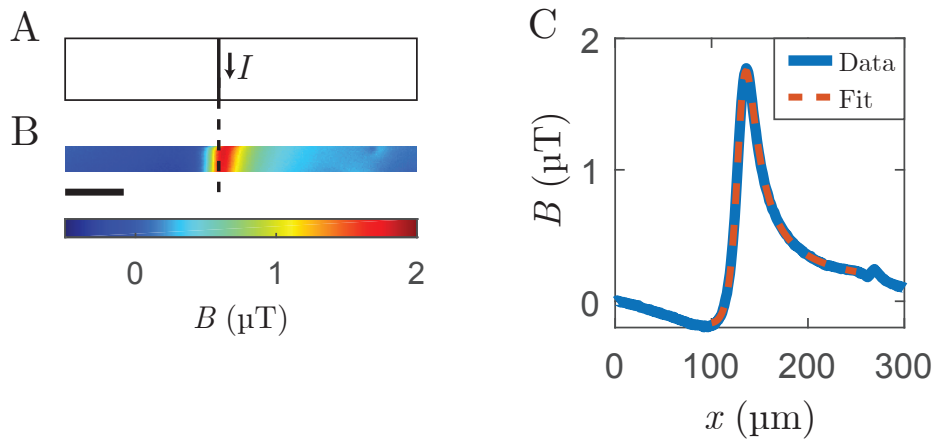

Figure 2.7: A) Schematic depiction of the scanning area with the current line and direction indicated. B) SSM image of the current line (indicated by the dashed line) with a current $I=50 \mu \mathrm{A}$. The scale bar indicates $50 \mu \mathrm{m}$. C) Averaged data from (B) with a fit using Equation 2.19.

\subsubsection{Magnetic shielding}

Our setup uses a $\mathrm{Nb}$ cylinder for additional magnetic shielding during scanning. When cooled, the $\mathrm{Nb}$ becomes superconducting and will expel most of the external magnetic field. To determine the effectiveness of this shield, we simulated it in COMSOL. The shield is modelled as a perfect magnetic insulator with a diameter of $6.5 \mathrm{~cm}$ in a $50 \mu \mathrm{T}$ background field (comparable to the Earth's magnetic field) along the $z$-axis. One factor to keep in mind is that as the helium in the cryostat evaporates, a smaller portion of the shield will be superconducting, reducing the shielding provided. We therefore determined the shielding effectiveness as a function of the cylinder height. Figure 2.8 shows the simulation results.

As we can see in Figure 2.8A, the shield repels almost all of the external magnetic field. Figure 2.8B shows that the background field is reduced by several orders of magnitude. In reality, there is a small hole at the bottom of the shield (approximately $1 \mathrm{~mm}$ in diameter) to allow for helium to enter the shield. When incorporating this hole into the model, we see that the shielding effectiveness is significantly reduced, and only a reduction of about 2 orders of magnitude is obtained.

We can also measure the noise spectrum and see how it changes when the shield is added. Figure $2.8 \mathrm{C}$ shows the noise as a function of frequency for both the shielded and unshielded case (data taken from Reference [10]). In both cases, we see a sharp drop above $1 \mathrm{kHz}$ due to a set of filters 
A

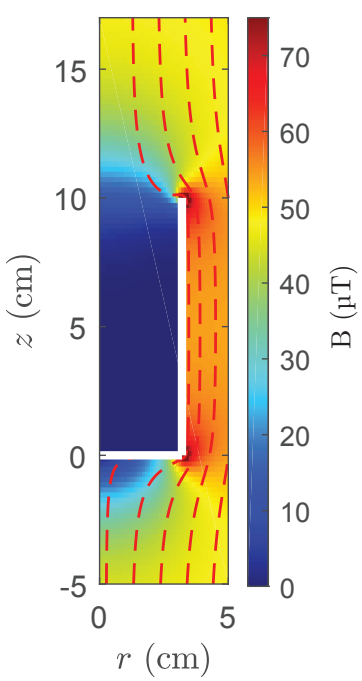

$\mathrm{B}$
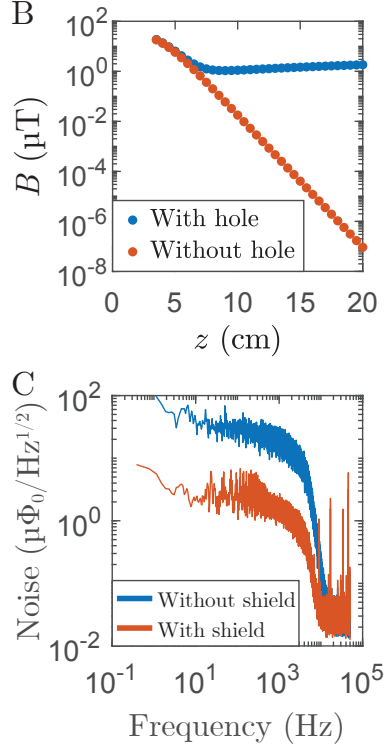

Figure 2.8: Simulation of the magnetic field around the shield used in the SSM setup. A) Magnetic field lines (red) around the magnetic shield (white). B) Magnetic field strength roughly at the location of the sensor, with and without hole in the bottom of the shield. C) Comparison between shielded and unshielded SSM setup noise spectrum (data from Reference [10]).

in the setup. Below roughly $10 \mathrm{~Hz}$, the system shows a $1 / f$ response, which is due to a variety of sources such as electron trapping and vortex movement [11-17]. Between $10 \mathrm{~Hz}$ and $1 \mathrm{kHz}$ is a region of constant noise level. In the unshielded case, this is roughly $20 \mu \Phi_{0} / \sqrt{\mathrm{Hz}}$, which equates a field resolution of approximately $100 \mathrm{nT}$. In the shielded case, we see a significant reduction across the whole spectrum. The region between $10 \mathrm{~Hz}$ and $1 \mathrm{kHz}$ is reduced by approximately an order of magnitude, giving us a noise level of $2.2 \mu \Phi_{0} / \sqrt{\mathrm{Hz}}$.

\subsection{Modelling of superconducting vortices}

Abrikosov vortices in Type II superconductors are one of the most commonly observed magnetic phenomena using SSM, including the first published SSM data by F.P. Rogers in 1983 [18]. Typical experiments involve the study of vortex behaviour in applied magnetic fields or at pinning 

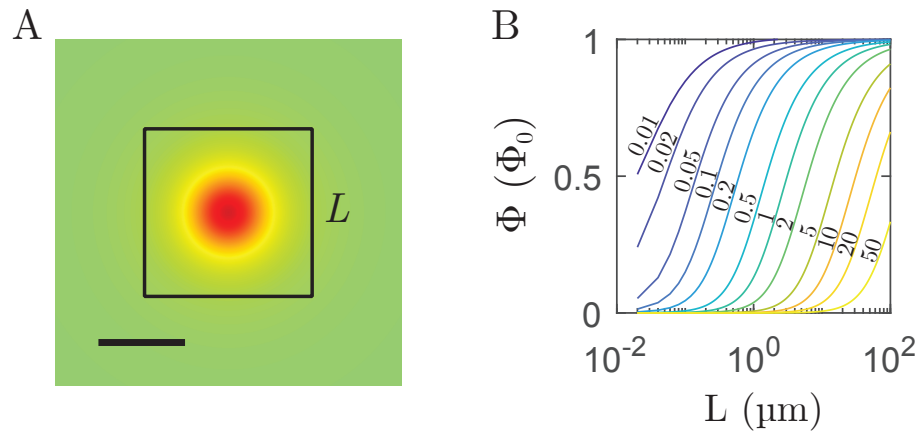

Figure 2.9: A) Simulated vortex with integration area of side length $L$. B) Total flux measured as function of $L$. The labels indicate different values of $h$ in $\mu \mathrm{m}$.

sites, such as material edges or grain boundaries (for a selection of works, see References [19-32]).

One type of measurement involves determining the total flux carried by a vortex. An Abrikosov vortex will always carry $1 \Phi_{0}$ of flux. However, if the total flux through the vortex deviates from $1 \Phi_{0}$ (known as a fractional vortex), it may be indicative of unconventional superconductivity in the system [33-37]. Therefore, being able to accurately determine the total flux coming from such an object is crucial for these experiments.

Figure 2.9A shows a simulated SSM image of an Abrikosov vortex modelled using Equation 2.17. In the ideal case, all flux coming from the vortex is imaged by the SSM sensor and the total flux will be $1 \Phi_{0}$. However, since the sensor will not be at $h=0$, the magnetic field coming from the vortex will have components in the $x$ - and $y$-directions to which the sensor is not sensitive. At $h=0$, a vortex should only have field lines along the $z$-direction. This is not captured by the monopole model, which predicts non-zero $x$ - and $y$-components even at $h=0$, but is reflected in more detailed descriptions of the vortex field $[38,39]$.

To determine the total flux, we integrate the data in the image over a certain area, indicated in Figure 2.9A as a square with side length $L$. Performing the integral algebraically, we get: 


$$
\begin{aligned}
\Phi & =\int_{-L / 2}^{L / 2} \int_{-L / 2}^{L / 2} \frac{\Phi_{0} h}{2 \pi r^{3}} d x d y, \\
\Phi & =\frac{2 \Phi_{0}}{\pi} \tan ^{-1}\left(\frac{L^{2}}{2 h \sqrt{4 h^{2}+2 L^{2}}}\right) .
\end{aligned}
$$

Ideally, one would take $L \rightarrow \infty$ to capture all flux. However, there are a few reasons not to do this. Obviously, an actual SSM image is of limited size. Furthermore, the magnetic field strength goes to zero rapidly for large $r$, so the contribution to the total flux becomes insignificant. Another issue is that there might be other magnetic objects near the vortex of interest, such as other vortices. These will have their own magnetic field that may overlap with that of the vortex, which can change the total flux if included in the integration area.

For this reason, we analysed the total flux as a function of $L$ for different values of $h$. The results of this are shown in Figure 2.9B. As expected, we see that the total flux approaches $1 \Phi_{0}$ as $L$ increases. Also, with increasing $h$, the integration area needs to be significantly larger to get close to $1 \Phi_{0}$. We find that to get within $10 \%$ error (i.e., $\Phi=0.9 \Phi_{0}$ ), $L>18 h$, and to get within $1 \%, L>180 \mathrm{~h}$. So for our own system, with an approximate height $h=5 \mu \mathrm{m}, L$ would need to be larger than $90 \mu \mathrm{m}$ to get an error less than $10 \%$.

\subsection{Modelling of ferromagnetic surfaces}

To extract more information out of measurement data, we can compare or fit data to calculations. In the case of ferromagnetic materials, the property of interest is often the magnetic moment. While a bulk measurement like vibrating sample magnetometry can give an average magnetic moment across the whole sample, there might be local variations. In this section, we will simulate ferromagnetic surfaces which we can compare to SSM data. This will allow us to estimate the magnetic moment of the material.

\subsubsection{Simulation method}

Ferromagnetic materials are usually made up of multiple magnetic domains with their own magnetic moment vectors. For our purpose, we are 


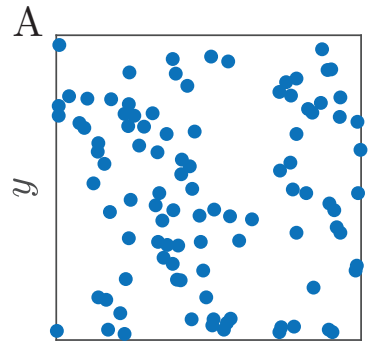

$x$

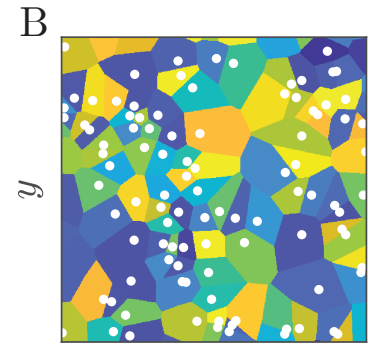

$x$

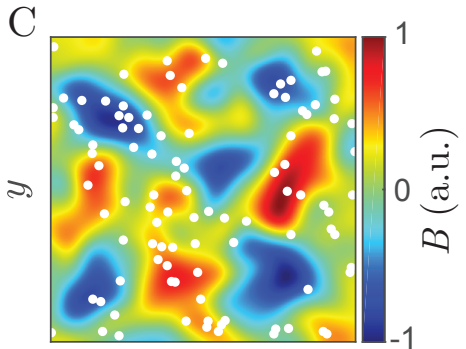

$x$

Figure 2.10: Steps in simulating a ferromagnetic surface. A) Placement of domain centres, also indicated in white in (B-C). B) Creation of domains around domain centres. Each coloured area is a single domain C) Calculated magnetic field.

not interested in the finer details of domain formation. We define a domain structure by randomly placing $N$ points on a given area, with $N$ being the number of domains (see Figure 2.10A), and then dividing the area into domains through a Voronoi diagram (Figure 2.10B). Each domain is assigned a direction for its magnetic moment.

The magnetic field is then calculated by assuming every pixel is a point dipole with the same magnetic moment. The magnitude of the magnetic moment is an input parameter; the direction is given by the domain that pixel belongs to. The field of each dipole is calculated and then summed to form the final image (Figure 2.10C). As an optional step, we can convolve the final image with a circle of diameter $d$ to simulate the effect of a finitely sized pickup loop.

All simulations are effectively done as a single, two-dimensional ferromagnetic layer. Most thin film systems under investigation, however, will consist of multiple layers. This can range from several monolayers to micrometers of material. Whether this model is justified depends on the thickness $t$ of the material that the simulation is being compared to. If $t \ll h$, then a magnetic moment on the bottom of the ferromagnetic layer will have a negligible difference in distance from the pickup loop compared to one on the surface. The final assumption is that moments align ferromagnetically along the $z$-axis.

For these simulations, we will use parameters that are typical for our SSM setup. We will simulate $50 \mu \mathrm{m} \times 50 \mu \mathrm{m}$ images as a balance between having a size comparable to typical SSM images, the detail of the simula- 

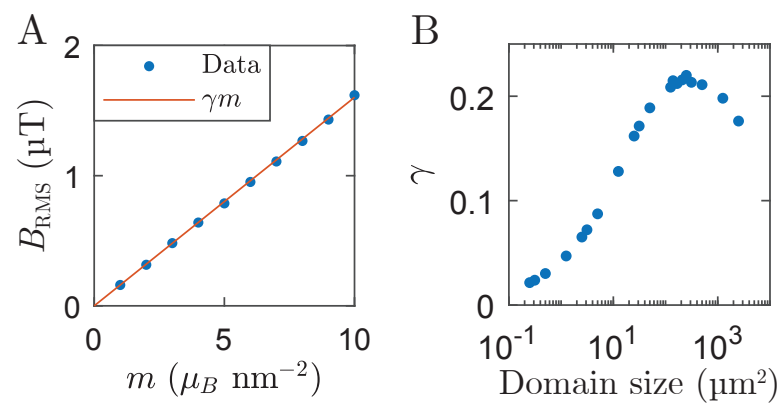

Figure 2.11: Relation between the magnetic moment $m$ and the resulting $B_{\mathrm{RMS}}$. A) $B_{\mathrm{RMS}}$ as a function of $m$, with a linear fit $B_{\mathrm{RMS}}=\gamma m$. B) $\gamma$ as a function of domain size.

tion and the simulation time.

We can model the influence of various parameters by varying them, generating multiple images and averaging over them. This is to reduce the influence of the fact that the domain structure is randomly generated. Often we will use the value $B_{\mathrm{RMS}}=\sqrt{\frac{1}{n} \sum_{i}^{n} B_{i}^{2}}$ to indicate the overall magnetic field strength of an image.

\subsubsection{Influence of the sample parameters}

To determine the magnetic moment from our SSM data, we can compare to the simulations as described above. Figure $2.11 \mathrm{~A}$ shows $B_{\mathrm{RMS}}$ as a function of $m$ for $h=5 \mu \mathrm{m}$ and $N=100$. As expected from the dipole equation, $B_{\mathrm{RMS}}$ increases linearly with increasing $m$. The slope of this curve $\gamma$ is the conversion factor we need to estimate a magnetic moment (per unit area) from SSM data. From Figure 2.11A, we find that $\gamma=$ $0.16 \mu \mathrm{T} \cdot \mathrm{nm}^{2} \cdot \mu_{\mathrm{B}}{ }^{-1}$.

The value of $\gamma$ depends on sample parameters such as $N$. Also, changing the direction between in-plane and out-of-plane will influence $\gamma$. Figure 2.11B shows how $\gamma$ depends on domain size $\left(\propto N^{-1}\right)$. We can see a local maximum in Figure 2.11B. For smaller domain sizes, the changes in magnetic field happen on a scale smaller than the resolution, meaning they get averaged out. The decrease at larger domain sizes is related to the fact that the moments are in-plane. This means that the magnetic field will only have an out-of-plane component near the domain boundaries. For larger domains, there will be fewer domain walls and therefore 

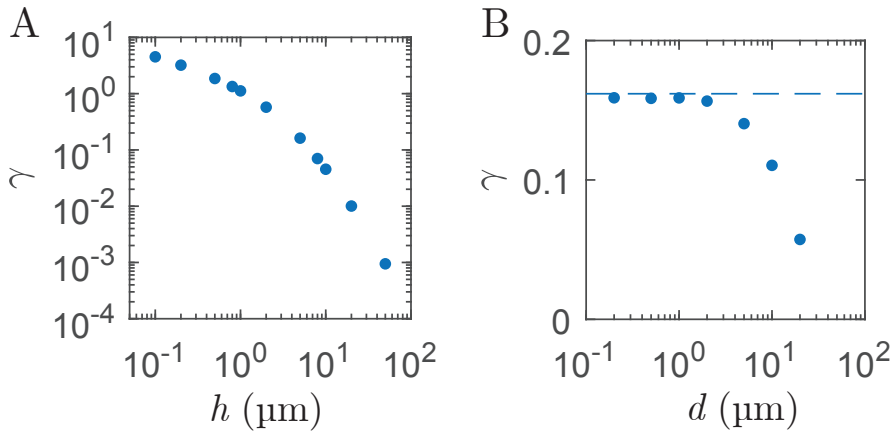

Figure 2.12: $\gamma$ as a function of A) scanning height $h$ and B) sensor diameter $d$. The dashed line in (B) indicates the unconvoluted case.

less magnetic field will be out-of-plane, leading to a reduction in $B_{\mathrm{RMS}}$. The location of the maximum is a balance between the domains being small enough to have many domain walls (where the out-of-plane magnetic field is strongest) and large enough to not be averaged out because of the limited spatial resolution.

\subsubsection{Influence of the measurement setup}

Going from sample to setup, two parameters that will influence the measurement data are the sensor height $h$ and sensor diameter $d$, which together determine the spatial resolution as discussed in Section 2.2.2. We will now have a more direct look at how these two parameters influence $B_{\text {RMS }}$. Figure 2.12A shows how $\gamma$ changes with increasing $h$ for $N=100$. We can see that it decreases rapidly as $h$ increases; this is in line with what we would expect from the dipole equation.

Figure 2.12B shows the influence of the finite sensor diameter, for $h=5 \mu \mathrm{m}$ and $N=100$. As we would expect, increasing $d$ reduces $\gamma$ due to the sensor averaging out the measurement data. For lower values of $d$, we can see that $\gamma$ does not vary much. Only when $d \approx h$ does $\gamma$ start to decrease. This is similar to Figure $2.4 \mathrm{C}$, where we observed different types of behaviour based on the relative values of $h$ and $d$.

As mentioned earlier, we model the sensor as a circular loop with a certain diameter. In a physical setup, the pickup loop is typically a more complicated shape, depending on the design and manufacturing process. However, the general trend observed in Figure 2.12B should still hold for 
increasing sensor area.

\subsection{Discussion and conclusion}

In this chapter we have looked at various aspects of the SSM setup and used simulations to investigate how different components influence the measurement data. After introducing the setup we looked at the spatial resolution. We defined it using a point-dipole, which is not only intuitive but also lends itself to a practical method of establishing the spatial resolution of a setup experimentally. We looked at how the spatial resolution changed as a function of two setup parameters, $h$ and $d$ (Figure 2.4C). We observed three regions: a height-dominated region, a diameter-dominated region and an intermediate region. In the first two, the spatial resolution is approximately equal to $h$ and $d$, respectively. In the intermediate region, the resolution can be calculated with Equation 2.16.

We then looked at the influence of having the sensor under a non-zero angle to the $x y$-plane. We determined experimentally that the angle in our setup is approximately $40^{\circ}$ by fitting SSM data to different models. Part of this is by design $\left(27^{\circ}\right)$, but the increase is likely due to the flexible cantilever the sensor is mounted on. Using a more rigid cantilever would solve this issue; however, the flexible cantilever will protect the sensor and sample from damaging one another. We also make use of the flexibility of the cantilever during approach: a set of strain gauges measures the force on the cantilever, allowing us to determine the point of contact and reduce $h$ as much as possible.

Our setup employs a $\mathrm{Nb}$ shield to reduce the influence of external magnetic fields. We modelled the shielding effectiveness in COMSOL and found the shield reduces the external field by several orders of magnitude (Figure 2.8). However, the shield has a small hole to improve liquid helium flow, and we found that this hole significantly impacts the effectiveness of the shield.

We then moved on to superconducting vortices and measuring their total flux. We looked at how the total measured flux changes as we vary the integration area as well as the height. We found that $L$ needs to be fairly large to get a good measure of the total flux ( $L>18 h$ for $10 \%$ error). This might complicate analysis where the measurement involves other nearby magnetic features.

Finally, we looked at simulated ferromagnetic surfaces. We established 
a factor $\gamma$ that relates $B_{\mathrm{RMS}}$ to the average magnetic moment. This allows us to extract the magnetic moment per unit area from SSM imagery. While this value will be a average value across the imaged area (in the same way that techniques like vibrating sample magnetometry yield a bulk value), it can still provide reasonably local data.

We studied how $\gamma$ changed as we changed various parameters. We looked at the magnetic moment $m$ and number of domains $N$ (Figure 2.11), as well as the sensor height $h$ and diameter $d$ (Figure 2.12). As a function of $N$, we noticed a local maximum in $\gamma$, which we interpreted as a balance between the number of domain walls and averaging due to the spatial resolution. For $h$ and $d$, we noticed similar behaviour to Figure $2.4 \mathrm{C}$, where we observed different regions depending on the relative values of $h$ and $d$.

In order to use $\gamma$ to extract $m$, the parameters $N, h$ and $d$ need to be known for the experiment. Determining $N$ (i.e. the domain size) is the hardest of the three, since it will require either an SSM setup with very high spatial resolution or a different scanning probe method, such as magnetic force microscopy. Similarly, $h$ and $d$ may also be difficult to assess with high accuracy. This method, therefore, will not likely give a very accurate value for $m$ but serves more as a rough estimate to compare with other experiments.

The influence of the sensor is a convolution of the measurement data with the sensor shape. This convolution can be reversed by establishing the function that describes the sensor shape (sometimes described as the point-spread function). However, the presence of noise complicates this mathematical inversion. In addition, deconvolution is not necessarily unique, meaning the recovered data might not be representative of the actual magnetic features that were imaged. Some research into deconvolution applied to SSM imaging has been done [40,41], but the field could benefit from more rigorous mathematical models. 


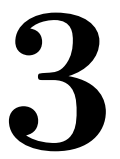

\section{Scanning SQUID microscopy and magnetometry on low- $T_{c} /$ high $-T_{c}$ hybrid junctions}

This chapter will present results from a collaboration with the Forschungszentrum Jülich on low- $T_{c} /$ high- $T_{c}$ junctions and $\pi$-loops. The goal is to measure and manipulate fractional flux quanta, with a focus on stabilising higher flux states beyond the $\pm \frac{1}{2} \Phi_{0}$ ground state.

I would like to thank Prof. Dr. Michael Faley for the collaboration and the fabrication of the samples, and Dimas Christoforus Satrya, M.Sc. and Bart Folkers, B.Sc. for helping me with the measurements and analysis of the data. Sections 3.4 and 3.5 of this chapter have been published as M.I. Faley et al., $\pi$-Loops With ds Josephson Junctions, IEEE Transactions on Applied Superconductivity, 2019. Section 3.6 is (at time of writing) being prepared for publication. 


\subsection{Introduction}

In 1986 Bednorz and Müller stumbled upon the first high-critical-temperature (high- $T_{c}$ ) superconductor, $\mathrm{Ba}_{\mathrm{x}} \mathrm{La}_{5-\mathrm{x}} \mathrm{CuO}_{5(3-\mathrm{y})}$ [42]. In the next few years, the record $T_{c}$ shot up from the old record of $23 \mathrm{~K}$ of $\mathrm{Nb}_{3}$ Ge to well over $100 \mathrm{~K}$ in Hg-based cuprates, and room-temperature superconductivity was believed to be within reach. Meanwhile, theoreticians were painstakingly trying to explain these new superconducting materials that seemingly did not obey the previously established theories of Bardeen, Schrieffer and Cooper.

While the current record holder for $T_{c}, \mathrm{LaH}_{10}$ at $250 \mathrm{~K}$ under extreme pressures [43], is still a ways away from ambient conditions, and a definitive microscopic description for high- $T_{c}$ superconductivity remains to be agreed upon (for a discussion, see Reference [44]), technology has eagerly made use of these materials: Replacement computing technology such as rapid-single-flux-quantum (RSFQ) structures, $\mathrm{RF}$ and microwave filtering systems and SQUIDs, but also larger-scale applications such as for electromagnets in NMR and MRI systems, particle accelerators or nuclear fusion systems.

In this chapter, we will look at a specific kind of SQUID based on combinations of high- $T_{c}$ and BCS low- $T_{c}$ superconductors. By exploiting the unusual symmetry of the order parameter in superconducting cuprates, spontaneous flux can be generated in these so-called $\pi$-loops. Interestingly, this spontaneous flux has a value of $\frac{1}{2} \Phi_{0}$, where $\Phi_{0}=2.0678 \times 10^{-15} \mathrm{~T} \cdot \mathrm{m}^{2}$ is the magnetic flux quantum. This state is naturally bistable (both $+\frac{1}{2} \Phi_{0}$ and $-\frac{1}{2} \Phi_{0}$ are valid ground state solutions) and has already been shown to be useful in RSFQ systems, eliminating the need for bias currents to create a degenerate ground state [45].

Here we will attempt to access higher flux states with use of a onchip magnetic bias. By running a bias current through a strip line, a local magnetic field is produced that can be used to influence these $\pi$-loop structures. We will investigate the ability to induce higher flux states and the stability of them, and we will look at how the stability of the different states changes when using different superconducting materials. The ability to stabilise and manipulate different states could be an important step towards new computing concepts such as quantum annealing computing [46-48]. 
A

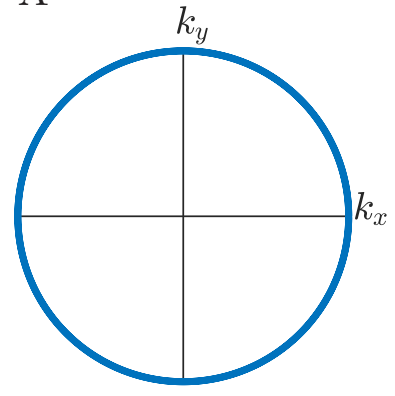

B

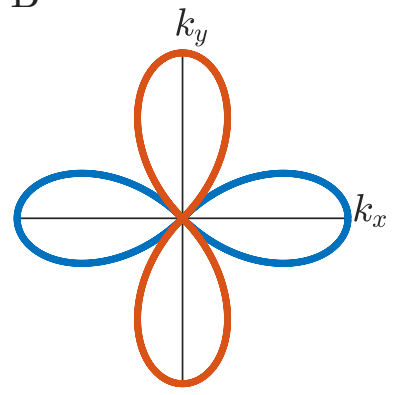

C

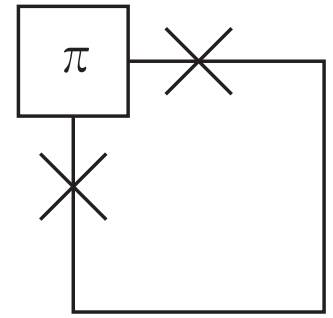

Figure 3.1: Symmetry and phase of the order parameter in A) $s$-wave and B) $d$-wave superconductors. Regions of different colour in (B) have a $\pi$ phase-shift between them. C) Circuit diagram of a $\pi$-loop.

\subsection{Spontaneous half flux quanta in $\pi$-loops}

When we looked at flux quantisation in Chapter 2, we expanded it to include phase drops across any junctions in the loop. However, we neglected a secondary phase component: the phase of the superconducting order parameter.

Typically, superconducting circuitry is made out of Nb, a BCS superconductor. The pairing symmetry and phase of such a superconductor is displayed in Figure 3.1A. As we can see, the pairing symmetry is isotropic and the phase is constant. This is often referred to as an $s$-wave superconductor, due to the identical symmetry of the $s$-orbital in atomic physics. This allowed us to ignore the contribution to the fluxoid quantisation condition.

Things change when we include components made out of superconductors that have a different pairing symmetry. In the 1990's, it was discovered that high- $T_{c}$ superconductors such as $\mathrm{YBa}_{2} \mathrm{Cu}_{3} \mathrm{O}_{7}$ have a $d$ wave pairing symmetry (akin to the symmetry of $d$-orbitals, see Figure 3.1B) $[33,49,50]$. This has two consequences. First, the gap size is no longer isotropic, but has a strong angular dependence. Second, there is a $\pi$ phase shift between the $k_{x}$ and $k_{y}$ directions.

Because of this, in a simple junction like Figure 3.1C, the YBCO component will induce a $\pi$ phase shift when going around the loop. This 
means that the fluxoid quantisation condition for such a loop becomes

$$
n=\frac{1}{2 \pi}\left(\varphi_{1}+\varphi_{2}+\pi\right)+\frac{\Phi}{\Phi_{0}},
$$

where $\varphi_{1}$ and $\varphi_{2}$ are the phases associated with the Nb-YBCO contacts, which behave as Josephson junctions.

The ground state of this system is no longer the trivial solution of zero phases and zero flux. The extra $\pi$ phase shift has to be compensated for by altering the phases and the total flux. The exact solution depends on the details of the loop (which will be discussed in the next section), but in the limit of the inductance $L$ going to infinity, $\varphi_{1}$ and $\varphi_{2}$ will become negligible and we get

$$
\Phi=\frac{1}{2} \Phi_{0}
$$

So even in the absence of an external field, a loop containing an element with a $\pi$ phase shift (known as a $\pi$-loop) will generate a spontaneous flux equal to $\frac{1}{2} \Phi_{0}$. This ground state is degenerate, since both flux directions (up and down) are valid solutions.

We can also have a look at the free energy $U$ of a $\pi$-loop. In literature, these systems are often modelled as a circuit with a single Josephson junction and a $\pi$-shift element [51]. In such a model, the energy can be written as:

$$
U_{\text {single }}(\Phi)=\frac{\left(\Phi-\Phi_{e}\right)^{2}}{2 L}+2 E_{J} \cos \left(\frac{2 \pi \Phi}{\Phi_{0}}+\pi\right) .
$$

However, a $\pi$-loop will consist of two junctions, formed by the two points where the high- $T_{c}$ and low- $T_{c}$ superconductors connect, as well as a $\pi$-shift element. In such a model, the energy is written as

$$
U=\frac{1}{2} L I^{2}-E_{j}\left(\cos \varphi_{1}+\cos \varphi_{2}\right),
$$

where $I$ is the current in the loop.

This equation can be rewritten using 3.1 and by noting that the total flux $\Phi$ is the sum of the external flux $\Phi_{e}$ and the flux due to the screening current $L I$ :

$$
U_{\text {double }}=\frac{\left(\Phi-\Phi_{e}\right)^{2}}{2 L}-E_{J} \sqrt{2-2 \cos \left(\frac{2 \pi \Phi}{\Phi_{0}}\right)} .
$$




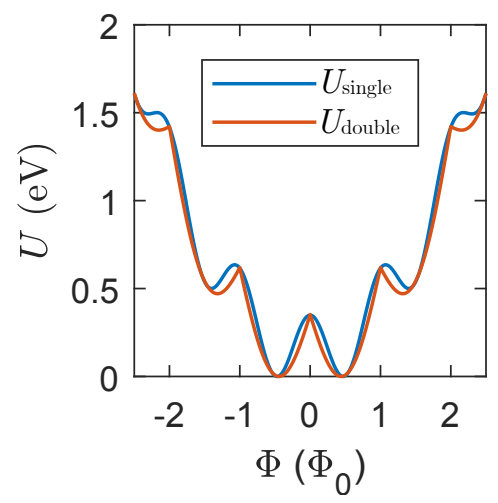

Figure 3.2: Comparison between the single-junction and double-junction models for the energy $U$ of a $\pi$-loop as function of the total flux $\Phi$.

Figure 3.2a shows these two functions. Since the system will try to minimise its energy, the actual value of $U(\Phi)$ at any point $\Phi$ will be whichever of the two functions has lower energy. Figure 3.2 shows a comparison between equations 3.3 and 3.5. We can see that the single-junction model has slightly shallower minima near the $\Phi=\left(n+\frac{1}{2}\right) \Phi_{0}$ points, and that the minima are at higher (absolute) values of $\Phi$.

\subsection{Influence of the loop inductance}

As mentioned in the previous section, one factor that needs consideration when imaging fractional vortices is the loop inductance. When looking at the fluxoid quantisation equation:

$$
n=\frac{1}{2 \pi}\left(\varphi_{1}+\varphi_{2}+\pi\right)+\frac{\Phi}{\Phi_{0}},
$$

we can expand the expression for the total flux $\Phi$ :

$$
\Phi=\Phi_{e}+L I
$$

where $\Phi_{e}$ is any external flux, and $L I$ is the flux generated by the loop. Since the current in the loop has to be the same everywhere, and assuming $I_{c 1}=I_{c 2}=I_{c}$, we can state:

$$
\begin{array}{r}
I=I_{c} \sin \left(\varphi_{1}\right)=I_{c} \sin \left(\varphi_{2}\right), \\
\varphi_{1}=\varphi_{2} \text { or } \varphi_{1}=\pi-\varphi_{2}
\end{array}
$$



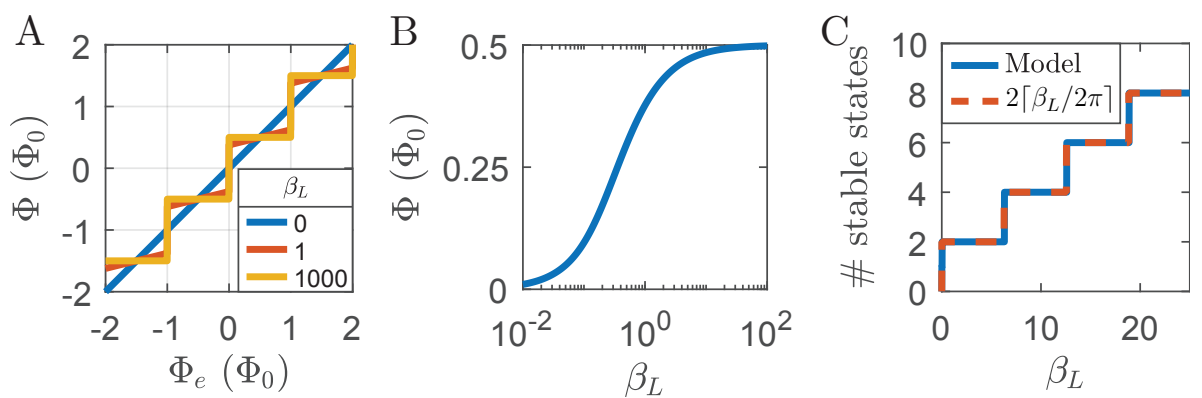

Figure 3.3: Influence of the loop inductance on the total flux. A) Total flux as a function of external flux for different values of the inductance. B) Total flux at zero external flux as function of the inductance. C) Number of stable states in a $\pi$-loop as function of $\beta_{L}$.

Often in such loops, the inductance $L$ is expressed as a ratio $\beta_{L}$

$$
\beta_{L}=\frac{2 \pi I_{c} L}{\Phi_{0}}
$$

Combining the above, we find

$$
\Phi=\frac{\Phi_{e}}{\Phi_{0}}+\frac{1}{2 \pi} \beta_{L} \sin \left(\varphi_{1}\right) .
$$

We can see that the total flux (i.e., the measured flux using SSM) depends on the inductance of the structure. This allows us to rewrite the fluxoid quantisation condition

$$
n=\frac{1}{2 \pi}\left(2 \varphi_{1}+\pi\right)+\frac{\Phi_{e}}{\Phi_{0}}+\frac{1}{2 \pi} \beta_{L} \sin \left(\varphi_{1}\right) .
$$

We can now solve this equation to see what $\Phi$ would be for different values of $\Phi_{e}$ and $\beta_{L}$. Figure 3.3a shows the total flux as a function of the applied flux for several values of $\beta_{L}$. It shows that for high inductance loops, the flux does indeed become $\left(n+\frac{1}{2}\right) \Phi_{0}$. But at low inductance, depending on the applied flux, the total flux can deviate significantly from $\left(n+\frac{1}{2}\right) \Phi_{0}{ }^{1}$.

To see this more clearly, Figure 3.3B shows the total flux at $\Phi_{e}=0$ for varying $\beta_{L}$. Again we see that at high $\beta_{L}$, the flux approaches $\frac{1}{2} \Phi_{0}$ as

\footnotetext{
${ }^{1}$ For ease of reading, we will refer to different flux states as $\left(n+\frac{1}{2}\right) \Phi_{0}$ throughout this chapter, even though the actual flux values will deviate based on $\beta_{L}$. However, $\beta_{L}$ of our devices is high enough that the flux will be close to $\left(n+\frac{1}{2}\right) \Phi_{0}$.
} 
expected. But for $\beta_{L} \leq 100, \Phi$ starts to deviate from $\frac{1}{2} \Phi_{0}$ significantly. Therefore, finding lower-than-expected values for $\Phi$ during analysis can be simply a feature of the system, rather than an error in measurement. Through careful modelling of the system beforehand, the inductance can be estimated and with that, the expected measured flux.

Coming back to the free energy $U$ that we derived in Section 3.2, we can look at the influence of the inductance on the loop properties. Looking at Equation 3.5, we see that the product $I_{c}$ and $L$ (and therefore $\beta_{L}$ ) determine the barrier height and with that, the number of available stable states. Figure 3.3C shows the number of states that are stable without any external bias, i.e., the number of local minima. We find that there are always an even number of stable states, and the amount increases with increasing $\beta_{L}$ as expected. The number of stable states seems to closely follow $2\left\lceil\beta_{L} / 2 \pi\right\rceil$, also shown in Figure 3.3C.

\subsection{Design and fabrication of $\pi$-loops}

Samples for these experiments were fabricated at the Peter Grünberg Institute of Forschungszentrum Jülich. As outlined previously, the loops are a combination of low- $T_{c}$ and high- $T_{c}$ superconductors, in order to achieve spontaneous flux generation.

The junctions are fabricated in a 'ramp-type' style (Figures $3.4 \mathrm{~A}$ and B). A classic planar junction would require the current to flow along the $c$-axis of the system. Since superconductivity in YBCO is confined to the $a b$-plane and significantly suppressed in the $c$-direction, such a planar structure would have a very low critical current. For this reason, ramptype junctions have been shown to be a good solution. A thin Au layer is added between the $\mathrm{Nb}$ and YBCO to improve barrier transparency [52].

In previous experiments, the junctions were grown on $\mathrm{SrTiO}_{3}$ substrates. However, thicker YBCO films (beyond $\sim 300 \mathrm{~nm}$ ) will start to crack due to the lattice mismatch with the substrate. To improve the quality of the YBCO film, the samples are fabricated on $\mathrm{MgO}$ substrates covered with a YBCO-STO buffer layer [53]. $500 \mathrm{~nm}$ YBCO followed by $160 \mathrm{~nm}$ STO are then sputter-deposited at high oxygen pressure. After application of a photolithographic mask, the YBCO squares that form the $\pi$-shift elements of the loops are defined by Ar ion milling. The milling is done at a $60^{\circ}$ angle with the surface normal to create the ramps. After removal of the photoresist, a $6 \mathrm{~nm}$ thick YBCO recovery layer, a $6 \mathrm{~nm} \mathrm{Au}$ 
A

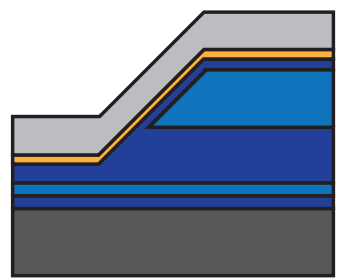

$\square \mathrm{MgO} \quad \square \mathrm{Au}$
$\square \mathrm{YBCO \quad} \quad \mathrm{Nb}$
$\square \mathrm{STO}$
B

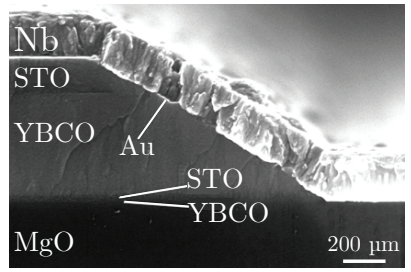

C

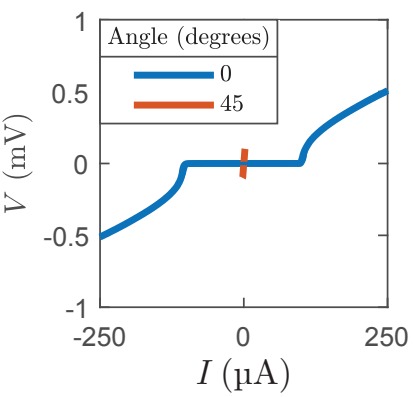

Figure 3.4: A) Schematic depiction of a Nb-YBCO ramp junction. B) Scanning electron microscopy image of a Nb-YBCO ramp junction. C) Critical current measurement of Nb-YBCO ramp junctions at different angles with the YBCO crystal axes.

interlayer and finally the $200 \mathrm{~nm} \mathrm{Nb}$ layer are grown using DC magnetron sputtering in a 0.01 mbar Ar atmosphere. The $\mathrm{Nb}$ is then structured using another photolithographic mask and a second Ar ion milling step.

Figure 3.4C shows critical current measurements on the junctions of $3 \mu \mathrm{m}$ width for two orientations: one junction along the YBCO crystal axes, and one at a $45^{\circ}$ angle (the nodal direction of $d_{x^{2}-y^{2}}$ symmetry). At $0^{\circ}$, we find that $I_{c}=100 \mu \mathrm{A}$ and an $I_{c} R_{n}$ product of $200 \mu \mathrm{V}$. Along the nodal direction, the critical current is reduced by over two orders of magnitude, matching our expectations of suppressed superconductivity in this direction.

\subsection{Measurements on Nb-based hybrid junctions}

\subsubsection{Square $\pi$-loop array}

To verify whether the $\pi$-loops do indeed exhibit the $\frac{1}{2} \Phi_{0}$ ground state, we imaged the $\pi$-loop array using SSM. A $500 \mu \mathrm{m} \times 500 \mu \mathrm{m}$ image is shown in Figure 3.5B. The image shows the square lattice and the individual loops are clearly visible. The field at every site has the same profile, meaning that the flux through every loop is the same. We can also recognise the rough shape of the physical structure of the $\pi$-loop in the magnetic field (see insets in Figure 3.5). By isolating a single loop (see inset of Figure 

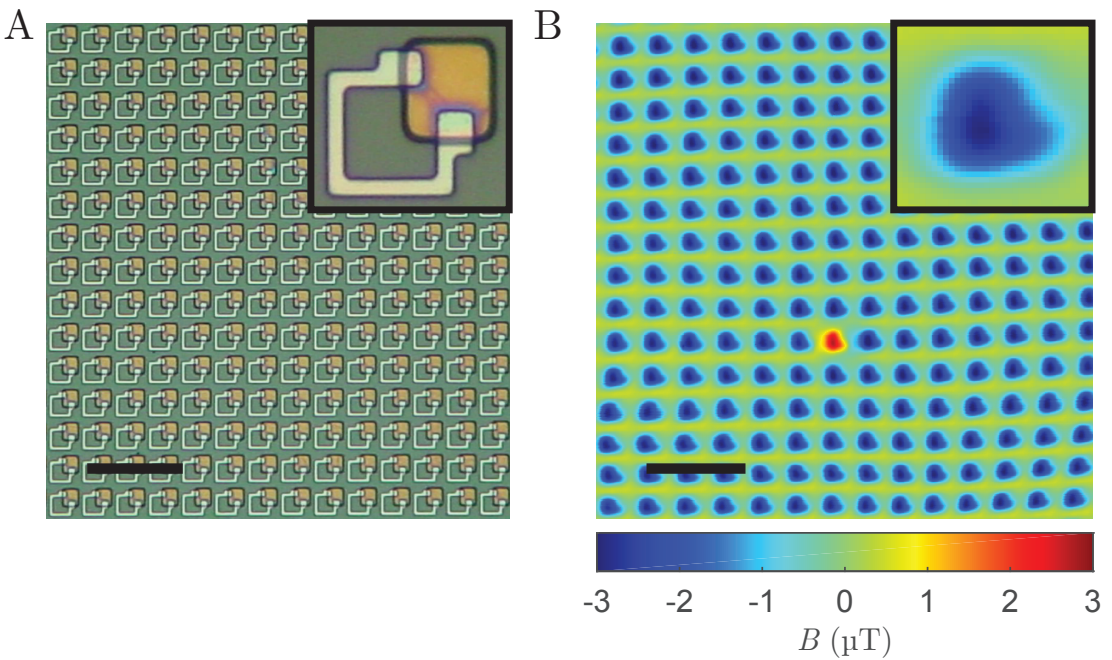

Figure 3.5: A) Optical image and B) SSM image of an array of Nb-YBCO $\pi$-loops. Scale bars indicate $100 \mu \mathrm{m}$. Insets show a zoom on a single $\pi$-loop.

3.5B), we can calculate this flux by integrating over the area. We find that the total flux is approximately $0.46 \Phi_{0}$.

As explained above, a loop will only produce $0.5 \Phi_{0}$ in the large inductance limit. Using the 3D-MLSI software package, we can model the inductance for these loops, which turns out to be $23 \mathrm{pH}$, yielding $\beta_{L}=14$. From Figure 3.3B, this gives us an estimated flux of $0.44 \Phi_{0}$. So our measured value is actually higher than what we would expect based on simulations. This could be partly due to an error in the calibration of the SQUID area (see Chapter 2). Another option could be that due to the close proximity of other loops, the overlapping fields will influence the total measured flux. Other possibilities include not properly choosing the integration area (as discussed in Chapter 2) or an error in the simulated value for $L$.

We also see that the magnetic field in most loops points along the same direction. Since vortices couple antiferromagnetically, if the coupling were strong enough, we would expect a checkerboard pattern to emerge. Alternatively, if the coupling is too weak, each vortex would have a random orientation. The ferromagnetic pattern observed in the array likely indicates a small background field in the same direction, leading to a preferential orientation for the vortices. While the measurement was per- 
A

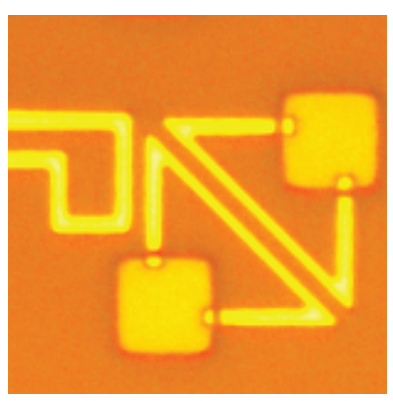

B

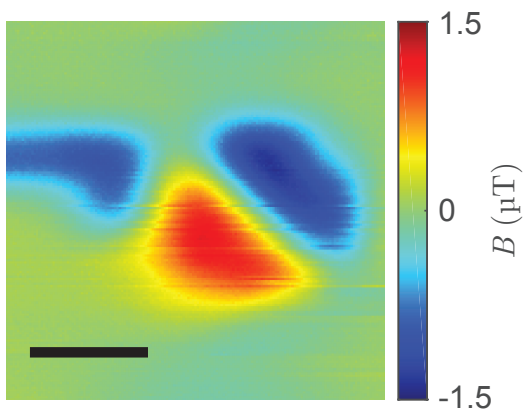

Figure 3.6: A) Optical and B) SSM image of a pair of triangular $\pi$-loops and a nearby bias line. The scale bar indicates $50 \mu \mathrm{m}$.

formed in a shielded setup, only a small magnetic bias would be enough to orient the array along the same direction while cooling.

\subsubsection{Paired triangular $\pi$-loops with external bias field}

The next set of measurements focuses on paired triangular loops. For this, a new sample was fabricated with such loops, as well as Nb current lines to allow for magnetic field biasing. Figure 3.6A shows an optical image of such a pair, along with the nearby bias line. Figure 3.6B shows an SSM image of the same area. The flux through the rings has a triangular profile, matching their physical geometry. In addition, the flux through both rings is equal and opposite, and again comes to a total of $\sim \frac{1}{2} \Phi_{0}$ each. The bias line is made visible by passing a small current through it.

We use the bias line to apply a magnetic field to the loops. If this magnetic field is strong enough, the vortex direction should flip to match the applied field direction. For this experiment, the SQUID sensor was placed above the loop of a pair closer to the bias line. Figure 3.7A shows several sweeps of $I_{\text {bias }}$ between $\pm 1.5 \mathrm{~mA}$ and the recorded SQUID voltage. We can see large jumps in the voltage at both positive and negative bias, indicating a large, instantaneous change in the magnetic field in the loop. This could then be the $\pi$-loop switching between different flux states.

To check this, we took SSM images to visualize the configuration of the loops at specific points in the sweep. The upper images in Figure 3.7D were taken at points 1 and 2 in Figure 3.7A. As expected, we see that one of the $\pi$-loops has switched between a positive and negative flux state. 

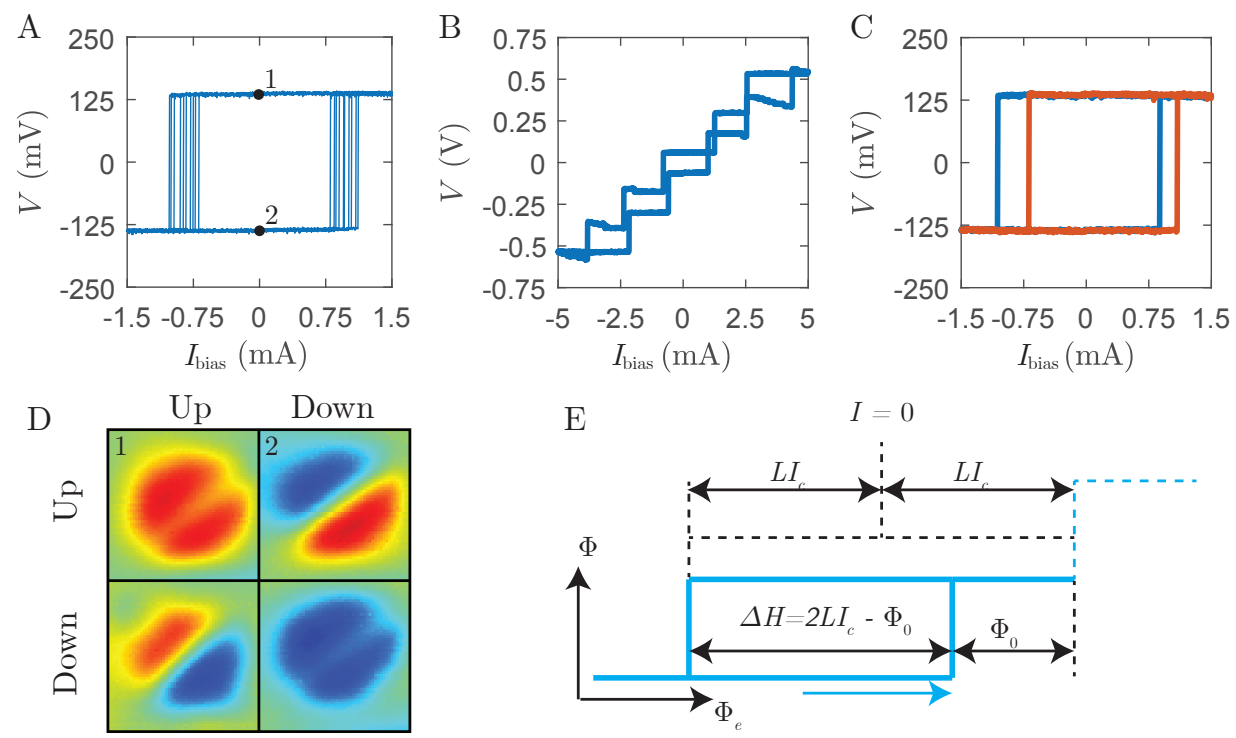

Figure 3.7: Flux $\Phi$ through a triangular junction as a function of bias current $I_{\text {bias }}$. A) Multiple field sweeps with the second loop in a fixed state. B) Hysteresis measurement over a large current range. C) Comparison between two sweeps with the second loop in different states. D) SSM images of the four different states available at zero applied field. E) Diagram illustrating how different parameters can be extracted from a hysteresis loop.

This confirms that we can use the bias line to switch between different flux states.

In Figure 3.7B we have increased the sweep range to $\pm 5 \mathrm{~mA}$. We clearly see additional jumps in the voltage at higher biases, indicating higher flux states. Each jump has the same magnitude, which means that the same amount of flux is added each time. However, we also find that these higher states are not stable at zero bias (the loop falls back to the $\pm \frac{1}{2} \Phi_{0}$ state before reaching $\left.I_{\text {bias }}=0\right)$.

We can also see smaller voltage jumps in Figure 3.7B. These originate from other nearby loops switching due to the applied field. At $I_{\text {bias }} \approx$ $\pm 2.2 \mathrm{~mA}$, the second loop in the pair of triangles under investigation is switched. This is again confirmed by taking SSM images, as shown in the lower images in 3.7D.

To analyse the data further, we can convert the applied current into an applied flux $\Phi_{e}$. We can do this by noting that the free energy $U(\Phi)$ is 
periodic in $\Phi_{0}$. Therefore, by applying larger bias current and switching to higher states, each successive switching point in Figure 3.7B must be $\Phi_{0}$ away from the last. This allows us to define a parameter $\eta$, which is the conversion factor between applied current and applied flux: $\Phi_{e}=\eta I_{\text {bias }}$. By averaging over 100 sweeps, we find that $\eta=1.63 \Phi_{0} \cdot \mathrm{mA}^{-1}$.

To confirm the influence of the upper loop, we can apply a larger bias field to change its orientation, which should result in a coupling bias in the other direction. Figure 3.7C shows two hysteresis loops, one for each direction of the upper loop. We see a clear change of the coupling bias, confirming that the upper loop does influence the energy landscape of the lower loop. By converting the horizontal shift from current to flux using $\eta$, we find that the secondary loop couples $0.031 \Phi_{0}$ into the primary.

We can confirm this by simulating the loops. We do this by simulating a current loop shaped like the upper triangle and calculating the flux through the area that would be taken up by the lower triangle (see Figure 3.8). By numerically integrating the Biot-Savart law over the area $A$, we obtain the flux through the triangle:

$$
\Phi=\int_{A}\left[\frac{\mu_{0} I}{4 \pi} \oint_{C} \frac{\delta \vec{\imath} \times \vec{r}}{r^{3}}\right] d A,
$$

where $I$ is the current through the upper triangle, $\vec{r}$ the distance from the current source and $C$ the current path. We can calculate $I$ by solving the fluxoid quantisation condition, giving us the phase $\varphi$, which yields the current through $I=I_{c} \sin (\varphi)$.

By simulating a triangle in the 3D-MLSI software package, we ob-

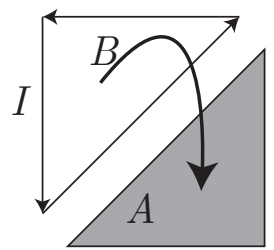

Figure 3.8: Schematic depiction of the model used to calculate the flux through one loop in a pair originating from the other. tain an inductance of $36 \mathrm{pH}$. From the measurements in Figure 3.4 we know that $I_{c}=100 \mu \mathrm{A}$. This gives $I=24 \mu \mathrm{A}$. We then obtain a flux through the lower triangle of $0.020 \Phi_{0}$. This closely matches the value of $0.031 \Phi_{0}$ we derived from our measurements.

Next, we see that the magnitude of each flux jump is the same as the first, and the separation along the $x$-axis is also equal. Both of these indicate that each transition point adds an additional $\Phi_{0}$ of flux, just as 

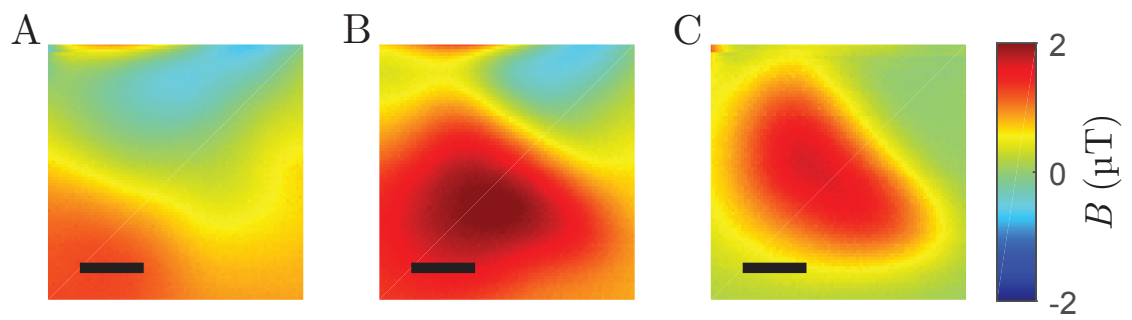

Figure 3.9: Image subtraction to visualise a flux jump. A-B) SSM images of A) before and B) after a flux jump. C) Difference between (A) and (B). Scale bars indicate $20 \mu \mathrm{m}$.

predicted. To confirm this, we have made images right before and after a jump. By subtracting these two images from one another, the result will be the flux added/removed during the transition. Figure 3.9 shows the two images from before and after the transition, and the difference between the two. Upon integrating over Figure 3.9C, we find the flux to be approximately $0.92 \Phi_{0}$, showing that we indeed add or remove one $\Phi_{0}$ at a switching point.

We can attempt to extract $\beta_{L}$ from the hysteresis loop, since this value determines the barrier height. Looking at the schematic loop in Figure 3.7E, we can note a few things. The distance between two successive jumps is $\Phi_{0}$, as noted before. Next, we can deduce that the total flux range over which any specific state is stable is equal to $2 I_{c} L$ : The current exactly halfway in the stable range must be zero, because at this point $\Phi_{e}=\Phi$. Since switching occurs when the critical current is reached, the switching points must be $I_{c} L$ away from this centre point. Therefore, the full range must be $2 I_{c} L$. Finally, we can see that the width of a single hysteresis loop, denoted $\Delta H$, must then equal $2 I_{c} L-\Phi_{0}$.

Going back to Figure 3.7A, we can extract $\Delta H / \eta=1.8 \mathrm{~mA}$, which gives $\beta_{L}=6.4$. We can extract the same information from the larger sweep in Figure 3.7B. Here we find $\beta_{L}=6.1$. Based on the measured $I_{c}=100 \mu \mathrm{A}$ and the simulated inductance $L=36 \mathrm{pH}$, we would expect $\beta_{L}=11$, a factor 1.7 difference.

Based on Figure 3.3C, the values we found for $I_{c} L$ would suggest that the triangular Nb-based loop sits close to the transition between 2 and 4 stable states $\left(\beta_{L} / 2 \pi \approx 1\right)$. That would mean that the transitions to the $\pm \frac{3}{2} \Phi_{0}$ states should lie very close to $I=0$. However, this does not seem 
to be the case, judging from Figure 3.7B.

\subsection{Measurements on MoRe-based hybrid junc- tions}

In an attempt to stabilize the higher flux states we need to increase $\beta_{L}$, which is the dominant factor in determining the energy barrier height (see Equation 3.5 and Figure 3.3). Since $\beta_{L} \propto I_{c} L$, we can achieve this by increasing the critical current and/or the loop inductance.

The critical current can be increased by either using more superconducting material, or by changing to a different superconductor. While making a thicker superconducting layer is relatively easy, it will also change the loop inductance, which makes it difficult to change the parameters independently. Using a different superconductor does allow for this, since the same loop geometry will have the same inductance (neglecting kinetic inductance) for different materials, but different critical current. The inductance can then independently be changed by altering the loop design.

\subsubsection{MoRe-YBCO junctions}

In order to achieve a higher $I_{c}$ and $\beta_{L}$, we substituted $\mathrm{Nb}$ for MoRe. MoRe is a BCS superconductor with a $T_{c}$ up to $13 \mathrm{~K}$ [54-56]. We measured our MoRe to have $T_{c}=9 \mathrm{~K}$ (Figure 3.10A). The MoRe-YBCO hybrid junctions were fabricated in the same way as the $\mathrm{Nb}-\mathrm{YBCO}$ junctions described in Section 3.4.

Figure 3.10B shows the $I-V$ characteristics of these junctions, which have a width of $3 \mu \mathrm{m}$. We can see that $I_{c}=165 \mu \mathrm{A}$ along the YBCO crystal axes. For a junction making an angle of $45^{\circ}$ with the YBCO crystal axes (corresponding to the nodal direction of the $d_{x^{2}-y^{2}}$-wave symmetry), the critical current is two orders of magnitude smaller. In Figure 3.10C we compare the $\mathrm{Nb}$ and MoRe based junctions. The MoRe-YBCO junctions have an $I_{c}$ that is approximately $65 \%$ larger.

We also find that the MoRe-YBCO junctions have a significant hysteresis, with a retrapping current $I_{r}=85 \mu \mathrm{A}$. This is due to the higher $I_{c} R_{n}$ product, $800 \mu \mathrm{V}$, which means the Stewart-McCumber parameter $\beta_{C}=\frac{2 \pi I_{c} R_{n}^{2} C}{\Phi_{0}}>1$ and the junction is underdamped. 

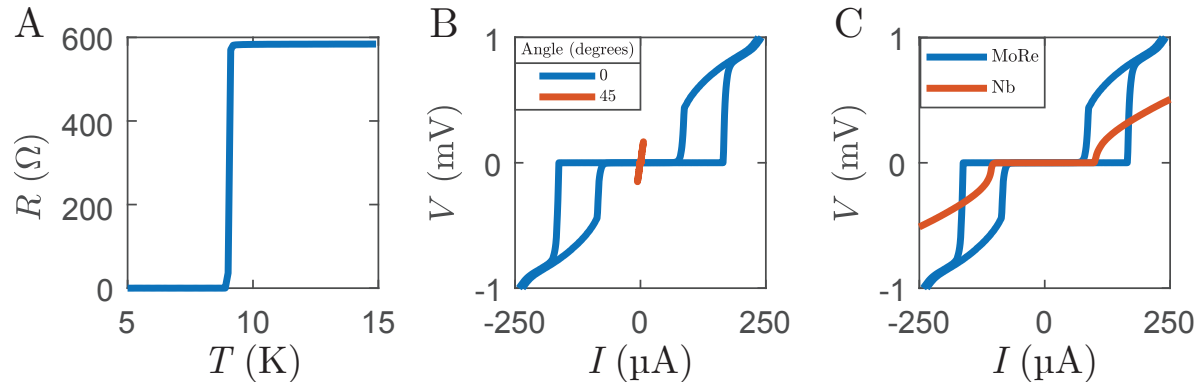

Figure 3.10: $T_{c}$ on MoRe thin film and $I-V$ measurements on MoRe-YBCO junctions. A) Resistance $R$ as function of temperature $T$. B) $I-V$ characteristics for junctions in different directions. C) Comparison between MoRe and Nb-based junctions.

\subsubsection{MoRe-based triangular $\pi$-loops}

Similar to the experiments in Section 3.5.2, we have performed magnetometry measurements on triangular $\pi$-loops. Figure 3.12 shows the SQUID voltage as a function of the applied current. We can immediately see that not only the lowest states $\left( \pm \frac{1}{2} \Phi_{0}\right)$ are stable, but also higher order states. At $I_{\text {bias }}=0$, we see that 4 states are stable, although one transition lies very close to zero. One would expect an even number of stable states, since the free energy $U$ is symmetric around $\Phi=0$. This means that there is a non-zero $\Phi_{e}$ that changes the energy landscape, and causes a shift of the magnetometry measurement along the horizontal axis.

Figure 3.11 shows an SSM image of a pair of triangular loops. We can clearly see that the lowerleft loop produces a stronger magnetic field than the upper-right one. This indicates that the lowerleft loop is in a higher state than the lower. By summing over both loops, we find a total flux of $2.5 \Phi_{0}$. Since both loops are in a positive
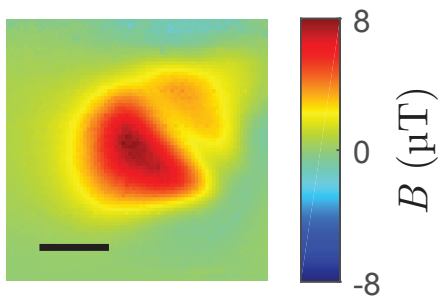

Figure 3.11: Pair of MoRe-based triangular $\pi$-loops, with the lower-left loop in a higher flux state than the upper-right. state, theoretically, the only possible combination should be that the lower-left loop is in the $+\frac{3}{2} \Phi_{0}$ and the upper-right in the $+\frac{1}{2} \Phi_{0}$ state, yielding a total flux of $2 \Phi_{0}$.

As before in the Nb-based triangular loops, part of this $\Phi_{e}$ is the sec- 

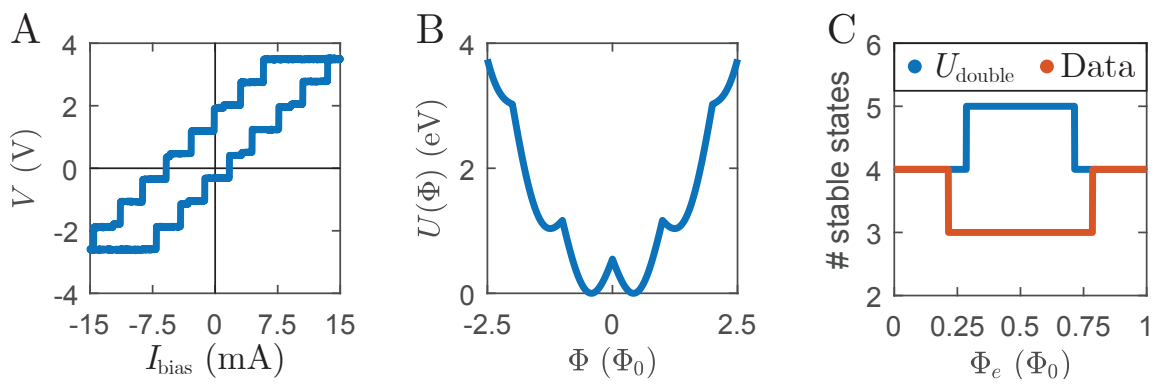

Figure 3.12: Magnetometry and energy analysis of triangular MoRe-based $\pi$ loops. A) SQUID voltage $V$ as function of applied current $I$. B) Free energy $U$ using the parameters extracted from the magnetometry. C) Comparison of the number of states stable as a function of external field as predicted by $U_{\text {double }}$ and the measurement data.

ond loop in the pair. However, the calculated influence of the other loop is approximately 0.02-0.03 $\Phi_{0}$, while the shift we observe in the magnetometry measurement is an order of magnitude larger $\left(\Phi_{e} \approx 0.3 \Phi_{0}\right)$. So it is unlikely that all of this can be attributed to the secondary loop. Since the design of the sample is the same as in Section 3.5, only with Nb substituted for MoRe, there are no other nearby structures that could have a significant influence. In addition, since the SSM setup is the same, we also expect no change in the influences from sources external to the setup. The exact origin of the increase in $\Phi_{e}$ is therefore uncertain.

We can again extract $\beta_{L}$ from the data. We find that $\beta_{L}=11$. Using $I_{c}=165 \mu \mathrm{A}$ from Figure 3.10 and the inductance $L=36 \mathrm{pH}$, we would expect $\beta_{L}=18$. The difference between our measured value and the expected value is again a factor 1.7 as in Section 3.5. This leads us to believe the simulated value for $L$ is off, since the error is practically the same.

With this we can calculate the free energy (shown in Figure 3.12B) and see that we should expect four stable states $\left( \pm \frac{1}{2} \Phi_{0}, \pm \frac{3}{2} \Phi_{0}\right)$ at zero bias, which matches the magnetometry (when corrected for the apparent external field). However, when looking at how the number of stable states changes with $\Phi_{e}$, we find that the number of stable states is either 4 or 5 , while based on the data in Figure 3.12A the number of stable states appears to be either 4 or 3 (see Figure 3.12C). This would imply a lower $\beta_{L}$ than what we extracted. 

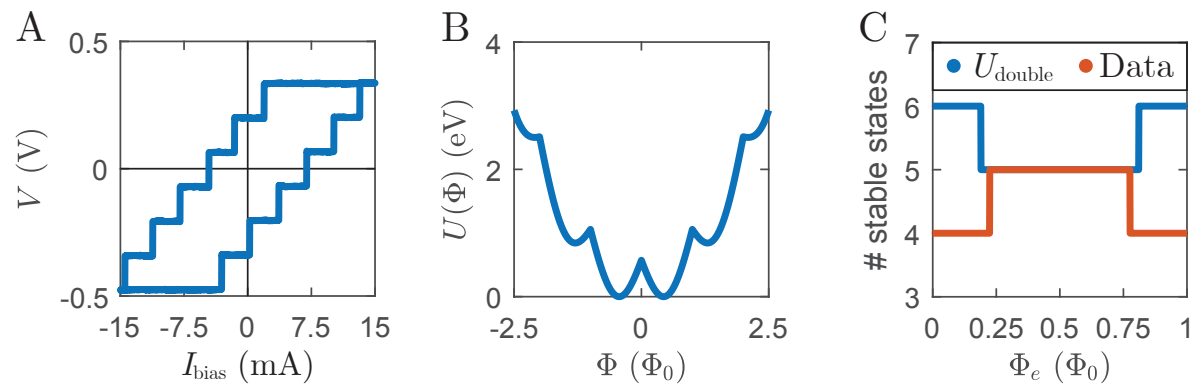

Figure 3.13: Magnetometry and energy analysis of square MoRe-based $\pi$-loops. A) SQUID voltage $V$ as function of applied current $I$. B) Free energy $U$ using the parameters extracted from the magnetometry. C) Comparison of the number of states stable as a function of external field as predicted by $U_{\text {double }}$ and the measurement data.

\subsubsection{MoRe-based square $\pi$-loops}

In addition to the triangular loops, we also performed measurements on square $\pi$-loops. The design of these squares is the same as in Section 3.5.1. The junctions on these squares are $6 \mu \mathrm{m}$ wide, while the junctions of the triangular loops as well as the junctions to determine $I_{c}$ are $3 \mu \mathrm{m}$ wide. Since we did not explicitly measure $I_{c}$ for a $6 \mu \mathrm{m}$ junction, we will for now simply assume $I_{c}$ doubles from the $3 \mu \mathrm{m}$ case, and is therefore $330 \mu \mathrm{A}$.

Figure 3.13 shows magnetometry results on these square loops. As in the triangular junctions, we also find both $\pm \frac{1}{2} \Phi_{0}$ and $\pm \frac{3}{2} \Phi_{0}$ states to be stable at $\Phi_{e}=0$. In addition, the $-\frac{5}{2} \Phi_{0}$ is also stable, though the transition from it to $-\frac{3}{2} \Phi_{0}$ lies very close to zero.

From the magnetometry, we can again extract $\beta_{L}$, which comes out to be 13.8. Using the simulated value of $L=25 \mathrm{pH}$ from Section 3.5.1 and $I_{c}=330 \mu \mathrm{A}$, we would expect $\beta_{L}=23$. This again is a factor 1.7 higher than our measured value.

We again see a significant shift along the horizontal axis, which stabilises the $-\frac{5}{2} \Phi_{0}$ state. We find the shift to be approximately $0.3 \Phi_{0}$, similar as with the triangular loops in the previous section. Since the square loop is not paired up like the triangular one and has no secondary loop nearby, this excludes the possibility that this $\Phi_{e}$ is due to a nearby loop.

With the extracted value for $\beta_{L}$ we can again calculate the free energy and look at the number of stable states, similar to what we did in Figure 
3.12. Figure 3.13B shows the free energy for $\beta_{L}=13.8$, while Figure $3.13 \mathrm{C}$ shows a comparison between the number of stable states based on the data

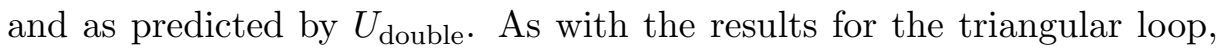
we find that these numbers do not match, due to $U_{\text {double }}$ again predicting a higher number of stable states.

\subsection{Discussion and conclusion}

In this chapter we have looked at $\pi$-loops based on hybrid high- $T_{c} /$ low$T_{c}$ Josephson junctions and their response to an applied magnetic field. Initially, the junctions were fabricated out of $\mathrm{Nb}$ and YBCO. Here we reproduced earlier findings that these loops spontaneously generate $\pm \frac{1}{2} \Phi_{0}$ of flux. Using the bias line to apply a local magnetic field, we were not only able to switch between $+\frac{1}{2} \Phi_{0}$ and $-\frac{1}{2} \Phi_{0}$ (Figure $3.7 \mathrm{~A}$ ), but also access higher flux states (Figure 3.7B). We also confirmed that these flux states follow $\left(n+\frac{1}{2}\right) \Phi_{0}$, by showing that each transition adds or removes $\Phi_{0}$ (Figures 3.7B and 3.9).

We then extracted $\beta_{L}$ from the magnetometry measurements and found a value between 6.1 and 6.4. This is significantly lower than the value we predicted, $\beta_{L}=11$, based on $I_{c}$ and a simulated value for $L$.

Since the triangular loops came in a pair, we attempted to measure the coupling between these loops. By manipulating the loops using the bias line, we performed magnetometry measurements on one loop while having the second loop in either the $+\frac{1}{2} \Phi_{0}$ or the $-\frac{1}{2} \Phi_{0}$ state (Figure $3.7 \mathrm{C}$ ). We observed a clear shift along the horizontal axis, showing that the second loop does indeed influence the behaviour of the first. We calculated the flux coupled from the second loop into the first to be $0.03 \Phi_{0}$, while a simulation of the situation gave a flux of $0.02 \Phi_{0}$.

To increase the number of stable states, we replaced the $\mathrm{Nb}$ part of the junctions with MoRe to increase the critical current and with that $\beta_{L}$. Our deposited MoRe has $T_{c}=9 \mathrm{~K}$, and the tested junctions have $I_{c}=165 \mu \mathrm{A}$. The MoRe-based junctions did show significant hysteresis, which we attributed to the increased $I_{c} R_{n}$ product and higher $\beta_{C}$.

In our magnetometry measurements we were again able to access higher flux states, but due to the higher $I_{c}$ of MoRe, we found more states to be stable. In both the triangular and square $\pi$-loops the $\pm \frac{3}{2} \Phi_{0}$ states were also stable, and in the square loops the $-\frac{5}{2} \Phi_{0}$ was stable as well. The asymmetry in the stability of states was found to be due to 
a significant $\Phi_{e}$ shifting the free energy, allowing the $-\frac{5}{2} \Phi_{0}$ state to be stable while the $+\frac{5}{2} \Phi_{0}$ was not.

An SSM image of this pair of triangles also confirmed the ability to stabilise higher flux states. Figure 3.11 showed that the loops were in clearly different, but both positive, states, we deduced to be the $+\frac{3}{2} \Phi_{0}$ state for one and the $+\frac{1}{2} \Phi_{0}$ state for the other. The total flux was calculated to be $2.5 \Phi_{0}$, which is higher than the expected $2 \Phi_{0}$. While we attempted to correct for the background field discussed prior, this is difficult since in theory, the flux through the loop should still be quantised. On the other hand, since we are not scanning at $h=0$, but at approximately $h=$ $5 \mu \mathrm{m}$, the screening of any background field is also not complete anymore. Another possible error is our value for the size of the pickup loop, which

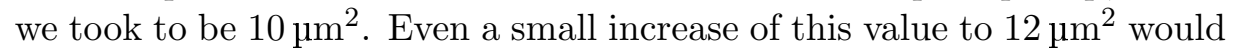
instead give a total flux of $2.1 \Phi_{0}$.

Again we extracted $\beta_{L}$ from the magnetometry measurements. For the triangular loop, we found $\beta_{L}=11$, again significantly lower than what we would have expected $\left(\beta_{L}=18\right)$ based on the critical current and simulation for $L$. Similarly for the square loop, we extracted a value of $\beta_{L}=13.8$, compared to the expected 23 .

Since the relative error in $\beta_{L}$ for both the Nb-based and MoRe-based triangular loops is 1.7, we attributed this error to the simulation of $L$. Although a change in $\beta_{L}$ can also be due to a change in $I_{c}$ in the loops compared to the single junctions, it would be too much of a coincidence if for both $\mathrm{Nb}$ and MoRe the change in $I_{c}$ from single junction to loop would be 1.7. An alternative is that our method for extracting $\beta_{L}$ (based on Figure 3.7E) is not correct, and it is through there we end up with a missing factor 1.7 .

For the MoRe square $\pi$-loop, the relative error in $\beta_{L}$ is larger, a factor 2. It is possible that again the simulated value for $L$ is off. Another possible error is in our assumption that, since the Josephson junctions in the square loops are twice as wide (6 $\mu \mathrm{m}$ compared to $3 \mu \mathrm{m})$, the critical current also doubles. While this assumption is true for small Josephson junctions, this does not hold in the wide junction regime $[57,58]$. Therefore, if our estimate for $I_{c}$ is too high, this would explain part of why the extracted value for $\beta_{L}$ is lower than expected.

Nonetheless, even with our extracted values of $\beta_{L}$, the free energy $U$ does not fully agree with our magnetometry measurement. The free energy predicts that the triangular $\mathrm{Nb}$-based $\pi$-loops are close to a transition from 
2 stable states to 4 . Yet our magnetometry measurement indicates that these higher states $\left( \pm \frac{3}{2} \Phi_{0}\right)$ still require approximately $0.7 \Phi_{0}$ of applied flux to stabilise. For the MoRe variant of the triangular loops, even though the magnetometry shows 4 stable states matching the prediction from the free energy, the latter predicts up to 5 stable states depending on $\Phi_{e}$, while the magnetometry shows either 3 or 4 stable states. Finally, magnetometry on the MoRe square loop indicates 4 or 5 stable states, while its free energy suggest 5 or 6 .

This means that there is a mismatch between our description of the free energy and the predicted number of stable states, and our method for extracting the value of $\beta_{L}$. The free energy diagrams consistently predict a higher amount of stable states than our magnetometry measurements indicate. As a check, we also calculated the free energy with the singlejunction model (see Reference [51]), but this model, too, overestimates the number of stable states, though its predictions are closer to the measurements than our double-junction model.

We can also work backwards from the magnetometry to find what the expression for the free energy should be. Using Figure 3.7E, if we imagine our system is at a transition point between 2 and 4 stable states, we can see that $2 I_{c} L=3 \Phi_{0}$. Similarly, at the transition from 4 to 6 stable states, $2 I_{c} L=5 \Phi_{0}$. Using this argument, the correct expression for the number of stable states should be 2 round $\left(\frac{\beta_{L}}{2 \pi}\right)$. This can be expressed as a corrected $\beta_{L}^{*}=\beta_{L}-\pi$. Using this, we can write down a corrected expression for the Josephson energy constant and the total free energy of the system:

$$
\begin{aligned}
E_{J}^{*} & =E_{J}-\frac{\Phi_{0}^{2}}{2 \pi L}, \\
U^{*}(\Phi) & =\frac{\left(\Phi-\Phi_{e}\right)^{2}}{2 L}-E_{J}^{*} \sqrt{2-2 \cos \left(\frac{2 \pi \Phi}{\Phi_{0}}\right) .}
\end{aligned}
$$

In Figure 3.14A we compare the corrected free energy $U^{*}$ with our original two-junction free energy $U_{\text {double }}$ (Equation 3.5). We can see that the general shape of the function is the same, with just slightly shallower minima. Figure $3.14 \mathrm{~B}$ shows the predicted number of stable states using $U^{*}$, compared to our deduced $2 \operatorname{round}\left(\beta_{L} / 2 \pi\right)$, and the original $U_{\text {double }}$. We see that $U^{*}$ matches $2 \operatorname{round}\left(\beta_{L} / 2 \pi\right)$ nicely, and by using the various values for $\beta_{L}$ from our experiments, find that this expression for the free energy more closely predicts the number of stable states that we observe (Figures 3.14C-E). 

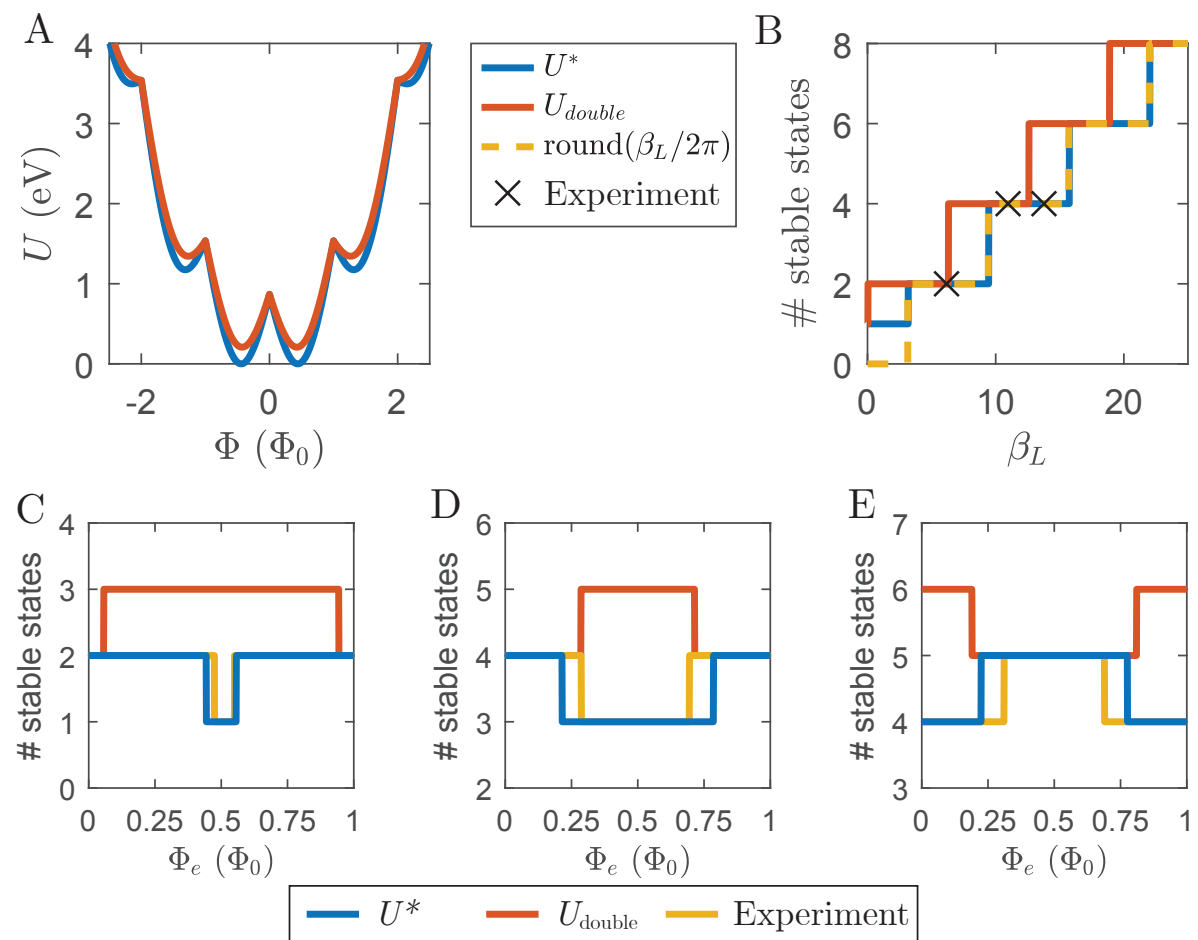

Figure 3.14: A) Comparison between the corrected expression for the free

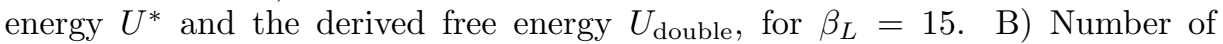
stable states predicted by $U^{*}, U_{\text {double }}$ and the deduced function $2 \operatorname{round}\left(\beta_{L} / 2 \pi\right)$. The black crosses indicate the experimentally observed values for $\beta_{L}$ and the associated value for the number of stable states. C-E) Comparison between the number of stable states based on $U^{*}, U_{\text {double }}$ and experimental data for the $\mathrm{C}$ ) Nb-based triangular loop; D) MoRe-based triangular loop; E) MoRe-based square loop. 
While performing the magnetometry measurements on the MoRe-based loops, we noticed a significant background field, approximately $0.3 \Phi_{0}$ (Figures 3.12 and 3.13). We excluded the possibility of this being due to a nearby loop, since the square loop has no other loops close enough to it to produce such values for $\Phi_{e}$. The second option of it being due to a field originating from outside the setup can also be excluded, since the sample and sensor are in a shielded space (see Chapter 2, Section 2.2).

An option is that the sensor itself plays some role in this. While there can be a fluxoid trapped in the SQUID, since its inductance is low, it will produce negligible magnetic field. The second option is that there is a vortex trapped in the superconducting material that the SQUID is made out of (such as in the pickup loop). If we calculate the flux through a $16 \mu \mathrm{m} \times 16 \mu \mathrm{m}$ square (roughly the size of our structures) using the field of a vortex placed $5 \mu \mathrm{m}$ above it (see Equation 2.17), we find $\Phi_{e} \approx 0.5 \Phi_{0}$. This is in the right order of magnitude to explain our observations. This would also explain why we did not observe it during the measurements on the Nb-based loops, since the vortex might not be present during every cooldown of the system. 


\section{4}

\section{Scanning SQUID microscopy on dipole-based systems}

In a perfectly symmetric set of Maxwell's equations, the magnetic monopole would be the equivalent of the electron. However, at this time, magnetic monopoles exist only as a theoretical exercise or as emergent effects (such as in spin ice materials); no particles with a magnetic charge have been conclusively proven to exist. Because of this, the most elemental magnetic object is the magnetic dipole. This chapter focuses on the imaging and analysis of objects that exhibit primarily dipolar behaviour.

I would like to thank the Ariando group of the National University of Singapore for their Pt-capped $\mathrm{BaTiO}_{3}$ films on $\mathrm{SrTiO}_{3}$, as well as Daan Wielens, M.Sc. for use of his structured V-BST films, both described in Section 4.2. I also want to thank Dr. Pegah Mirzadeh of the University of Aveiro for our collaboration on the $\mathrm{Fe}_{3} \mathrm{Se}_{4}$ nanoparticles described in Section 4.3. Finally I would like to thank Dr. Lennart de Groot and Annemarieke Béguin, M.Sc. of Utrecht University, Dr. Karl Fabian of the Geological Survey of Norway and Dr. Auke Barnhoorn of the Delft University of Technology for their collaboration involving the geomagnetic samples in Section 4.4, which was published as Lennart V. de Groot et al., Determining individual particle magnetizations in assemblages of micrograins, Geophysical Research Letters vol. 45, 2017. 


\subsection{Dipole theory}

The magnetic point dipole is the first non-zero term in the magnetic multipole expansion. The magnetic vector potential $\vec{A}$ at a point $\vec{r}$ due to a current $I$ along a curve $C$ at point $\vec{r}^{\prime}$ is given by:

$$
\vec{A}(\vec{r})=\frac{\mu_{0} I}{4 \pi} \int_{C} \frac{1}{\vec{r}-\vec{r}^{\prime}} d \vec{l},
$$

where $\mu_{0}$ is the magnetic permeability of the vacuum. The term $1 /\left(\vec{r}-\vec{r}^{\prime}\right)$ can be expanded in a Taylor series, known as the multipole expansion:

$$
\frac{1}{\vec{r}-\vec{r}^{\prime}}=\frac{1}{\vec{r}} \sum_{n=0}^{\infty}\left(\frac{\vec{r}^{\prime}}{\vec{r}}\right)^{n} P_{n}(\cos \theta),
$$

where $P_{n}$ is the $n^{\text {th }}$ Legendre polynomial and $\theta$ the angle between $\vec{r}$ and $\vec{r}$. The $n=0$ term (which would be the monopole term) results in 0 , since the use of a vector potential requires $\nabla \cdot \vec{B}=0$ and therefore already assumes the absence of magnetic monopoles. For $n=1$, we obtain

$$
\begin{aligned}
& \vec{A}(\vec{r})=\frac{\mu_{0}}{4 \pi} \frac{(\vec{m} \times \vec{r})}{r^{3}}, \\
& \vec{m}=\frac{\mu_{0} I}{8 \pi} \int_{C} \vec{r} \times d \vec{\ell},
\end{aligned}
$$

where $m$ is the magnetic moment. Using $\vec{B}=\nabla \times \vec{A}$, we obtain the magnetic field:

$$
\vec{B}=\frac{\mu_{0}}{4 \pi}\left[\frac{(3 \vec{r}(\vec{m} \cdot \vec{r}))}{r^{5}}-\frac{\vec{m}}{r^{3}}\right] .
$$

Since typically $r \gg r^{\prime}$, higher order terms of the vector potential can be ignored since $\left(r^{\prime} / r\right)^{n} \approx 0$ for larger $n$. In the context of scanning SQUID microscopy, this would mean that magnetic particles significantly smaller than the spatial resolution can be described as a point dipole. Because of this, data can be fit to equation 4.5 to extract $\vec{m}$, which can give valuable information about the material under investigation.

The point dipole field was used earlier in Chapter 2 to define the spatial resolution of a scanning SQUID system. Here we will have a more detailed look at how magnetic dipoles can manifest themselves in SSM images. 
A

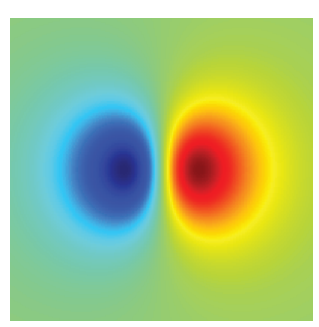

B

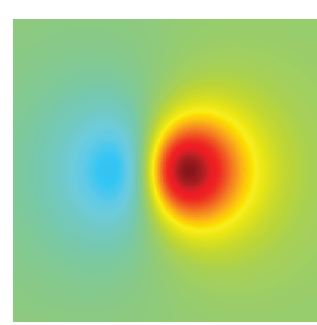

C

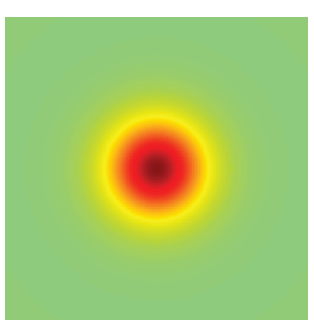

Figure 4.1: Simulated SSM images of point-dipoles in different orientation: A) fully along the $x$-axis; B) at a $25^{\circ}$ angle with the $x y$-plane; C) fully along the $z$-axis.

The classic dipole image is the double-lobe image as presented in Figure $4.1 \mathrm{~A}$. One half represents the magnetic field pointing away from the source, the other the field pointing towards it. For such a dipole, the magnetic moment lies in the $x y$-plane. While the SSM is ideally only sensitive to the out-of-plane component of the magnetic field (the component perpendicular to the pickup coil), in-plane dipoles can readily be imaged due to the closed-loop nature of magnetic field lines (i.e., the magnetic field lines have to bend around to close, giving them a non-zero $z$-component).

Of course, an ideal isotropic magnetized particle can have its magnetic moment oriented along any direction in three dimensions. Figure 4.1B shows the field of a point dipole at a $25^{\circ}$ angle as it would be imaged by SSM. We can see in Figure 4.1C that in extreme cases, with the moment aligned fully along the $z$-axis, only a single lobe is visible, giving it an almost monopole-like appearance.

\subsection{Magnetic pollution}

When dipoles are encountered in SSM, they can often be attributed to magnetised dirt particles that have adhered to the sample surface. Most samples go through various stages of fabrication, handling and transport, leading to exposure to ambient conditions. Fine Fe-based residue from equipment, experimental setups or tweezers can also be a source of magnetic pollution. Since these particles will often have a non-spherical shape, shape anisotropy will cause the magnetic field to align itself along the long axis of the particle. This axis will also often lie in the $x y$-plane (i.e., the particle lies 'flat' on the surface), which means most magnetic pollution 


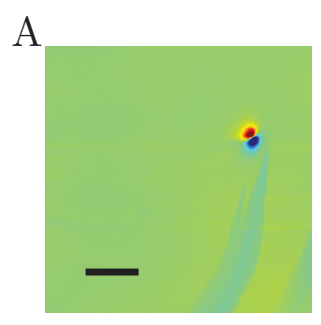

$\mathrm{D}$

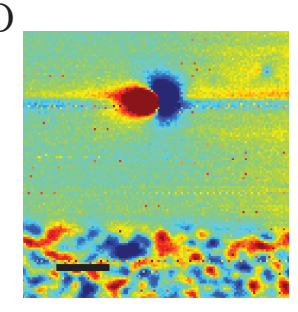

$\mathrm{B}$

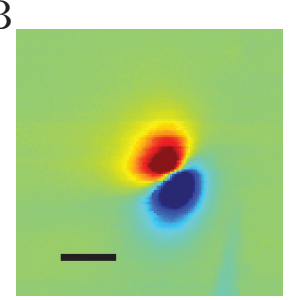

E

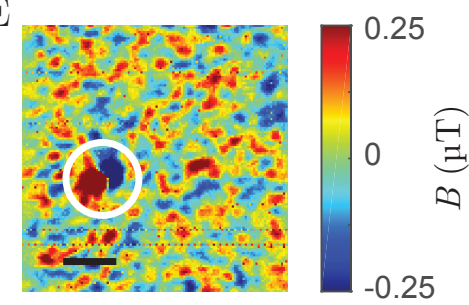

幽

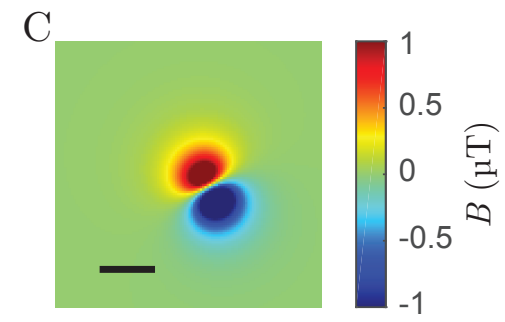

Figure 4.2: Contaminant on $\mathrm{BaTiO}_{3}$ with Pt capping layer. A) SSM scan
containing a single dipole-like feature. Scale bar indicates $200 \mu \mathrm{m}$. B) Zoom of the feature in (A). C) Result of fitting the data in (B) to Equation 4.5. D) Scan near a structured edge on a V-BST film. E) Scan on a V-BST film. A possible contaminant is highlighted in white. Scale bars in (B-E) indicate $50 \mu \mathrm{m}$.

will have its magnetic moment largely in the $x y$-plane.

Figure 4.2 shows $\mathrm{SSM}$ data on a $\mathrm{BaTiO}_{3}$ thin film on $\mathrm{SrTiO}_{3}$, with a thin Pt capping layer. Figure $4.2 \mathrm{~A}$ shows a $1000 \mu \mathrm{m} \times 1000 \mu \mathrm{m}$ scan. We can see that most of the surface is not magnetically active, apart from a single dipole-like feature, highlighted in Figure 4.2B. The typical doublelobe structure is clearly visible, meaning the dipole moment is oriented (mostly) in-plane.

We can fit the data of Figure 4.2B to the dipole equation (Equation 4.5). By doing this, we can extract the magnetic moment $m$ of the particle. The fitting result is shown in Figure $4.2 \mathrm{C}$, and matches the measurement data in Figure 4.2B well. The extracted moment is $\vec{m}=\left\{m_{x}, m_{y}, m_{z}\right\}=$ $\{-1.49,2.86,-0.56\} \times 10^{10} \mu_{\mathrm{B}}$, with a total moment of $3.27 \times 10^{10} \mu_{\mathrm{B}}$. This would, for example, correspond to an Fe particle with diameter $\approx$ $2 \mu \mathrm{m}$. We find most contaminants (across different samples from different labs) to lie in the range $m=10^{8}-10^{10} \mu_{\mathrm{B}}$. Considering the spatial and magnetic field resolution of our setup, the lower limit is a particle of $m \sim 10^{7} \mu_{\mathrm{B}}$.

Contaminants are most easily visible on samples with little-to-no mag- 
netic activity such as Figure 4.2. In samples that produce considerable magnetic fields, it becomes harder to detect contaminants as their magnetic field becomes small compared to that of the sample. Structured samples could give some insights: if parts of the sample that have no magnetic material show contaminants, chances are that the magnetically active areas do as well. As an example, Figure 4.2D shows a scan on a structured V-doped $\mathrm{BiSbTe}_{3}$ (V-BST) sample. The lower part of the figure shows the magnetically active V-BST, while the upper part shows an area where the material has been removed. A contaminant is visible in this upper area. Because of this, we can look out for contaminants in the V-BST areas (Figure 4.2E). Areas containing such contaminants can then be removed from the analysis procedure, allowing for improved analysis of the material of interest itself.

\subsection{Measurements on $\mathrm{Fe}_{3} \mathrm{Se}_{4}$ nanoparticles}

Magnetic nanoparticles are not just interesting to study fundamental magnetism in low-dimensional systems but also for potential applications like magnetic markers in a medical context (for example, see References [59, 60]). In this section, we present measurements on ferrimagnetic $\mathrm{Fe}_{3} \mathrm{Se}_{4}$ nanoparticles $\left(T_{C}=335 \mathrm{~K}[61]\right)$. As mentioned in Section 4.1, the magnetic field of magnetic objects much smaller than the resolution of the SSM can be described as a point dipole. These $\mathrm{Fe}_{3} \mathrm{Se}_{4}$ nanoparticles therefore lend themselves excellently to do just that. We will also use these particles to experimentally verify the spatial resolution of the SSM setup.

\subsubsection{Sample fabrication}

The $\mathrm{Fe}_{3} \mathrm{Se}_{4}$ nanoparticles were synthesised using thermal decomposition of Fe and Se precursors (for more details, see References [62-64]). The nanoparticles were then dispersed onto a $\mathrm{Si}$ substrate with a $6 \mu \mathrm{m} \mathrm{SiO}_{\mathrm{x}}$ top layer. Acetone was used as a dispersive medium. The sample was then magnetised, for practical reasons, using $\mathrm{Nd}$ permanent magnets with a surface field strength of $0.5 \mathrm{~T}$, placed $5 \mathrm{~mm}$ apart along the $x$-direction, at room temperature. 

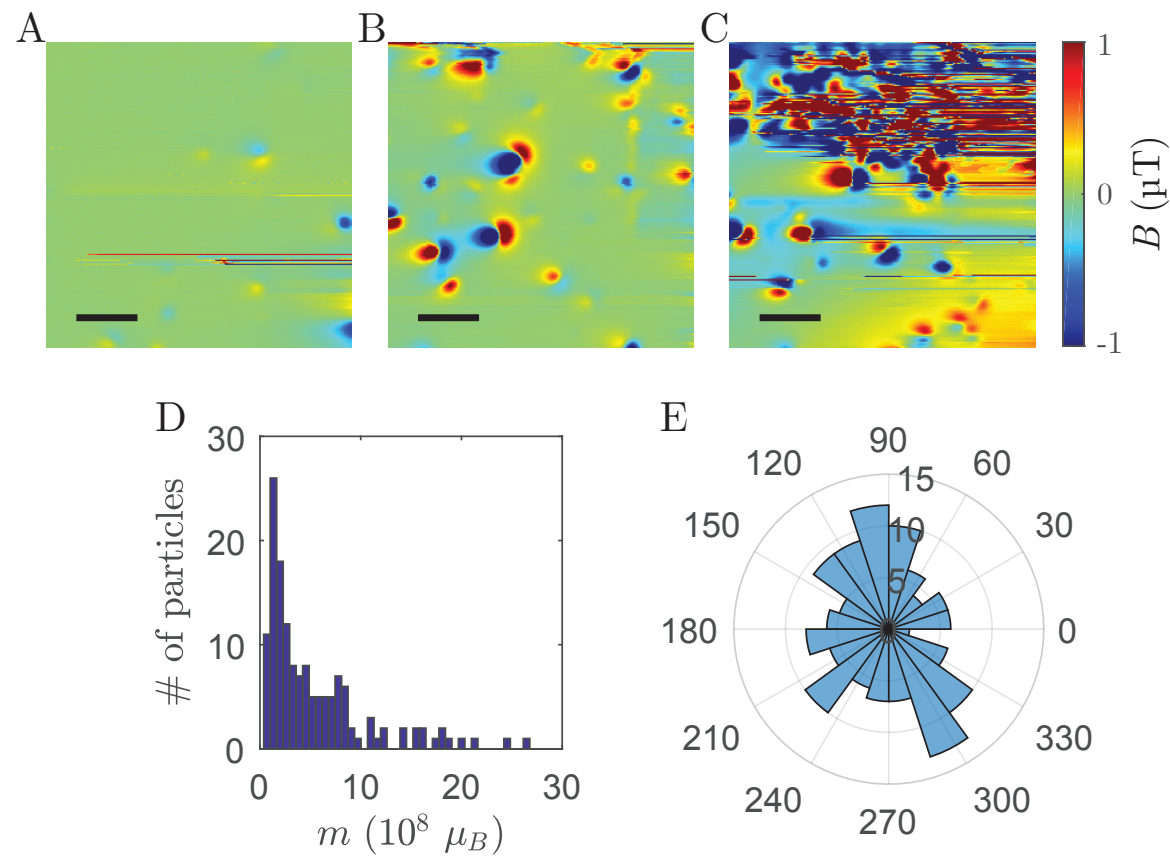

Figure 4.3: $\mathrm{SSM}$ measurements and analysis of $\mathrm{Fe}_{3} \mathrm{Se}_{4}$ nanoparticles. A-B) Representative $250 \mu \mathrm{m} \times 250 \mu \mathrm{m}$ SSM scans. C) Distribution of extracted magnetic moments. D) Distribution of extracted direction of the magnetic moments.

\subsubsection{Results and discussion}

Figures 4.3A-C show three $250 \mu \mathrm{m} \times 250 \mu \mathrm{m}$ scans, representative of the $1 \mathrm{~mm} \times 1 \mathrm{~mm}$ area that was imaged. As we can see, there are many objects visible that produce dipole-like magnetic fields. While some are relatively isolated, we can also see a high amount of clustering of particles in certain areas (like the upper area of Figure 4.3C).

To extract information about the particles, we again fit the SSM data to the dipole equation (Equation 4.5) as we did in Section 4.2. Figures 4.3C and D show data on 148 particles. Figure 4.3C shows the distribution of magnetic moments. The moments lie in a range between $3.7 \times 10^{7}$ and $3.3 \times 10^{9} \mu_{\mathrm{B}}$, while the distribution peaks around $1.5 \times 10^{8} \mu_{\mathrm{B}}$. Assuming a disk-shaped particle with a $300 \mathrm{~nm}$ diameter and $100 \mathrm{~nm}$ thickness [64], $1.5 \times 10^{8} \mu_{\mathrm{B}}$ would correspond to roughly $5.8 \mu_{\mathrm{B}}$ per unit cell. This is significantly higher than $2.2 \mu_{\mathrm{B}}$ per unit cell found in earlier experiments 
[65] or the $4.2-4.3 \mu_{\mathrm{B}}$ per unit cell based on theoretical models $[65,66]$. This could be related to the SSM data: for example, an incorrect sensor calibration. Another option is that our estimated size of the nanoparticles is incorrect (i.e., too small).

Figure 4.3D shows the distribution of the direction of the magnetic moments in the $x y$-plane. Although the applied magnetic field to magnetise the sample was along the $x$-direction, the actual direction of the magnetic moments appears to be distributed randomly in the $x y$-plane. This might be because the field used to magnetise the sample was below the coercive field at room temperature, or because room temperature gives the particles enough thermal energy to reorient their magnetic moment. We do find most moments to have their largest component in the $x y$-plane, which can be attributed to the disc-like shape of the particles (where an out-of-plane magnetic moment would mean the particle is standing on its edge).

The biggest obstacle during this analysis is the large amount of particles that were clustered together. Because of this, we cannot fit them to the dipole model and extract their magnetic moments. It is therefore hard to say how representative the data in Figures $4.3 \mathrm{C}$ and D is for all particles. One might imagine, for example, that larger particles might cluster due to their stronger magnetic interaction, leaving smaller particles isolated on the sample.

\subsubsection{Determining the spatial resolution}

As described in Chapter 2, the resolution of an SSM system can be defined as the distance between the two extrema of an imaged point dipole. Since our spatial resolution is on the order of $1-10 \mu \mathrm{m}$ and the nanoparticles have dimensions on the order of $100 \mathrm{~nm}$, they can be described fairly accurately as point dipoles. Therefore, we can use the data on these nanoparticles to determine the spatial resolution. Figure 4.4 shows the distribution of

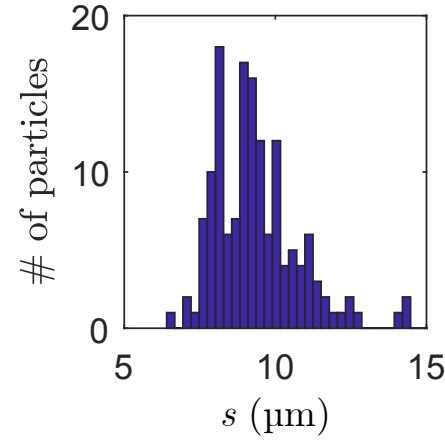

Figure 4.4: Distribution of the distance $s$ between the two extrema of the analysed particles. 
distance $s$ between the two extrema of the analysed particles. As we can see, the distribution peaks around $9 \mu \mathrm{m}$, meaning this would be our spatial resolution.

We can see some particles do have a smaller value for $s$ than the value we set for our spatial resolution. One could argue that the spatial resolution should be equal to the smallest value of $s$. However, these data points are likely a combination of noise and the step size $(=1 \mu \mathrm{m})$, meaning that for some measured dipoles, the highest and/or lowest values in the data do not correspond to where those points ought to be based on the height and pickup loop size of the sensor, leading to errors in $s$. We therefore take the peak of the distribution to be the spatial resolution, rather than the lowest value.

\subsection{Palaeointensity measurements}

Determining the palaeofield, the direction and magnitude of the Earth magnetic field throughout Earth's history, is vital for creating a proper model of the inner workings of Earth's magnetic field. These experiments involve measuring geological samples containing iron oxides, whose magnetisation is dominated by Earth's field at the time of their creation. However, these measurements are destructive and unreliable, making data analysis and modelling difficult. Here, we investigate whether SSM can play a role in developing a novel method to measure and analyse these samples.

\subsubsection{Introduction}

Palaeomagnetism is the study of Earth's magnetic field and how it has changed throughout Earth's history. This is mostly done through analysing rock, sediment or even archaeological artifacts. Dating methods such as carbon-14 dating are used to determine the formation date of the sample while magnetometry measurements yield information about the magnetic moment of the sample. Careful analysis of the magnetic moment can in turn detail the direction and strength of the Earth's magnetic field when the sample formed. Finally, by obtaining samples from different places around the globe, a snapshot of the field can be made, which can be used to model the inner workings of the field's source and predict future changes. 
The magnetic information is mostly carried by iron oxides. As samples form (e.g., as lava cools), iron oxide particles are magnetised by the Earth's magnetic field as they cool through their Curie temperature $T_{C}$. Assuming then that the sample is not later reheated above $T_{C}$ or exposed to some strong magnetic field, it will hold this magnetic moment (as described by Néel's theory [67,68]). This is known as the thermoremanent magnetisation. It is then mostly influenced by shape anisotropy and multi-domain behaviour, since these particles will vary greatly in size and shape. For this reason, reliable data can only be gathered using large collections $\left(>10^{6}[69]\right)$ of magnetised particles in samples.

The current leading method for determining these magnetic moments is the Thellier-Thellier method [70]. It involves a repeated cycle of heating the sample to a higher temperature each cycle, thereby removing part of the magnetisation, then cooling in an applied field to remagnetise it. The basic idea is that the ratio between the magnetisation of the sample before and after the cycle equals the ratio between the original (i.e., Earth's) magnetic field and the applied field.

Although it is the current leading method, the Thellier-Thellier approach has two disadvantages. The first is that the method is destructive in nature. Heating the sample above $T_{C}$ means the original magnetic moment is lost. As a result, the measurement cannot be repeated, for instance in case verification is needed. The second disadvantage is that the results from the measurement are not always reliable, since many particles exhibit multi-domain behaviour and therefore violate the assumptions underlying Néel's theory [71]. Because of this, a measurement method that solves at least one of these two problems is highly sought after.

in this section we will investigate such an alternative. Instead of measuring a bulk sample, we use SSM to measure the magnetic field produced by individual particles. This would allow for selection of those particles that abide by Néel's theory and are therefore reliable recorders of the palaeofield. To reduce the number of degrees of freedom in the inversion process, we include X-ray computed tomography (XCT) measurements to obtain positional data for the magnetic particles. This reduces the number of degrees of freedom in the dipole equation from 6 ( 3 for $\vec{r}, 3$ for $\vec{m}$ ) down to just 3 . In addition, having the positional degrees of freedom fixed guarantees the uniqueness of the inversion [72]. We test this method on an artifical sample as a proof-of-concept. 


\subsubsection{Sample fabrication}

As mentioned, we fabricated an artificial sample in order to control the density of particles to show the functionality of the method. The magnetite grains used are of natural origin and range in size between 5 and $35 \mathrm{\mu m}$ (see Reference [73] for typical properties of such particles). The magnetite is mixed with calcite powder in a 1:10 magnetite:calcite ratio to prevent the magnetite grains clumping together due to mutual magnetic attraction. This in turn is mixed with Araldite epoxy and cast into a Stycast epoxy holder in a disk of roughly $1 \mathrm{~mm}$ diameter and $65 \mathrm{\mu m}$ thickness. The sample ended up containing 132 magnetite grains, yielding a particle density of $2800 \mathrm{~cm}^{-3}$, approximately 1 order of magnitude smaller than in natural rock samples. For optimal SSM scanning, the surface is polished using a silicon colloid suspension designed to minimise surface strain.

For the initial SSM data, the natural magnetic moment of the magnetite grains was used. That is to say, no external field was applied to the sample to magnetise it, though the moments may have been influenced by the various fabrication and preparation steps. This state will be referred to as the 'unmagnetised' state. For a second set of measurements, we magnetised the sample in an asymmetric alternating field (amplitude of $300 \mathrm{mT}$, dc bias of $40 \mu \mathrm{T}$ ) at a $45^{\circ}$ angle with the surface normal. The dc bias roughly corresponds to the strength of the Earth's magnetic field. We will refer to this state as the 'magnetised' state.

\subsubsection{X-ray computed tomography results}

First, the positional data for the grains was obtained with XCT, performed at the Technical University of Delft. 2000 X-ray images were made in $0.18^{\circ}$ steps, covering the full $360^{\circ}$. By choosing the proper density cutoff, we can discern between the magnetite grains $\left(\sim 5150 \mathrm{~kg} \cdot \mathrm{m}^{-3}\right)$, the calcite $\left(\sim 2710 \mathrm{~kg} \cdot \mathrm{m}^{-3}\right)$ and the two epoxies (Araldite: $\sim 1170 \mathrm{~kg} \cdot \mathrm{m}^{-3}$; Stycast: $\left.\sim 2290 \mathrm{~kg} \cdot \mathrm{m}^{-3}\right)$. Figure $4.5 \mathrm{~A}$ shows the location of the 132 grains in the sample projected onto the $x y$-plane. Most grains fall within the $\sim 1 \mathrm{~mm}$ diameter as designed.

The resolution of the XCT is about $0.35 \mathrm{\mu m}^{3}$. This means that the largest grains consist of up to 80,000 voxels. As is, the inversion step would require too much computing time. To reduce the number of computations, voxels are grouped into cuboids (Figure 4.5B). This is done in such a way that, at each step, the remaining volume of a grain is filled with the largest 

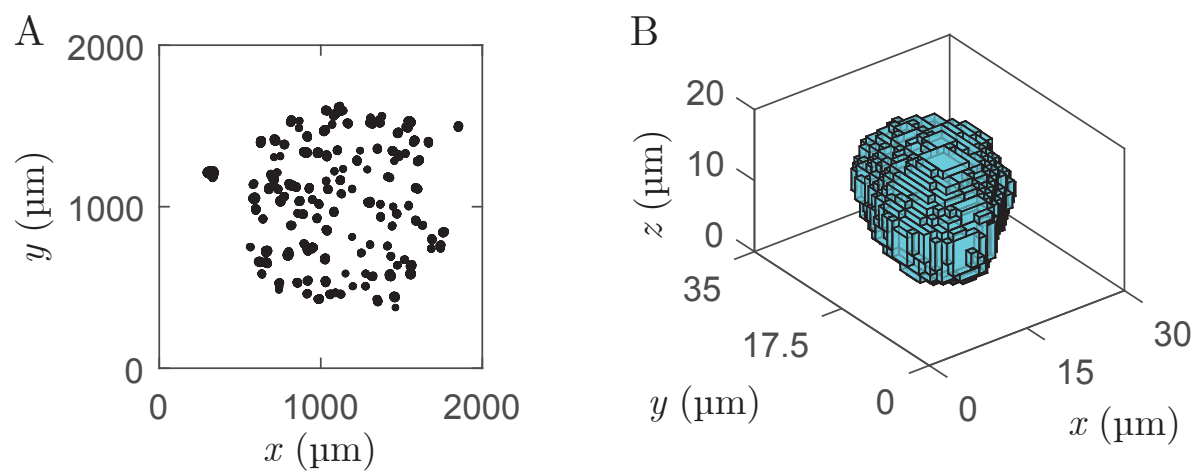

Figure 4.5: X-ray computed tomography results of the sample. A) Location of the magnetite grains in the sample, projected onto the $x y$-plane. B) A single magnetite grain represented as a collection of cuboids.

possible cuboid. During the inversion procedure, each cuboid is treated as a single dipole. By doing this, inversion calculations are sped up by a factor of 10-100.

\subsubsection{Results from the unmagnetised state}

The first set of scans was performed on the sample without any explicit magnetisation. Figure $4.6 \mathrm{~A}$ shows a scan of the full area containing the magnetite grains. As with the XCT data in Figure 4.5A, we see that the magnetic activity is similarly confined to a $\sim 1 \mathrm{~mm}$ disk. We can also see some scarring in the data, due to the slew rate of the SSM feedback system not being large enough to keep up with the large changes in magnetic field.

As mentioned before, the large amount of XCT and SSM data makes inversion computationally demanding. Therefore, we opted to analyse sections of the data instead of the full scan. One such section is highlighted in Figure 4.6A and shown in Figure 4.6B. The dipole nature of the individual grains can be seen, though it is sometimes obscured in clusters of particles (for example, at particles 2, 3 and 4). The particles also appear to have a large range of magnetic moments, as can seen by comparing the fields of particles 1 and 6 to the rest.

By carefully placing the XCT data from Figure 4.5 over the SSM data (indicated in white in 4.6B), we can fit the SSM data to the dipole equation using the positional data from the XCT. The result of this fit is shown in Figure 4.6C. We can see that the fitting result matches the SSM data 

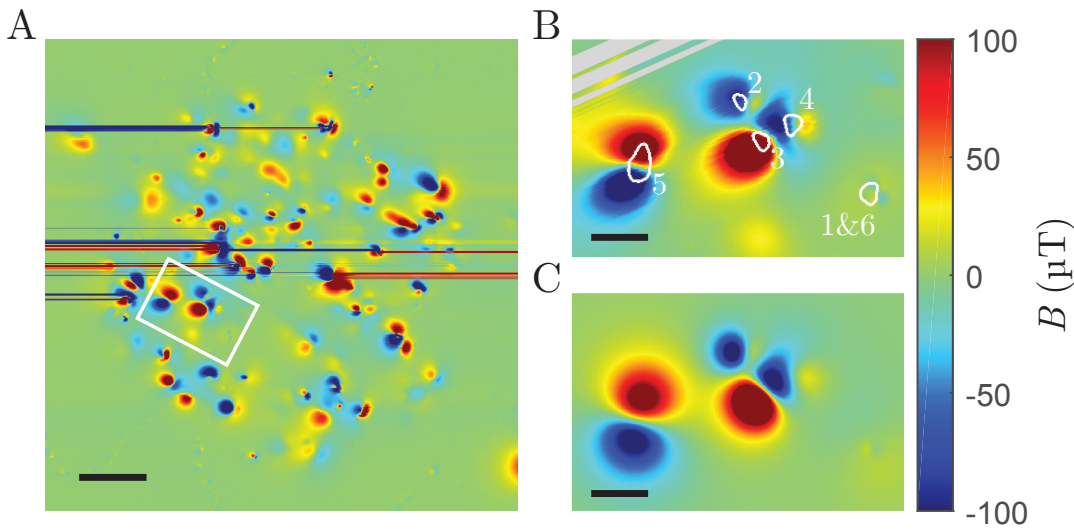

Figure 4.6: SSM data on the sample in the unmagnetised state. A) Large area scan. Scale bar indicates $200 \mu \mathrm{m}$. B) Section of the full scan highlighted in white used for analysis. Scale bar indicates $50 \mu \mathrm{m}$. The locations of the individual grains from the XCT data are marked in white. C) Fit result using the data of (B) and the XCT data. Scale bar indicates $50 \mu \mathrm{m}$. The locations of the individual grains from the XCT data are marked in white.

quite well. Because of the assumption that the SSM is only sensitive to the $z$-component of the magnetic field, the slight leftward smearing (owing to the sensor being at an angle) seen in the scanning data is not reproduced in the fit.

From the fit we can extract the magnetisations of the individual particles, shown in Figure 4.7. We analysed 20 particles in total, yielding a mean magnetisation of $7.1 \mathrm{kA} \cdot \mathrm{m}^{-1}$ and a range of 0.4 to $92.2 \mathrm{kA} \cdot \mathrm{m}^{-1}$. We also see that the magnetisation decreases with increasing particle size. This could indicate larger particles forming multiple magnetic domains which can partially cancel each other's magnetic field. We find that the orientation of the individual grains appears to be random, which is what we would expect since we did not apply an external magnetic field to magnetise the sample.

Additionally, we find that for particles close to the sample surface $(<5 \mu \mathrm{m})$, the difference between the data and the fit is usually larger than for lower-lying particles. It is possible that the magnetic field from these particles as measured by SSM is dominated by multi-domain behaviour, which is more apparent for nearby particles and becomes averaged out for deeper particles. Therefore, these particles cannot be modelled as dipole 

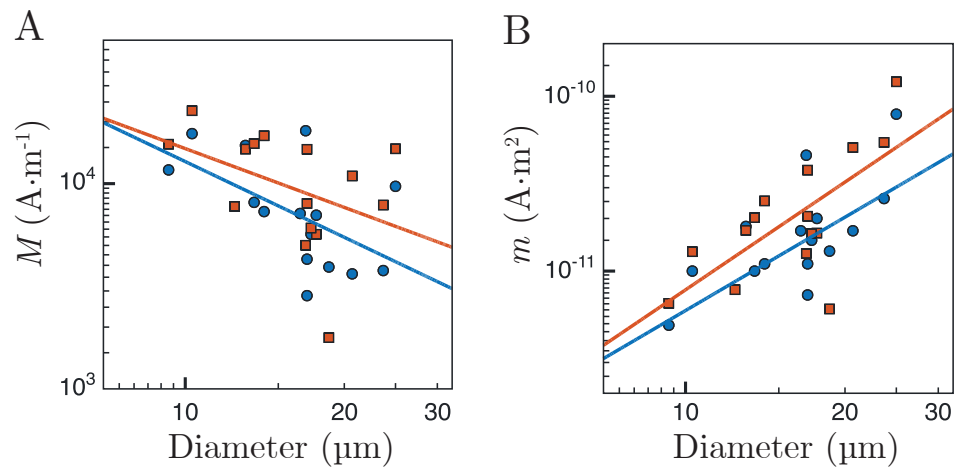

C

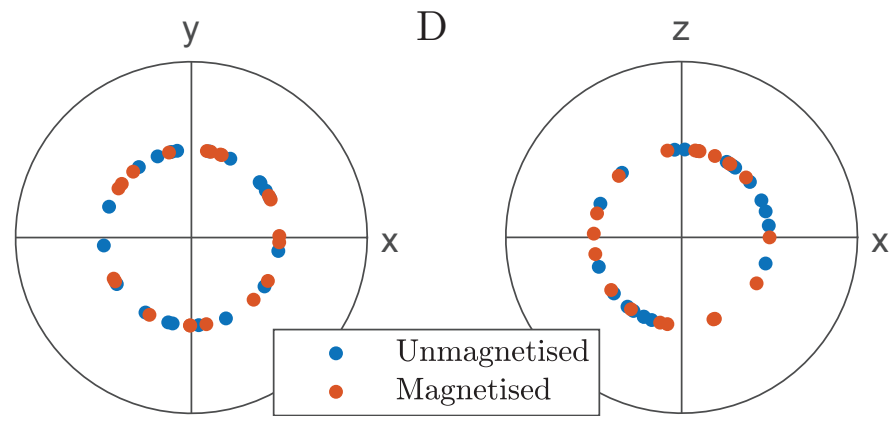

Figure 4.7: Analysis of magnetic moments of several particles. A) Magnetisation and B) magnetic moment versus particle diameter of the analysed particles. The lines indicate linear fits through the data. C) In-plane and D) out-of-plane angle of the magnetic moment of the analysed particles.

structures and we rejected these particles from our analysis.

\subsubsection{Results from the magnetised state}

After the first set of measurements, we magnetised the sample using an external field as described in Section 4.4.2. We then repeated the SSM measurement to analyse the changes in the magnetisation of the particles. These scan results are shown in Figure 4.8.

Figure 4.8A again shows a large-area scan of the sample, with the same section highlighted in $4.8 \mathrm{~B}$. We can clearly see that the magnetic moment of the particles has changed due to the externally applied field. As before, combining the SSM data with the XCT data allows us to calculate the magnetic moments for each particle, yielding the image in $4.8 \mathrm{C}$. The fit again matches the SSM data well, though again does not properly capture 

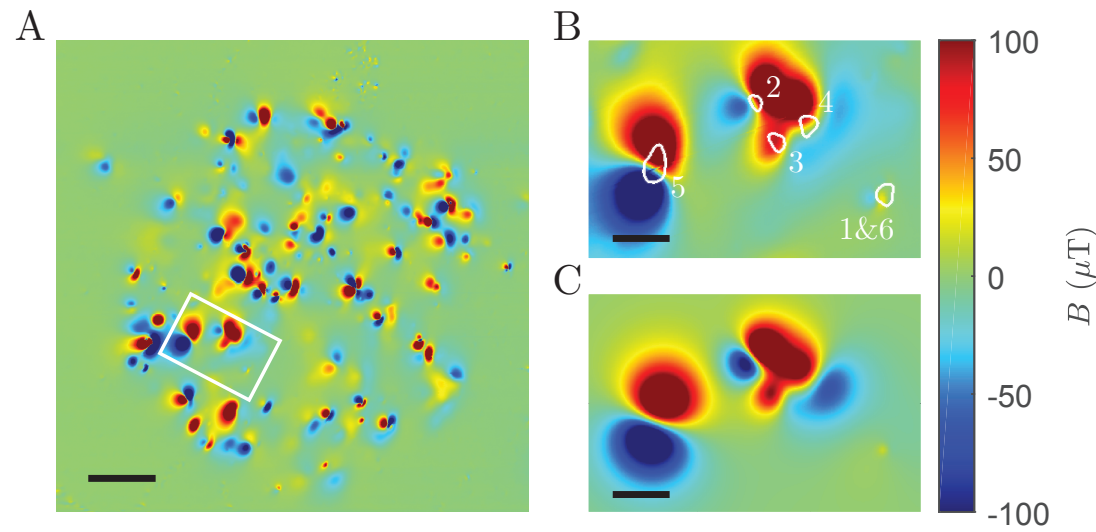

Figure 4.8: SSM data on the sample in the magnetised state. A) Large area scan. Scale bar indicates $200 \mu \mathrm{m}$. B) Section of the full scan highlighted in white used for analysis. Scale bar indicates $50 \mu \mathrm{m}$. The locations of the individual grains from the XCT data are marked in white. C) Fit result using the data of (B) and the XCT data. Scale bar indicates $50 \mu \mathrm{m}$. The locations of the individual grains from the XCT data are marked in white.

the influence of the sensor angle.

By analysing the same particles as in Section 4.4.4, we can compare the new magnetisations to the old. We find that on average the magnetisation has increased by about $77 \%$, ranging from 0.4 to $132 \mathrm{kA} \cdot \mathrm{m}^{-1}$ with a median of $9.5 \mathrm{kA} \cdot \mathrm{m}^{-1}$ (see also Figure 4.7 ). As before, the magnetisation decreases with increasing particle size, which may be indicative of multi-domain behaviour. However, in spite of applying an external field with a well-defined direction, the direction of the magnetisation for the particles still appears to be random. This may indicate that the direction of the magnetic moment in these particles is largely influenced by shape anisotropy.

\subsubsection{Discussion and conclusion}

In summary, we have shown that SSM can play an important role in a new method that non-destructively evaluates the magnetic moment of individual particles in an assemblage. By combining SSM with XCT data, the inversion becomes not only unique but also computationally feasible.

This study was performed on an artificial sample with a lower particle density than expected in actual lava deposits. An XCT scan on a volcanic 
sample showed a roughly 20 times higher particle density. Nonetheless, due to the high resolution of both SSM and XCT, the dataset should still be significantly overdetermined ( $>100$ data points per variable). If we were then to scale up the sample such that it would contain the required $10^{6}$ particles, the total area to be imaged would be $<3 \mathrm{~cm}^{2}$. Such areas, while time-consuming, are well within the upper limits of SSM or other scanning magnetometers, meaning the bottleneck of this method would still be the computational load of the inversion.

Still, the significantly increased amount of particles will make it harder to match the XCT data with the SSM scans. For this artificial sample, finding the correct placement of the XCT data with respect to the SSM data was relatively straightforward due to the limited area and number of particles. One way to remedy this is to add some form of magnetic marker to the sample that can be seen with both SSM and XCT. For example, structured $\mathrm{Nb}$ can function as markers through the Meissner effect (visible in the SSM), while at the same time having a large density $\left(8570 \mathrm{~kg} \cdot \mathrm{m}^{-3}\right)$, making it easily discernible from magnetite in XCT. The only caveat with magnetic markers of any kind is that they will influence the magnetic field coming from nearby particles. This should be taken into consideration when designing the size and placement of such markers.

Because we used SSM for the magnetic imaging of the sample, the particles are cooled to $4 \mathrm{~K}$. This means the particles go through the Verwey transition of magnetite, a phase transition that includes a slight change in magnetic properties [74]. Below the transition temperature of $125 \mathrm{~K}$, the magnetisation decreases by about $15 \%$ [75]. This decrease is almost reversible and is not accompanied by a change in magnetisation direction [76].

Regardless, it would be better to avoid the Verwey transition altogether, meaning a room-temperature scanning magnetometry setup would be preferred. The large magnetic fields produced by the particles (typically $\pm 100 \mu \mathrm{T}$, see Figures 4.6 and 4.8) are several orders of magnitude above the noise limit of scanning SQUID sensors ( $50 \mathrm{nT}$ for our setup). Therefore, the high magnetic field resolution of a SQUID is not required to image these fields. Techniques such as scanning Hall probe microscopy, scanning nitrogen-vacancy-centre microscopy or scanning magnetic tunnel junction that can be operated at room temperature, which can achieve magnetic field resolutions of $10 \mu \mathrm{T}$ or better [77-79], might be better suited to this method. 


\subsection{Concluding remarks}

In this chapter, we used the SSM to image dipole-like magnetic objects and used fitting methods to extract the magnetic moments those objects. This enables us to learn more about the material itself (like $\mathrm{Fe}_{3} \mathrm{Se}_{4}$ in Section 4.3) or to use the information in other theories or models (such as the palaeofield model discussed in Section 4.4). The ability to quantitavely measure magnetic fields is a property that is sometimes lacking in other prevalent scanning magnetometry techniques, such as magnetic force microscopy. When combined with other techniques, such as XCT as discussed in Section 4.4, complicated magnetic structures can be inverted to obtain the magnetic moment.

For now, our fitting procedures assume the sensor is parallel to the $x y$ plane. As we established in Chapter 2, this is not the case for our setup, meaning we pick up some of the in-plane component of the magnetic field as well. While this does impact the inversion results, we estimate it to be less than $20 \%$ in a worst-case scenario based on additional simulation data (not shown here).

Although one can construct a model with all three field components for data fitting, this would increase the computational load of the inversion. While this might still be feasible for isolated objects that can be described as point-dipoles (like those in Section 4.3), for more complicated systems (like the clustered dipoles in Section 4.3 and the experiment in Section 4.4), where computation time is already the bottleneck, it becomes a more difficult balance between accuracy and time or resources invested. 


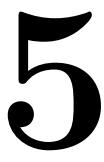

\section{Critical thickness behaviour in $\mathrm{LaMnO}_{3}$ thin films}

The perovskite-oxide $\mathrm{LaMnO}_{3}$ is an antiferromagnetic insulating material in the bulk. However, when deposited epitaxially on a $\mathrm{SrTiO}_{3}$ substrate, its ground state becomes ferromagnetic. Here, we will present work done to unveil the origin of this ferromagnetic ground state by means of several different characterisation methods, including synchrotron-based X-ray studies.

I would like to thank Prof. Dr. Mark Golden and Georgios Araizi Kanoutas, M.Sc. of the University of Amsterdam for the collaboration and for performing and analysing the synchrotron measurements. I would like to thank Dr. Nicolas Gauquelin of the University of Antwerp for the STEM-EELS measurements. Finally, I would like to thank André Timmermans, M.Sc. for fabricating some of the samples in this project. The contents of this chapter are currently being prepared for publication. 
A

$\mathrm{B}$

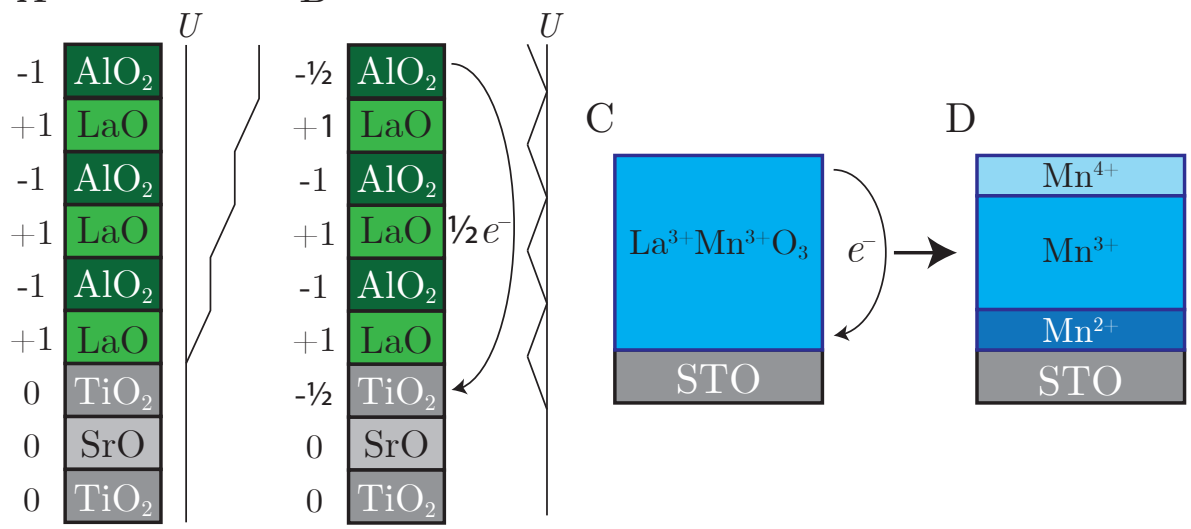

Figure 5.1: The polar catastrophe model. A) Increasing potential $U$ due to polar mismatch at the LAO-STO interface. B) Resolution of the diverging potential by transfer of half an electron from the surface to the $\mathrm{TiO}_{2}$ layer at the interface. A) Diverging potential causes migration of electrons from the LMO surface to the interface. B) Resulting LMO layered structure, with different valences at the surface, at the interface and in the film bulk.

\subsection{Introduction}

$\mathrm{LaMnO}_{3}$ (LMO) is a bulk antiferromagnetic insulator commonly known as the parent compound of colossal magnetoresistance materials such as $\mathrm{La}_{1-\mathrm{x}} \mathrm{Sr}_{\mathrm{x}} \mathrm{MnO}_{3}$ (LSMO) and $\mathrm{La}_{1-\mathrm{x}} \mathrm{Ca}_{\mathrm{x}} \mathrm{MnO}_{3}$ (LCMO) [80, 81]. However, when grown as a thin film on $\mathrm{SrTiO}_{3}(\mathrm{STO})$, LMO becomes ferromagnetic $[17,82-92]$. Recently, Wang et al. discovered that the ferromagnetic state depends on the thickness of the LMO layer [83]. Below 6 unit cells, the film showed no ferromagnetic behaviour as imaged by SSM, while films of 6 uc thick and above did.

As an explanation, Wang et al. offered a process similar to the one proposed for conductivity at the $\mathrm{LaAlO}_{3}-\mathrm{SrTiO}_{3}$ interface by Nakagawa et al. [93]. Due to a polarity mismatch at the LAO-STO interface, there is an internal potential in the LAO that increases with increasing thickness (Figure 5.1A). To compensate for this, electrons migrate from the surface to the interface, where they occupy Ti $3 d$ bands on the STO side of the interface, causing conduction (Figure 5.1B). This model is known as the 'polar catastrophe' model. 
Since LAO and LMO have the same alternatingly-charged sublayer structure, it seemed natural to adopt this hypothesis for the LMO-STO situation. In this case, the migrating electrons will occupy Mn $3 d$ states near the interface, since these have a lower energy compared to the $\mathrm{Ti}$ $3 d$ states in the STO $[88,94,95]$ (Figure 5.1C). Because of the electron transfer, local doping of the Mn then leads to ferromagnetism through the double exchange interaction [96-98].

If the ferromagnetic ground state is due to an internal redistribution of electrons as described above, three layers with distinct $\mathrm{Mn}$ valence states should be observable, shown in Figure 5.1D: an interfacial layer near the STO with a more $\mathrm{Mn}^{2+}$ character (due to the inclusion of migrated electrons); a 'bulk' layer with the expected $\mathrm{Mn}^{3+}$ character; and a surface layer which should have a more $\mathrm{Mn}^{4+}$ character due to the absence of the migrated electrons.

Alternative explanations for the ferromagnetic state have also been proposed. Calculations indicate that strain can play a significant role [99-104], and off-stoichiometry of the oxygen $[10,17,89-92,103]$ or either cation $[84,85]$ also influences the magnetic state. In this chapter, we will look at a suite of experiments designed to shed more light on the origin of the ferromagnetic state in LMO thin films. Using mostly synchrotronbased experiments, we will look at the valence state of the material as a whole as well as on a layer-by-layer basis.

\subsection{Sample fabrication}

The experiments described below were performed on several series of LMO thin films grown on both $\mathrm{STO}(001)$ and Nb-doped $\mathrm{STO}(001)$ substrates. $\mathrm{Nb}$-doped substrates were used in all synchrotron-based experiments in order to minimise charging effects. Other experiments made use of undoped STO substrates. Substrates were treated with buffered hydrogen fluoride to achieve $\mathrm{TiO}_{2}$-terminated surfaces and annealed at $950{ }^{\circ} \mathrm{C}$ for 1 hour to improve crystallinity [105].

The LMO layers were deposited using the pulsed laser deposition (PLD) method assisted with reflective high-energy electron diffraction (RHEED) in order to achieve monolayer control over the thickness. We used a laser energy of $1.3 \mathrm{~J} \cdot \mathrm{cm}^{-1}$ pulsed at $1 \mathrm{~Hz}$ in a $3 \times 10^{-2}$ mbar oxygen atmosphere with a substrate temperature of $750{ }^{\circ} \mathrm{C}$. After deposition, the sample was cooled to room temperature at $10^{\circ} \mathrm{C} \cdot \mathrm{min}^{-1}$ in the same 

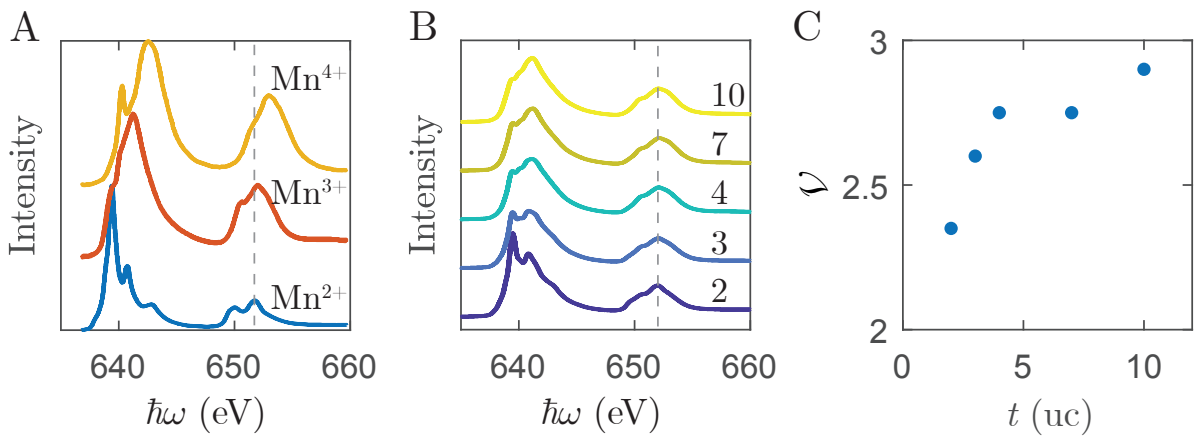

Figure 5.2: XAS measurements on the LMO $L_{2}$ and $L_{3}$ edge. A) Reference spectra used in the analysis, offset for clarity, from Reference [106]. The dashed line highlights the $L_{2}$ edge peak location for $\mathrm{Mn}^{2+}$. B) XAS measurements on LMO samples of different film thickness, offset for clarity. Labels indicate the LMO film thickness in uc. The dashed line highlights the $L_{2}$ edge peak location for $\mathrm{Mn}^{2+}$. C) Extracted average valence state of LMO films as function of thickness.

atmosphere.

\subsection{X-ray absorption spectroscopy}

X-ray absorption spectroscopy (XAS) is a way to probe the unoccupied energy levels of a material. An incident X-ray will be absorbed by electrons, which move from a core level into an unoccupied state. They can then relax by either emitting a photon or an electron. Since energy is conserved, only specific incident photon energies $\hbar \omega$ will cause photon or electron emission, depending on the electron energies of an atom. These energy levels are unique for each element, which means XAS is elementspecific. In addition, the valence state of the atom will also influence the exact values of the energy levels, meaning XAS can probe the electronic configuration of an atom in a material.

Figure 5.2 shows XAS measurements (performed at the 110 beamline of the Diamond Light Source in the United Kingdom) around the $L_{2}$ and $L_{3}$ edges of $\mathrm{Mn}$. The measurements were performed at $T=6 \mathrm{~K}$, well below the Curie temperature of LMO $(100-150 \mathrm{~K})$. The $L_{2}$ edge corresponds to $2 p$ electrons with $j=1 / 2, L_{3}$ to $2 p$ electrons with $j=3 / 2$. To extract the average valence state, we can fit these spectra using reference spectra, 
shown in Figure 5.2A [106]. The idea is that the weights used to fit these reference spectra to the data equal the ratio of valence states present. If we call the measured spectrum $f_{m}$, the reference spectra $f_{i}$ (with $i \in$ $[2+, 3+, 4+])$ and the corresponding weights $w_{i}$, then the average valence state $V$ can be determined:

$$
\begin{aligned}
f_{m} & =w_{2+} f_{2+}+w_{3+} f_{3+}+w_{4+} f_{4+}, \\
v & =\frac{2 w_{2+}+3 w_{3+}+4 w_{4+}}{w_{2+}+w_{3+}+w_{4+}} .
\end{aligned}
$$

Figure 5.2B shows the measured spectra on samples with different LMO thicknesses $t$. Comparing to the reference spectra, we can clearly see that with increasing thickness, the spectrum moves from dominantly $\mathrm{Mn}^{2+}$ like to dominantly $\mathrm{Mn}^{3+}$-like. Calculating the valence $\mathcal{V}$ and plotting it as a function of $t$ (Figure 5.2C), we can see that it increases from $2+$ to $3+$ with increasing thickness. $w_{4+}$ was equal to zero for all of these fits, implying that little-to-no $\mathrm{Mn}^{4+}$ is present in the films.

In addition, we can look at the location of the $L_{2}$ edge peak. In Figure 5.2A, we see that the peak shifts to higher energies for $\mathrm{Mn}^{4+}$, but its energy is approximately the same for the $\mathrm{Mn}^{2+}$ and $\mathrm{Mn}^{3+}$ spectra. When looking at Figure 5.2B, we find that the location of this peak does not move with increasing thickness. This again indicates that there is no $\mathrm{Mn}^{4+}$ present in any of our measured LMO films.

\subsection{Hard X-ray photoemission spectroscopy}

A second way to glean information on the valence state is to look at exchange splitting. The exchange interaction with the $3 d$-orbitals causes the $3 \mathrm{~s}$ core level to split. The magnitude of this splitting is directly related to the valence state of the $\mathrm{Mn}$ ion [107-110]. These states can be probed using hard X-ray photoemission spectroscopy (HAXPES), which was performed at the I09 beamline of the Diamond Light Source.

Figure 5.3A shows HAXPES data as a function of the binding energy $E_{b}$ around the Mn $M_{1}$ peaks, corresponding to $3 s$ electrons, taken at $T=250 \mathrm{~K}$. We can see that the exchange splitting $\Delta_{3 s}$ decreases with increasing $t$. From literature, we find that in a purely $\mathrm{Mn}^{2+}$ system, $\Delta_{3 s}=6.2 \mathrm{eV}$, while $\Delta_{3 s}=5.2 \mathrm{eV}$ for a $\mathrm{Mn}^{3+}$ system. The measured decrease in $\Delta_{3 s}$ with increasing thickness therefore implies a move from 

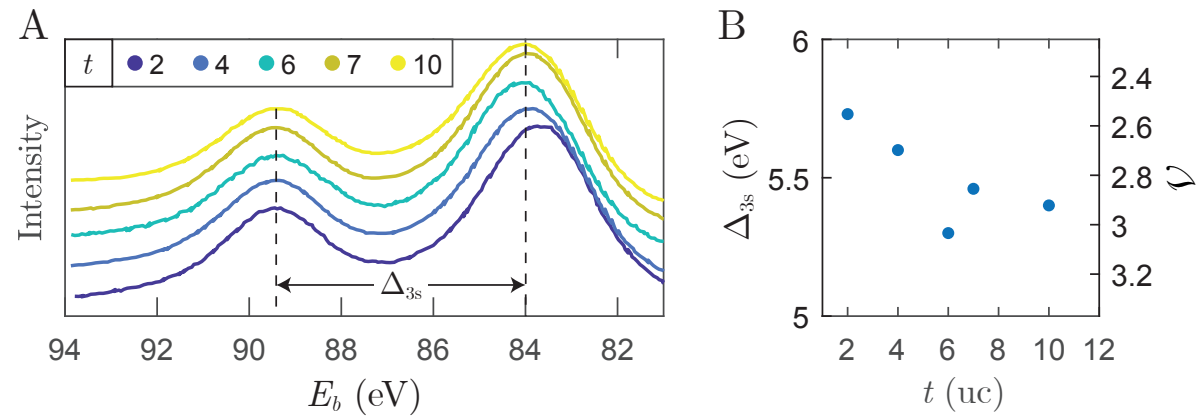

Figure 5.3: A) HAXPES measurements on the LMO $3 s$ state for different thicknesses, offset for clarity. B) Exchange splitting $\Delta_{3 s}$ as function of $t . \Delta_{3 s}$ can be converted to Mn valence, shown on the right $y$-axis (see Reference [107]).

more $\mathrm{Mn}^{2+}$-like to more $\mathrm{Mn}^{3+}$-like. This matches the results we found when measuring the Mn L-edge (Figure 5.2).

While the overall trend in Figure 5.3B tends towards a $3+$ character, the sample with $t=6$ uc shows a sudden increase in valence to about 3.05. This would indicate some $\mathrm{Mn}^{4+}$ is present in the film, even more than the amount of $\mathrm{Mn}^{2+}$. While the electronic reconstruction scenario has 6 uc as the critical thickness for electron transfer, and this transfer would create $\mathrm{Mn}^{4+}$ at the LMO surface, it creates an equal amount of $\mathrm{Mn}^{2+}$ at the interface and should thus not influence the total average Mn valence. We therefore conclude that the 6 uc sample is of such significantly different nature compared to the other samples (possibly related to issues during growth) that we will omit it from further analysis and discussion.

\subsection{X-ray magnetic circular dichroism}

$\mathrm{X}$-ray magnetic circular dichroism (XMCD) is an X-ray technique that is sensitive to the magnetic state of the material. By using either circular-left or circular-right polarised light, which are sensitive to different transitions depending on electron spin, the magnetic state of the material can be probed on an element-specific basis.

Figure 5.4 shows the XMCD data obtained at the I10 beamline of the Diamond Light Source. A typical measurement is shown in Figure 5.4A, in this case a 7 uc thick LMO film. We again scan across the $L_{2}$ and $L_{3}$ edges of Mn, giving us element-specific spectra similar to those in the 
A

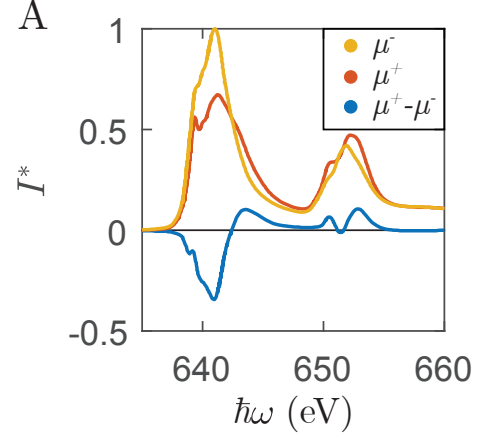

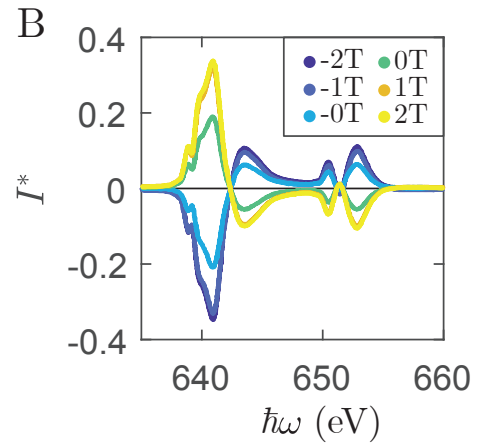

Figure 5.4: XMCD measurements on an LMO thin film with $t=7$ uc. $I^{*}$ is the signal intensity normalised to the $L_{3}$ edge maximum. A) Typical measurement at an applied field of $-2 \mathrm{~T}$, showing the circular-left $\left(\mu^{+}\right)$and circular-right $\left(\mu^{-}\right)$ polarised spectrum, as well as the difference between the two. B) XMCD signal at different applied fields. $-0 \mathrm{~T}$ indicates the field being swept to $0 \mathrm{~T}$ from negative field.

XAS measurements (Figure 5.2). We magnetise the sample by applying an external magnetic field along the in-plane crystal axes (the easy axes for LMO [83]). A clear difference is visible in the circular-left $\left(\mu^{+}\right)$and circular-right $\left(\mu^{-}\right)$polarised spectrum. This is indicative of spin alignment (e.g., there are more up-spins than down-spins). The difference between these two spectra (called the XMCD signal, also plotted in Figure 5.4A) therefore is clearly non-zero.

To be able to compare different XMCD signals, we normalise the measured intensity to the maximum of the $L_{3}$ peak of the spectrum $\left(\mu^{+}\right.$or $\mu^{-}$) with the highest $L_{3}$ peak signal (in the case of Figure $5.4 \mathrm{~A}, \mu^{-}$). This normalised intensity is labelled $I^{*}$.

However, a paramagnetic material would show a similar response to a ferromagnet under a constant applied field, since spins would also align in this case. By measuring at different fields, and specifically at zero field, we can distinguish between a paramagnet and a ferromagnet. After all, at zero field, only the ferromagnet should have a remanent magnetic moment (and therefore a non-zero XMCD signal); the paramagnet should not. As we can see in Figure 5.4B, we do indeed measure a non-zero XMCD signal indicative of ferromagnetism. We also find that the two measurements at zero field (one coming from negative field, one from positive) are of similar magnitude but with opposite sign. This is again what we would expect 
A

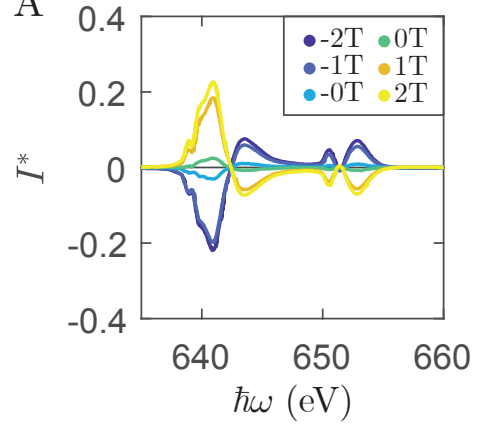

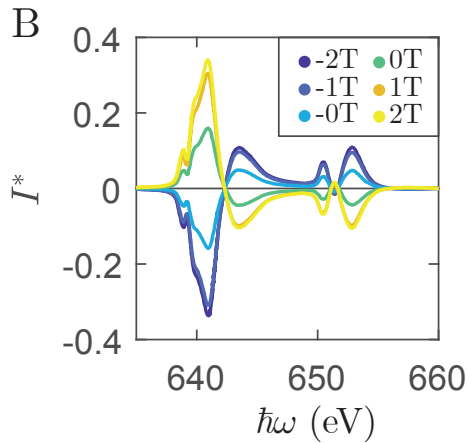

Figure 5.5: XMCD signal on an LMO thin film with A) $t=4$ uc and B) $t=10$ uc. $I^{*}$ is the signal intensity normalised to the $L_{3}$ edge maximum. $-0 \mathrm{~T}$ indicates the field being swept to $0 \mathrm{~T}$ from negative field.

of a ferromagnetic material, since the remanent magnetic moment will be aligned in the opposite direction.

Figure 5.5 shows further XMCD data on films with $t=4$ uc (Figure $5.5 \mathrm{~A}$ ) and $t=10 \mathrm{uc}$ (Figure 5.5B). As with the $7 \mathrm{uc}$ sample, both of these samples show a strong non-zero XMCD signal even at zero applied field, indicating ferromagnetic activity is present even below the reported critical thickness.

\subsection{Scanning transmission electron microscopy}

While the previous experiments gave us an idea of how the average valence state of Mn changes as the thickness increases, they do not give us any depth-dependent data. For this reason, scanning transmission electron microscopy (STEM) experiments were performed at the University of Antwerp to analyse the system on a layer-by-layer basis. By performing electron energy loss spectroscopy (EELS), we can attempt to measure the valence state of $\mathrm{Mn}$ in each individual layer, instead of only an average across the whole film. For this experiment, two LMO films were grown: one 3 uc thick, one 9 uc thick. Both of these films were then capped with 10 layers of LAO. This was done to protect the LMO layers during the STEM sample preparation process.

An elemental mapping of the location of different elements is shown in Figure 5.6A. While the interfaces are reasonably sharp, we can see that 
there is some diffusion of species across the interfaces. Most notably, there is a significant amount of La present in the STO substrate. However, since there is no clear indication of $\mathrm{Sr}$ on the LMO side of the interface, it does not appear to be an exchange of Sr and La across the interface between the two films. This excludes the possibility that the lower layers of LMO behave as LSMO, which is ferromagnetic.

Figure 5.6 shows the results of the EELS analysis on the two LMO films. We scanned the same energy range as in Figure 5.2, probing the Mn $L_{2}$ and $L_{3}$ edges. Similar to the analysis in Section 5.3, the spectra should relate to the valence state of $\mathrm{Mn}$. Looking at the first layer of both the 3 uc and 9 uc sample, we can discern a clear $\mathrm{Mn}^{2+}$ character. This further reduces the possibility of Sr doping in the lower layers, since the additional holes donated by $\mathrm{Sr}$ would lead to $\mathrm{Mn}^{4+}$. The remaining layers in both samples show a more $\mathrm{Mn}^{3+}$ character, indicating stoichiometric LMO in these layers. 

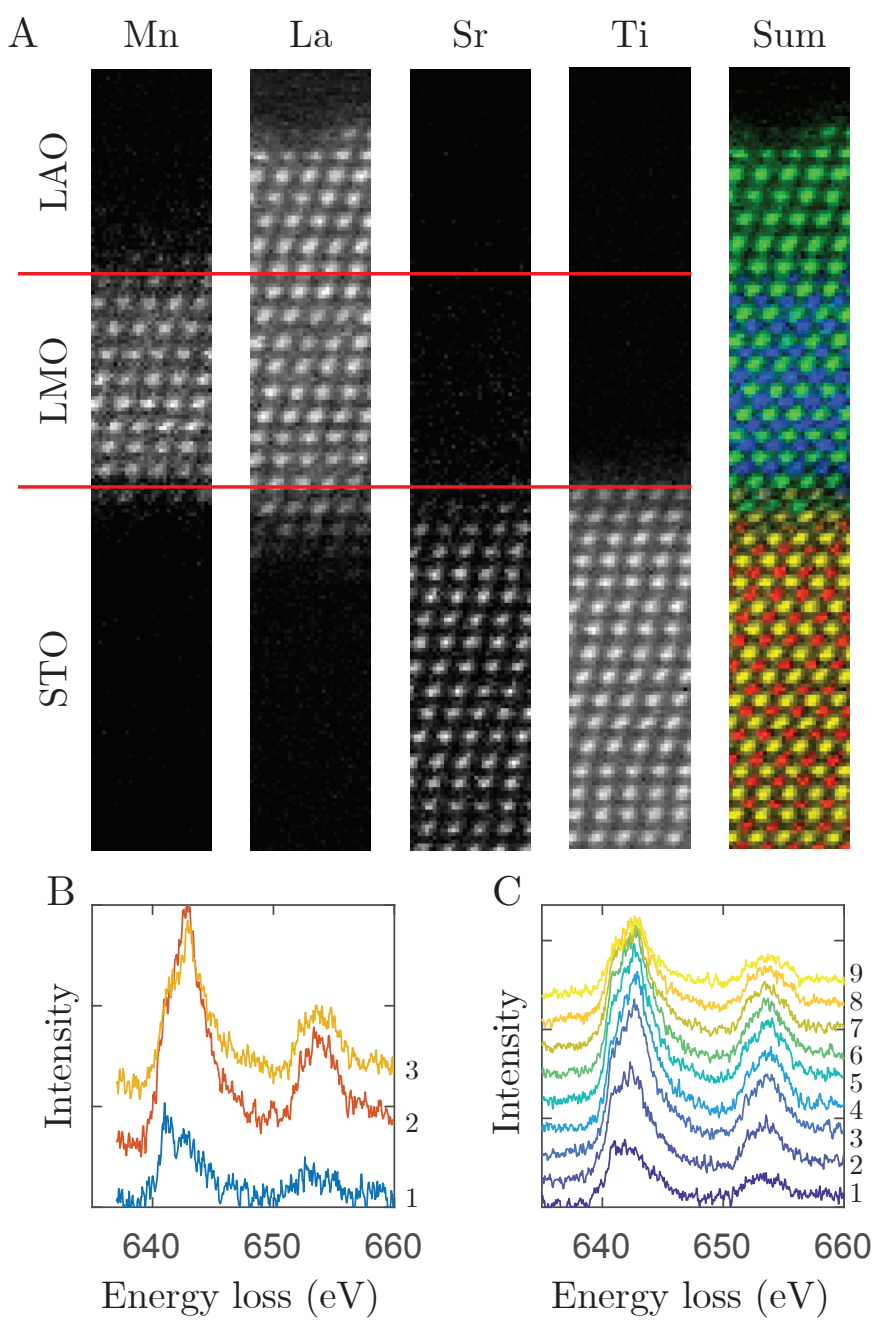

Figure 5.6: STEM experiments on two LMO films. A) Elemental mapping of Mn, La, Sr and Ti for a 9-uc-thick film. B-C) STEM-EELS analysis on LMO films of A) 3 uc thick and C) 9 uc thick. The labels indicate the LMO layer index, starting from the layer closest to the interface. Spectra are offset for clarity. 

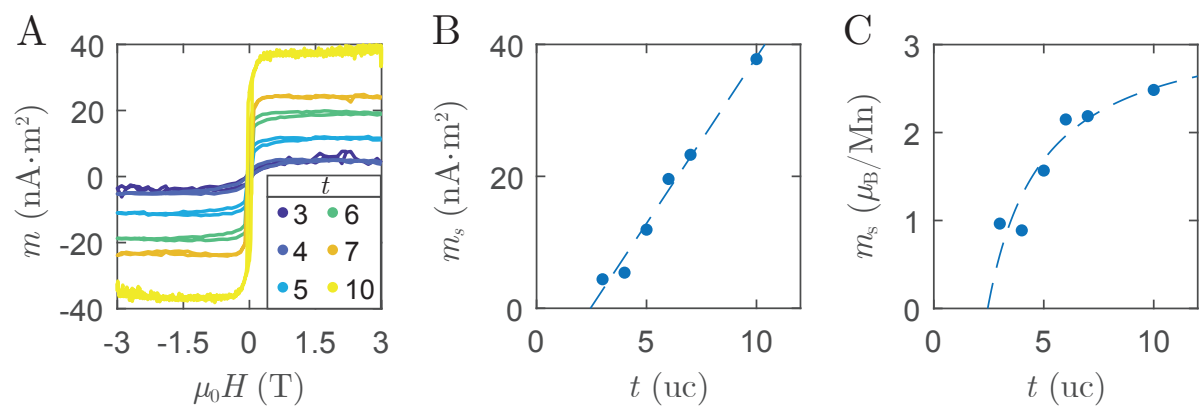

Figure 5.7: Vibrating sample magnetometry measurements on LMO films of varying thickness $t$. A) Magnetic moment $m$ as function of applied field for varying $t$. B) Saturation magnetic moment $m_{s}$ as function of $t$. The dashed line indicates a linear fit through the data. C) Saturation magnetic moment expressed as a number of $\mu_{B}$ per $\mathrm{Mn}$ ion as function of $t$. The dashed line is the same fit as in (B).

\subsection{Vibrating sample magnetometry}

To assess the average magnetic moment of the LMO films, we used vibrating sample magnetometry (VSM). Figure 5.7A shows the magnetic moment $m$ as a function of the applied field for different LMO film thicknesses, taken at $T=10 \mathrm{~K}$. For the analysis of these measurements, we have only subtracted a linear background to remove any diamagnetic response from the substrate. We can see that the film has a very small coercive field and reaches saturation around $0.5 \mathrm{~T}$.

In Figure 5.7B, we have extracted the saturation magnetic moment $m_{s}$ and plotted it as a function of $t$. We can see that $m_{s}$ increases linearly with increasing $t$ across the range between 3 and 10 uc. By extrapolating the curve down to $m_{s}=0$, we find that the first 2-3 layers appear to not be magnetically active. We also do not see a significant increase of the magnetic moment around $6 \mathrm{uc}$, the previously reported critical thickness.

Figure 5.7C shows the same data as Figure 5.7B but with the magnetic moment converted to a number of $\mu_{B}$ per $\mathrm{Mn}$ ion. This is calculated by taking $m_{s}$ and dividing it by the number of unit cells (= number of $\mathrm{Mn}$ ions). This is the ratio between the volume of one unit cell ( $\left.V_{\mathrm{uc}}=a \times b \times c\right)$, where $a$ and $b$ are in the in-plane lattice parameters and $c$ is the out-ofplane lattice parameter, and the total film volume $\left(V_{\text {film }}=L^{2} \times c \times t\right.$, with $L=5 \mathrm{~mm}$ the substrate side length). Assuming the film is fully strained 
to the substrate, $a=b=3.905 \AA$. Then:

$$
\begin{aligned}
& m_{\mu_{B} / \mathrm{Mn}}=\frac{m_{s}}{\mu_{B}} \frac{V_{\mathrm{uc}}}{V_{\mathrm{film}}}, \\
& m_{\mu_{B} / \mathrm{Mn}}=\frac{m_{s}}{\mu_{B}} \frac{a b}{L^{2} t} .
\end{aligned}
$$

Extrapolating the fit in Figure 5.7C, we find that each Mn ion contributes $3.3 \mu_{\mathrm{B}}$. This is less than expected from a $\mathrm{Mn}^{3+}$ ion, which would have a spin-only moment of $4.9 \mu_{\mathrm{B}}$ due to its 4 electrons [111]. It is also less than expected for either hole- or electron-doped $\mathrm{Mn}\left(3.9 \mu_{\mathrm{B}}\right.$ for $\mathrm{Mn}^{4+}, 5.9 \mu_{\mathrm{B}}$ for $\left.\mathrm{Mn}^{2+}\right)$, so it is not simply due to a mixture of $\mathrm{Mn}^{4+}$ and $\mathrm{Mn}^{3+}$. It likely indicates that not all $\mathrm{Mn}$ ions are coupled ferromagnetically. Assuming we only have $\mathrm{Mn}^{3+}$ ions, approximately $67 \%$ of $\mathrm{Mn}$ ions contribute to $m_{s}$.

\subsection{Scanning SQUID microscopy}

We used scanning SQUID microscopy to obtain additional information about the magnetic features of the LMO films. Figures 5.8A-E show typical SSM scans for LMO films of varying thickness between 4 and 10 uc. We can see that the magnetic field strength increases over several orders of magnitude with increasing thickness.

We also observe that for the 6 uc film (Figure 5.8C), the magnetic field profile looks very different from the other scans. From the image it would appear that the magnetic domains are highly oriented along the $x$-axis. Additionally, a vertically-oriented domain can be seen at the right edge. This vertical feature was present in every scan made on this sample (16 scans, each $250 \mu \mathrm{m} \times 250 \mu \mathrm{m}$ ), always at the right edge of the image. We therefore conclude that this is not a feature of the sample but indicative that there were issues with the SSM setup at that time, and so exclude this sample from further analysis and discussion.

We can alternatively display the increasing field strength by plotting $B_{\mathrm{RMS}}$ as a function of $t$, shown in Figure 5.8. As mentioned before, we can see $B_{\mathrm{RMS}}$ increasing over several orders of magnitude with increasing $t$. 
$\mathrm{A}$

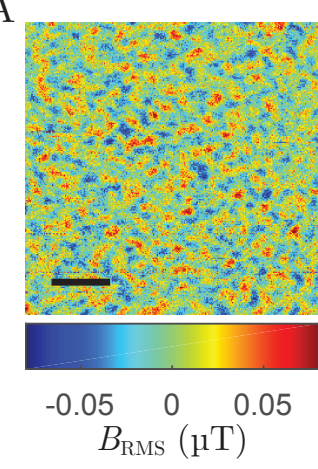

$\mathrm{D}$

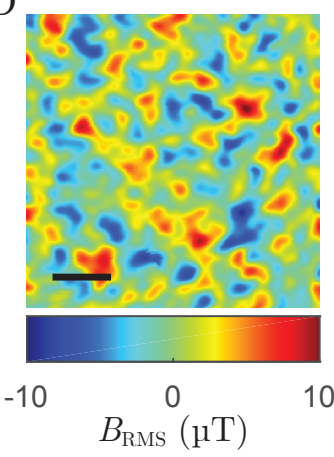

$\mathrm{B}$

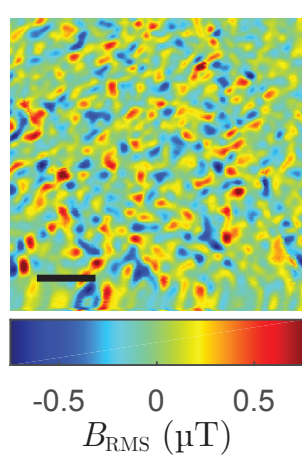

$\mathrm{E}$

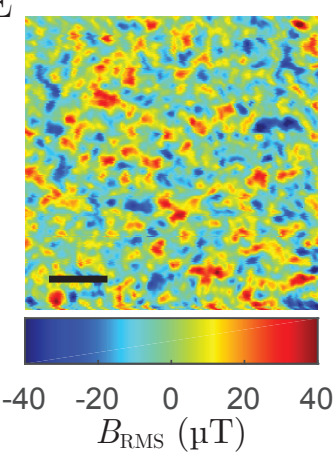

$\mathrm{C}$

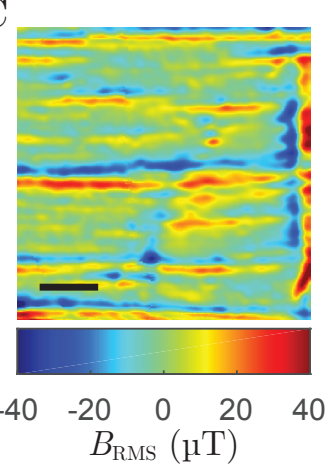

$\mathrm{F}$

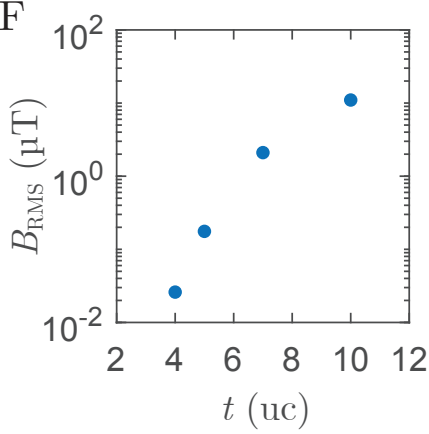

Figure 5.8: A-E) SSM images of LMO thin films of varying thickness: A) 4 uc; B) 5 uc; C) 6 uc; D) 7 uc; E) 10 uc. Scale bars indicate $50 \mu \mathrm{m}$. F) $B_{\text {RMS }}$ as a function of $t$.

\subsection{Discussion and conclusion}

In this chapter, we used various characterisation methods on ferromagnetic LMO thin films deposited on STO. The measurements were designed to yield more insight into the mechanism underlying the ferromagnetic ground state.

Using XAS measurements, we determined the average Mn valence state as a function of sample thickness. We did this by measuring the $L_{2}$ and $L_{3}$ edges (Figure 5.2). By fitting these to reference spectra, we found that the valence $V$ increases from a mixed $\mathrm{Mn}^{2+} / \mathrm{Mn}^{3+}$ state to a more pure $\mathrm{Mn}^{3+}$ state. Since the expected value for $\mathcal{V}$ should be $3+$, it is surprising we find a lower value for thinner films, down to $v=+2.4$ for $t=2 \mathrm{uc}$. This is most likely attributable to oxygen vacancies in the system which 
donate electrons.

As $t$ increases, we find that the average gets closer to $\mathrm{Mn}^{3+}$. In the polar catastrophe model (Figure 5.1), the average valence state of $\mathrm{Mn}$ remains $\mathrm{Mn}^{3+}$ since charge is neither lost nor gained, only redistributed (i.e., every $\mathrm{Mn}^{2+}$ at the interface has a matching $\mathrm{Mn}^{4+}$ near the surface). However, the lack of any $\mathrm{Mn}^{4+}$ contribution to the fits, as well as the lack of shift in the $L_{2}$ edge peak position (Figure 5.2B), indicates no presence of $\mathrm{Mn}^{4+}$.

Next, we look at the Mn $3 s$ states (Figure 5.3). Thinner films show a larger exchange splitting, meaning the average $\mathrm{Mn}$ valence is more $\mathrm{Mn}^{2+}$ like. For thicker films, it tends towards $\mathrm{Mn}^{3+}$ as we would expect. However, these measurements only show the total average valence state and not how electrons are distributed inside.

In Section 5.5 we showed the results of XMCD measurements, which allowed us to directly probe the magnetic character of the Mn in the LMO films. We saw that for all thicknesses measured, a non-zero XMCD signal was present at zero applied field, indicating some of the $\mathrm{Mn}$ is ferromagnetically coupled. The element-specific nature of the measurement shows directly that the ferromagnetism occurs in the LMO and not in the STO substrate. The results from the XMCD measurements can also be used to calculate the number of $\mu_{B} / \mathrm{Mn}$. This is done through a method known as the 'sum rules', which can yield both the orbital and spin components of the magnetic moment $[112,113]$. However, at the time of writing, this analysis was not yet finished.

By using STEM-EELS ${ }^{1}$ analysis (Figure 5.6), we find that even in thicker films, the interfacial layers keep their mixed $\mathrm{Mn}^{2+} / \mathrm{Mn}^{3+}$ character, while additional layers are mostly $\mathrm{Mn}^{3+}$. This means that even though the average tends towards $\mathrm{Mn}^{3+}$ for thicker films as shown in the XAS data, it is because of additional 3+-character layers and not because of a $\mathrm{Mn}^{4+}$ surface layer. The absence of this $\mathrm{Mn}^{4+}$ layer seems to indicate that a polar catastrophe is not responsible for a charge redistribution that leads to the ferromagnetic state.

This is corroborated by the VSM data in Figure 5.7. We see that the magnetic moment increases linearly for $t>4 \mathrm{uc}$. If a charge redistribution

\footnotetext{
${ }^{1}$ The STEM-EELS analysis involved samples that were capped with LAO in order to protect the LMO layers during processing. While the data we obtained was in line with the other experiments, it is still worthwhile to consider the possible influence of the LAO capping layer. We will look at this more closely in Chapter 6 .
} 
were to cause the ferromagnetic state, then only the top and bottom layers of the LMO film would contribute to the total magnetic moment. The bulk, 3+ layer should not have any contribution to the moment. This appears to be reflected in other publications as well $[87,90,95,114]$. In those reports, multiple groups report a number of $\mu_{B} / \mathrm{Mn}$ as a function of thickness. If an electronic reconstruction were the cause, then $\mu_{B} / \mathrm{Mn}$ should sharply increase around the transition but then decrease for increasing thickness, since any additional $\mathrm{Mn}^{3+}$ bulk layers do not contribute and thus the average number of $\mu_{B} / \mathrm{Mn}$. However, the above references report a constant or even increasing number of $\mu_{B} / \mathrm{Mn}$, contradicting the expectation of the model.

In the related LSMO and LCMO compounds, a magnetic 'dead layer' is often reported in thin film studies (for some examples, see References $[115-120])$. This is an interfacial layer that, though the material itself is ferromagnetic, shows no (or a significantly reduced) magnetic response. A consensus on the exact origin of the dead layer has not yet been reached, but oxygen vacancies appear to play an important role [120-122].

A similar LMO dead layer could form near the STO interface. We observed a mixed $\mathrm{Mn}^{2+} / \mathrm{Mn}^{3+}$ character near the interface, which is a consequence of oxygen vacancies in the system. These vacancies could disrupt the exchange interactions that cause LMO ferromagnetism by removing the intermediate oxygen atoms required. Several studies that changed the oxygen content of the LMO film do indeed show that lowering the oxygen content decreases the magnetic response [10,17,84-86, 90,123].

Although the oxygen vacancies might be a result of the sample fabrication process, it is also possible that they appear to compensate for the polarity mismatch $[103,124]$. The electrons created by the vacancies screen the internal potential and prevent it from diverging. In addition to these oxygen vacancies being detrimental to ferromagnetism, An et al. calculated that the breaking of the oxygen octahedra at the LMO surface also suppresses the ferromagnetic state [103].

Another possibility is related to the La inclusions in the STO seen in our STEM imaging in Figure 5.6. Substituting La for Sr would donate an electron to the system, which may migrate into the lower LMO layers and occupy Mn $e_{g}$ states, which lie lower in energy compared to the Ti $3 d$ levels $[94,125]$. This, then, might also explain the $\mathrm{Mn}^{2+}$ character of the LMO film near the STO interface.

Since several theoretical studies suggest that strain is what causes the 
ferromagnetic ground state, performing a strain-dependent study could shed more light on its role. While LMO has been grown on several different substrates with varying lattice parameters (see, for example, the supplementary info in Reference [83]), it is difficult to separate strain from other possible substrate influences, such as octahedral tilt, polarity or chemical interactions. A possible solution lies in a recent development in fabricating free-standing perovskite films [126-128]. One can imagine transferring a free-standing LMO film to a piezoelectric material in order to continually vary the strain along one or more axes and measure its magnetic activity as a function thereof.

If the critical thickness behaviour is merely the result of a magnetic dead layer at the interface and the magnetic moment increases linearly thereafter, this does not yet fully explain the observations made with SSM in Figure 5.8 and in Reference [83]. While the films below the critical thickness did show magnetic activity, the SSM signal (expressed as $B_{\mathrm{RMS}}$ ) increased by two orders of magnitude from 5 to 6 uc. If the magnetic moment increases linearly with thickness, then so should $B_{\mathrm{RMS}}$, as we have seen in Chapter 2 (Figure 2.11).

The answer might lie in Figures 2.11 and 2.12. It is possible that the magnetic domains in LMO thin films are the critical factor here. From those two figures, we can see that $\gamma$ (the conversion factor between magnetic moment and $B_{\mathrm{RMS}}$ ) can change considerably depending on the domain size and the sensor diameter. Figure 5.7A agrees in that it also shows a small domain size, indicated by the small coercive field. Furthermore, Anahory et al. also concluded that the ferromagnetism in LMO is due to small superparamagnetic domains in an otherwise antiferromagnetic matrix [87]. This means that even though the magnetic moment of the film as a whole increases linearly with thickness, changes in the domain size can lead to a change in $\gamma$ (and therefore, $B_{\mathrm{RMS}}$ ) which can be interpreted erroneously as a change in the magnetic moment. 


\section{6}

\section{Critical thickness behaviour in $\mathrm{LaMnO}_{3} / \mathrm{LaAlO}_{3}$ heterostructures}

LAO and LMO are perovskite materials that show remarkable changes in their properties when grown as a thin film on STO. These changes in properties are sometimes ascribed to the polar nature of these materials and its mismatch with the non-polar STO substrate. In this chapter, we will have a close look at the role of the polarity of these materials by combining them into heterostructures on STO. 


\subsection{Introduction}

Perovskite oxides are a class of materials with a large range of interesting properties such as high-temperature superconductivity, colossal magnetoresistance and multiferroicity [80,129-132]. In addition, due to their similar structure and lattice parameters, they can be fabricated into heterostructures with relative ease. This makes perovskites a promising platform for new technological applications.

Beyond the properties of the bulk materials, the interface between two perovskite oxides can also host properties not found in either compound. Most famously, the interface between the band insulators $\mathrm{SrTiO}_{3}$ and $\mathrm{LaAlO}_{3}$ (STO and LAO) gives rise to a two-dimensional conducting state [133]. Additionally, the conducting state only appears if the LAO layer is thicker than 4 monolayers [134]. The origin of this critical thickness behaviour is commonly attributed to an electronic reconstruction induced by the polarity-mismatch between LAO and STO [93] (see Chapter 5, Figure 5.1). However, this hypothesis is still under debate [124,135].

A second notable example is the antiferromagnetic LaMnO3 (LMO). When fabricated on top of an STO substrate, LMO thin films will show ferromagnetic behaviour [82], which has theoretically been explained as a strain effect $[103,104,136]$. Mirroring the LAO-STO situation, the polar LMO only exhibits the ferromagnetic state if the layer is sufficiently thick (i.e., > 6 uc) [83]. LMO shares several characteristics with LAO, such as its structure (with $\mathrm{Al}$ exchanged for $\mathrm{Mn}$ ) and the alternating charge of its sublayers. Therefore, the electronic reconstruction hypothesis was considered for this system as well [83]. Chapter 5 was devoted to investigating this hypothesis in more detail.

The proposed electronic reconstruction scenario is based on the alternating charge in the LAO layer [93]. The $\mathrm{La}^{3+} \mathrm{O}^{2-}$ sublayer has a net positive charge, while the $\mathrm{Al}^{3+} \mathrm{O}_{2}^{2-}$ sublayer has a net negative charge. When grown on the charge-neutral STO, the alternating charge causes an internal electric field and therefore a potential buildup (Figure 5.1A). Once the potential is high enough, electron transfer will occur from the surface to the interface, creating the 2-dimensional electron gas (2DEG, see Figure 5.1B). A similar argument can be made for LMO, which has a charged sublayer structure like LAO. Electrons migrate to Mn ions near the interface, causing a valency change and leading to double exchange interactions and ferromagnetism [103]. 
The question then arises: what if a secondary polar material is added to the first? For example, if a 3 uc LAO layer is deposited on STO, the interface is insulating. Then, if additional layers of a secondary polar material are added (e.g. LMO), the internal potential would continue to increase. Eventually, this could also lead to electron migration and the creation of the 2DEG. This way, a second polar material can be used to induce an electronic reconstruction in the first. Alternatively, the increased potential could cause the migration of electrons, but they stay within the LMO, leading to a ferromagnetic state instead.

How the situation is resolved depends on the exact details of the band structures involved. As the valence band is lifted up by the increasing potential, electrons will migrate to available states based on two criteria: the energy level of the state and the number of states available.

In this chapter, we will investigate the properties of combined LAO and LMO heterostructures of varying thicknesses and layer orderings. We will explore how the properties of these films change as a function of thickness and how this fits with current hypotheses.

\subsection{Fabrication}

The samples in this chapter are fabricated in a similar fashion to those in Chapter 5 (Section 5.2). All samples were deposited on $\mathrm{TiO}_{2}$-terminated STO(001) substrates using RHEED-assisted PLD. The LMO deposition parameters are the same as in Section 5.2. LAO was deposited at $850^{\circ} \mathrm{C}$ in a $1.8 \times 10^{-3}$ mbar oxygen atmosphere.

Samples were grown in two configurations: STO-LAO-LMO and STO-LMO-LAO. Unless otherwise noted, the second layer (i.e. capping layer) is always 10 uc thick, while the thickness $t$ of the primary layer is changed (Figure 6.1). To compare, three control samples were fabricated: 'LAO Control' (10

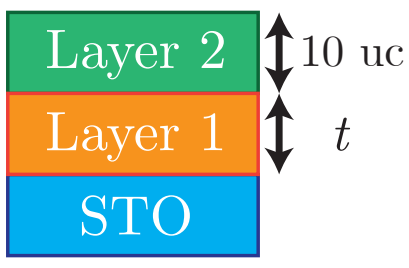

Figure 6.1: Schematic depiction of the sample layout. uc LAO only), 'LMO Control' (10 uc LMO only) and 'STO Control', which is a prepared substrate that has been exposed to deposition conditions (temperature and pressure) for 10 minutes. 


\subsection{STO-LAO-LMO}

In this section we will investigate how the properties of STO-LAO change upon addition of the LMO capping layer. Specifically, we are interested in the critical thickness of the LAO layer for conduction (4 uc), which one would naively expect to decrease upon the addition of more polar material. Secondly, we will also have a look at the ferromagnetic properties of these films. Kalisky et al. reported a critical thickness of $3 \mathrm{uc}$ for ferromagnetic behaviour to appear in STO-LAO systems [137].

\subsubsection{Transport measurements}

Transport measurements on the STO-LAO-LMO series were performed in a van der Pauw configuration; the results are shown in Figure 6.2. Figure $6.2 \mathrm{~A}$ shows the conductivity of the samples compared to LAO Control. As we can see, all our samples with an LAO thickness less than 4 uc are insulating, meaning the additional LMO layer has no influence on the critical thickness for conduction.

Although the 4 uc sample shows conductivity as expected, only the centre region of the sample is conducting. The outer $\sim 1 \mathrm{~mm}$ of the sample is insulating. This is largely due to two factors. First, the LAO thickness is very close to the transition point. Second, inhomogeneities inherent to the PLD process can cause the areas away from the centre to be slightly thinner than RHEED monitoring (aligned to the sample centre) indicates. These two factors can thus create insulating regions near the edges of the sample. Because of this, we will exclude this sample from the discussion below.

We find the conductivity to be constant as a function of $t$, in line with the early results by Thiel et al. [134]. Compared to the LAO Control sample, the conductivity is almost two orders of magnitude higher at $10 \mathrm{~K}$, indicating the LMO capping layer is having a significant influence on the conducting properties of our samples.

Figure $6.2 \mathrm{~B}$ shows the temperature dependence of the resistance of the conducting samples. We see that the capped samples have a higher resistance at room temperature, but a lower resistance at $10 \mathrm{~K}$ compared to LAO Control. Next, we extract the carrier mobility and density from Hall measurements using a two-band model (see the supplementary information in Reference [138]). Figures $6.2 \mathrm{C}$ and $6.2 \mathrm{D}$ show the obtained values for 

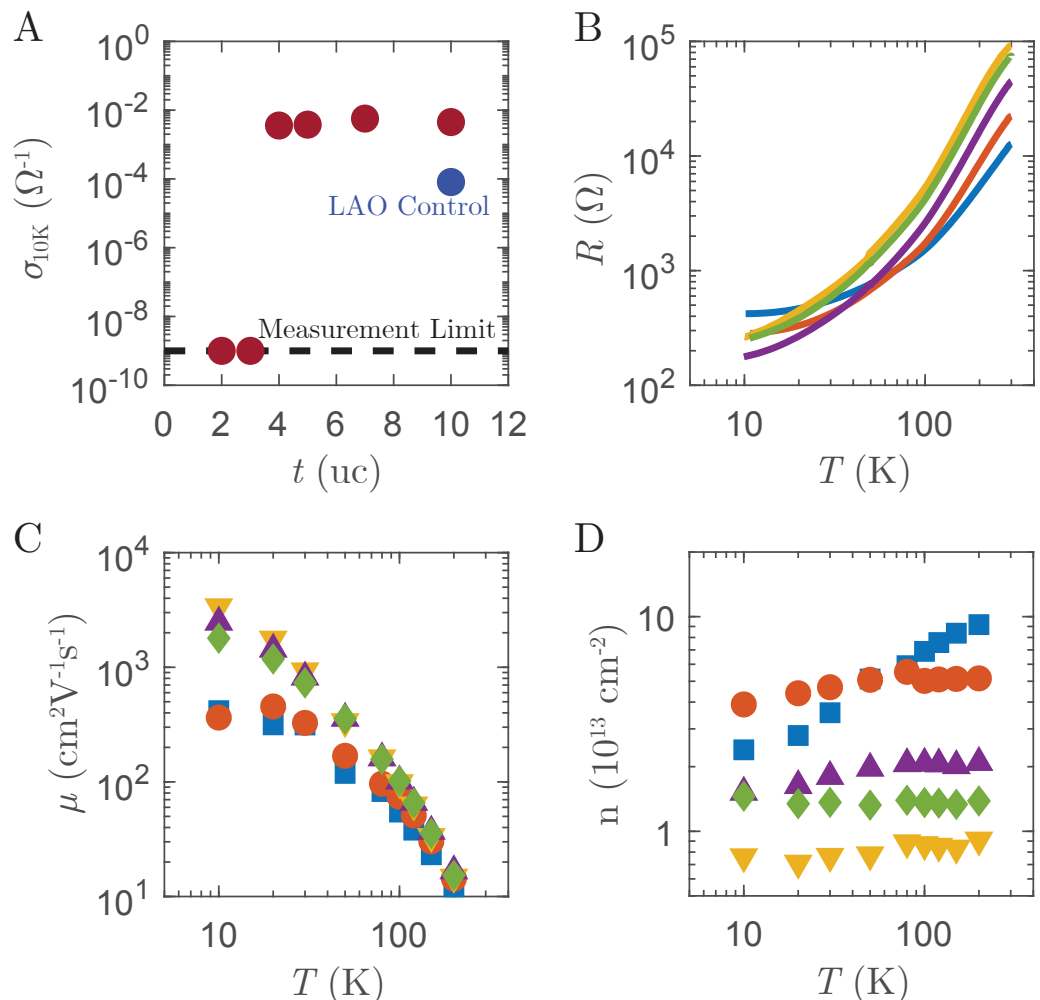

$\mathrm{D}$

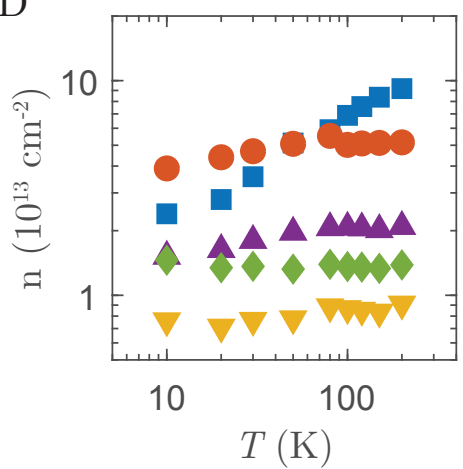

$t=4 \mathrm{uc} \quad \nabla t=5 \mathrm{uc} \quad \boldsymbol{\Delta} t=7 \mathrm{uc}$

$t=10 \mathrm{uc} \square \mathrm{LAO}$ Control

Figure 6.2: Transport properties of STO/LAO/LMO heterostructures. A) conductivity as function of the LAO layer thickness at $10 \mathrm{~K}$. B-D) Temperature dependence of the $(\mathrm{B})$ resistance $R,(\mathrm{C})$ majority carrier mobility $\mu$ and (D) majority carrier density $n$.

the mobility and density for the majority carriers. Figure $6.2 \mathrm{C}$ shows a clear increase in the mobility by almost an order of magnitude in the LMO-capped samples versus the uncapped control sample. Additionally, Figure 6.2D indicates a nearly temperature-independent carrier density in the capped samples, compared to the thermally activated carriers in the uncapped sample. The carrier freeze-out at low temperatures for LAO Control also explains the temperature behaviour of the resistance in Figure $6.2 \mathrm{~B}$. 

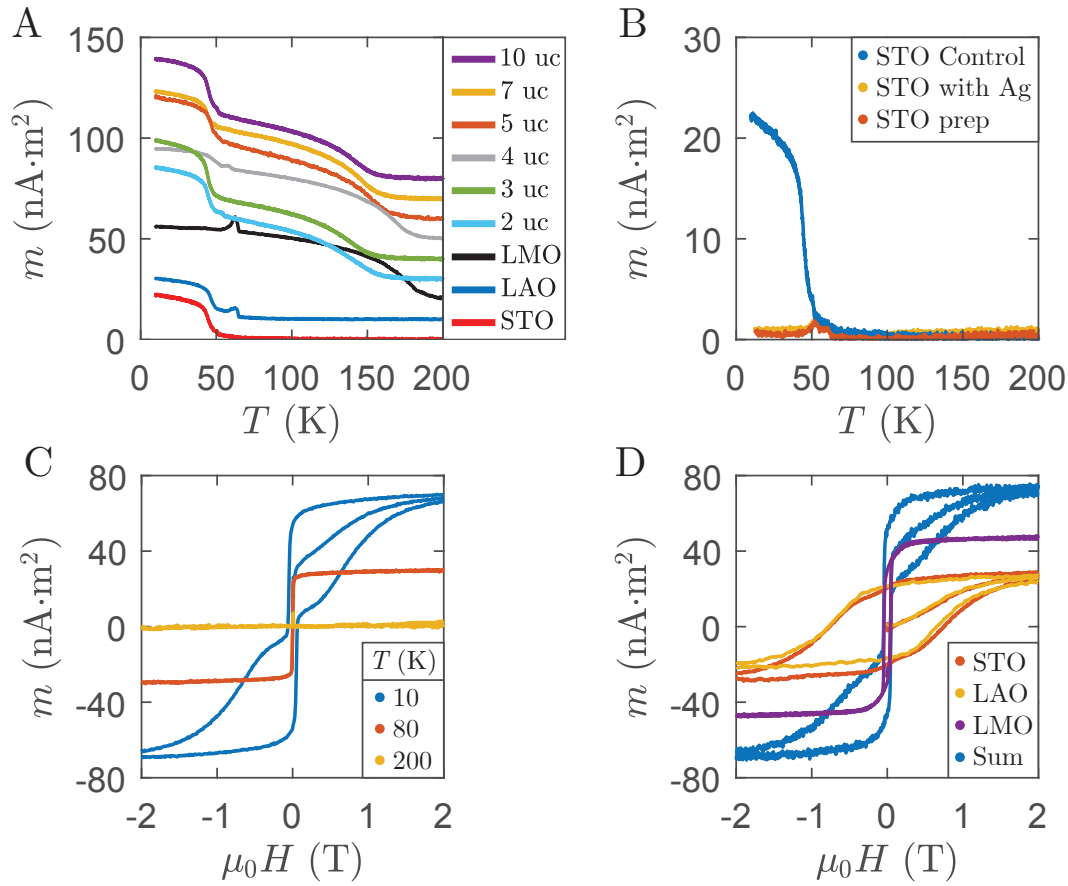

Figure 6.3: Vibrating sample magnetometry data of STO-LAO-LMO samples. A) Magnetic moment versus temperature, with the data offset for clarity. B) Magnetic moment versus temperature for STO substrates in different stages of preparation. C) Magnetic moment of the STO-LAO ${ }_{[10]}-\mathrm{LMO}_{[10]}$ samples versus applied magnetic field at different temperatures. D) Magnetic moment at $10 \mathrm{~K}$ versus applied magnetic field of the LAO, LMO and STO Control samples, as well as the sum of the LAO and LMO control samples.

\subsubsection{Magnetometry measurements}

Since the critical thickness for conduction does not change, it seems that the addition of the LMO layer does not lead to extra charge transfer to the STO interface. To investigate further, the magnetic properties of the heterostructures were measured with vibrating sample magnetometry (VSM) and are displayed in Figure 6.3. Figure 6.3A shows the magnetic moment of the STO-LAO-LMO series as a function of temperature, as well as the three control samples (LAO, LMO and STO). The samples were cooled from room temperature to $10 \mathrm{~K}$ in a $1 \mathrm{~T}$ field, and then measured while warming up to room temperature in a $0.1 \mathrm{~T}$ field. 
For the STO-LAO-LMO samples, two clear magnetic transitions are visible: one with a Curie temperature $T_{C} \approx 50 \mathrm{~K}$, and one with $T_{C} \approx$ $150-180 \mathrm{~K}$. Comparing these to the LMO Control sample, we see that it exhibits a slightly higher $T_{C}$ but a similar magnetic moment between $50 \mathrm{~K}$ and $150 \mathrm{~K}$. Therefore, in this region the magnetic moment can be attributed to the LMO capping layer. The peak that is seen in some samples between $50 \mathrm{~K}$ and $60 \mathrm{~K}$ is a measurement artefact inherent to our VSM setup.

The second transition, near $50 \mathrm{~K}$, also appears in the LAO and STO Control samples, indicating it is due to the STO substrate. Magnetic behaviour has been observed in LAO thin films on STO before [137,139,140], as well as in oxygen-deficient STO substrates [141]. DFT calculations also suggest that oxygen vacancies could cause ferromagnetism to appear in STO [142]. Since STO Control was exposed to low pressure, high temperature conditions, a significant amount of oxygen vacancies are likely present, which means they could be the origin of the observed ferromagnetic signal.

To confirm this hypothesis, we performed additional measurements on STO substrates at various points in the fabrication process. Three samples were measured: a substrate that was etched and annealed as normal (labelled 'STO prep'), a substrate with one surface covered in silver paint (used to attach the substrate to the PLD sample holder, labelled 'STO Ag'), and STO Control. The magnetometry data on these samples is shown in Figure 6.3B. We can see that only the STO Control sample shows a significant magnetic response, meaning we can rule out the substrate preparation process, the silver paint as well as general handling and storage of substrates as possible causes for the ferromagnetic character observed in STO Control.

Figure $6.3 \mathrm{C}$ shows the magnetic moment versus applied magnetic field for the STO-LAO ${ }_{[10]}-\mathrm{LMO}_{[10]}$ sample at three temperatures. At $200 \mathrm{~K}$, we observe no magnetic moment, in accordance with Figure 6.3A. The curve at $80 \mathrm{~K}$ shows only the component attributed to the LMO film, which is almost anhysteretic (similar to what we observed in Chapter 5, Figure 5.7). This could point to superparamagnetic behaviour, which has been observed in LMO before [87]. The $10 \mathrm{~K}$ curve shows the anhysteretic curve as well as a secondary component with a larger coercive field. Considering that this component only appears below $50 \mathrm{~K}$, it can be matched to the ferromagnetic signal from the STO in Figure 6.3A. 
To further illustrate this, Figure 6.3D shows hysteresis curves for the STO, LAO and LMO Control samples at $10 \mathrm{~K}$. For LMO, we again see nearly anhysteretic behaviour, similar to the $80 \mathrm{~K}$ curve in Figure 6.3C. The LAO and STO Controls show similar behaviour, indicating again that the ferromagnetism in the STO-LAO system is due to the STO substrate. If we sum the data of the LAO and LMO Control samples (labelled 'Sum' in Figure 6.3D), the result closely matches the $10 \mathrm{~K}$ measurement of Figure $6.3 \mathrm{C}$. This confirms that there are indeed two independent magnetic components present in the STO-LAO-LMO heterostructures.

\subsection{STO-LMO-LAO}

We now turn to samples with the reversed configuration: STO-LMOLAO. Here we will have a second look at the ferromagnetic behaviour as a function of thickness, following up on Chapter 5. There we concluded that the ferromagnetic state of LMO is likely a strain effect, and the critical thickness originally reported by Wang et al. (Reference [83]) is due to a magnetic dead layer near the interface as a result of oxygen vacancies.

As mentioned in Section 6.2, the thickness of the capping layer (now LAO) is kept fixed at $10 \mathrm{uc}$ while the LMO layer thickness is varied. We find all tested structures ( $3 \mathrm{uc} \leq t \leq 10 \mathrm{uc}$ ) to be insulating. This matches several findings in literature $[95,125]$. We therefore focus only on the magnetic properties of these films.

\subsubsection{Scanning SQUID microscopy}

Magnetism data was obtained using scanning SQUID microscopy (SSM). Figures $6.4 \mathrm{~A}$ and $\mathrm{B}$ show a comparison between two uncapped LMO films of 4 and 5 uc thick, respectively. There is a stark difference, with the signal increasing by over an order of magnitude by adding another layer of LMO. The result is similar to that found by Wang et al. [83], although the critical thickness now appears to be 5 uc instead of 6 uc. The difference might be because of the difference in deposition parameters.

Figure 6.4C shows SSM data for STO-LMO $[4]-\mathrm{LAO}_{[10]}$. We again see a sharp increase in the magnetic signal compared to Figure 6.4A. Since the LMO thickness is the same as the uncapped sample, the critical thickness has therefore decreased. This can be explained within the electronic reconstruction model since the potential would keep increasing in the LAO. 


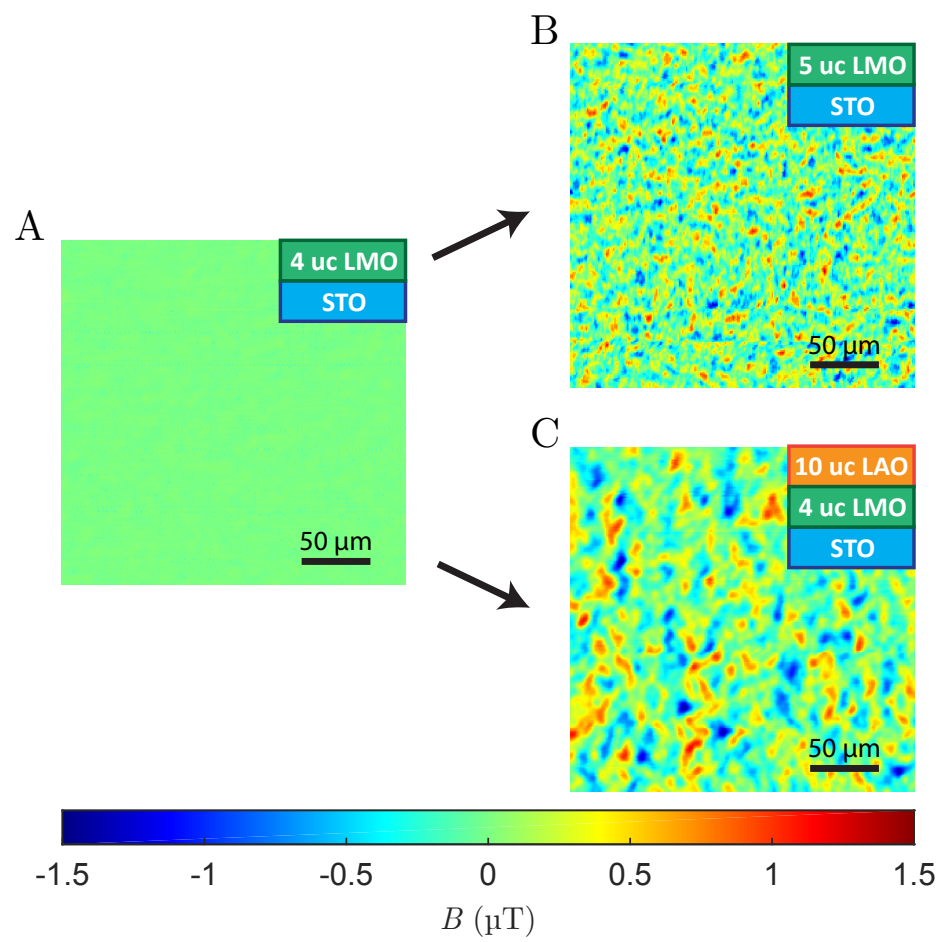

Figure 6.4: Scanning SQUID Microscopy data on capped and uncapped LMO samples. A) 4 uc LMO. B) 5 uc LMO. C) 4 uc LMO capped with LAO.

When electrons start migrating, they would move into the LMO $e_{g}$ states, which are lower in energy than available Ti $3 d$ states in the STO. The resulting mixed valence state in the LMO causes the ferromagnetic behaviour.

An alternative explanation would relate the strength of the ferromagnetic state to the number of oxygen vacancies in the LMO. An et al. recently calculated that oxygen vacancies are likely to form at the LMO surface, which suppress the ferromagnetic state by doping electrons into the system and creating magnetic dead layers near the interface. Additionally, the incomplete oxygen octahedra at the LMO surface have a similar suppressing effect [103]. It is possible that the addition of the LAO capping layer protects the LMO surface and prevents the formation of oxygen vacancies as well as completing the oxygen octahedra in the top LMO layer, thereby strengthening ferromagnetism in the film. 

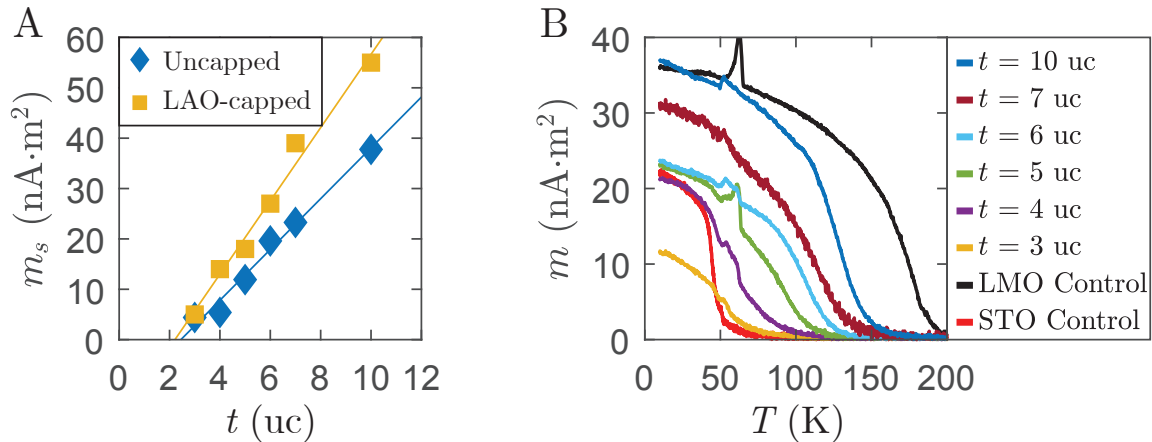

Figure 6.5: A) Saturation magnetic moment of STO-LMO-LAO and STO-LMO samples as a function of LMO layer thickness. The straight lines are linear leastsquares fits to the data. The uncapped LMO data is the same as in Chapter 5, Figure 5.7. B) Magnetic moment versus temperature for the STO-LMO-LAO series, compared to LMO and STO Control.

\subsubsection{Vibrating sample magnetometry}

Additional magnetometry data obtained with VSM is displayed in Figure 6.5. Figure 6.5A shows the saturation magnetization $m_{s}$ of the STO-LMOLAO samples, compared with uncapped LMO samples (from Chapter 5, Figure 5.7). A clear increase in $m_{s}$ for the capped samples is visible. A similar effect was recently described by Wu et al. [95]. This result matches the observation in the SSM measurements that the LAO cap strengthens the ferromagnetic state of the LMO film.

Interestingly, neither the capped nor the uncapped samples show signs of critical thickness behaviour such as we observed in the SSM data. For uncapped samples, the critical thickness should be around 5 uc as seen in Figure 6.4. However, both series show a critical thickness of approximately 2-2.5 uc, followed by a linear increase in total magnetic moment. This could indicate that the observation of a critical thickness in SSM data on LMO is not related to a sudden increase in magnetic moment.

The magnetization curves in Figure 6.5B for the STO-LMO-LAO samples only show a component related to the LMO layer. The magnetic signal coming from the STO substrate as seen before (Figure 6.3) is absent. This might be related to the filling of oxygen vacancies catalysed by LMO, which we will address in Section 6.5.

Next, by linear extrapolation we see that the magnetic signal only 
starts increasing after approximately 2-2.5 uc for both the capped and uncapped samples. This is again evidence of a magnetic dead layer at the interface between LMO and STO as discussed in Chapter 5. It appears that the LAO capping layer has no effect on the thickness of this dead layer, which is different from the results of $\mathrm{Wu}$ et al., who noted a decrease in the dead layer thickness upon capping with LAO [95].

\subsection{Discussion and conclusion}

In this chapter, we have looked at the influence of a polar capping material added to systems in which the interfacial polar mismatch is thought to play an important role in their properties. We started by looking at LAO on STO, capped with LMO. We found that the critical thickness for the 2DEG to appear, which is normally $4 \mathrm{uc}$, remained unchanged upon addition of the LMO (Figure 6.2A).

This is in contrast with e.g. STO-capping, which can reduce the critical thickness down to 1 uc [138], as can certain metals $[143,144]$, while LSMO capping can reduce the critical thickness to 3 uc [145]. Within the polar catastrophe scenario, the unaltered critical thickness despite additional polar layers might be due to the lower energy of the LMO $e_{g}$ bands with respect to the STO Ti $3 d$ bands $[94,125]$. This would cause any electrons that migrate due to potential build-up to stay within the LMO $[83,125]$.

We found that the addition of the LMO capping layer significantly increased carrier mobility (by about one order of magnitude) and removed thermally activated carriers from the system (Figures 6.2C-D). A study by Shi et al. investigated LAO films capped with Sr-doped $\mathrm{LaMnO}_{3}$ (LSMO). They noted a decrease in the carrier density upon capping, eventually turning the sample insulating at high (>7/8) Sr doping, though the carrier mobility remained unchanged [145]. They attributed the decrease in carrier density to a change in the formal polarization of the LSMO with increased doping. Since undoped LMO has the same polarization as LAO, this cannot be the explanation for the decreased density in our samples.

The changes in the mobility and carrier density are similar to those found in earlier studies on STO-LAO samples with an $\mathrm{SrCuO}_{2}$ (SCO) capping layer by Huijben et al. [146]. There, it was suggested that the changes were a result of the filling of oxygen vacancies catalysed by the SCO capping. We assume that LMO is playing a similar role in our samples, since LMO is a known oxygen-reduction catalyst [147]. Therefore, 
the oxygen defect states in STO, which give rise to thermally activated charge-doping, are removed. This might also explain why no ferromagnetic signal from the STO was present when LMO was the primary layer (Figure 6.5) versus when it was the capping layer (Figure 6.3).

We find no clear thickness-dependent behaviour in our samples (other than the conductivity increase for $t \geq 4 \mathrm{uc}$ ). Only Figure $6.2 \mathrm{C}$ seems to indicate a slight decrease in carrier mobility with increasing LAO layer thickness. One can imagine that, due to the increased layer thickness, the influence of the LMO capping layer decreases as it sits further away from the interface and therefore the 2DEG. However, this does not appear to be the case, since the overall conductivity and the carrier density appear not to follow a similar trend. The perceived trend in Figure $6.2 \mathrm{C}$ might therefore be due to sample-to-sample variations or an inaccuracy in the fitting procedure.

In the magnetometry measurements of the STO-LAO-LMO samples, we found two magnetic phases in the system: one from the STO substrate and one from the LMO capping layer (Figure 6.3). The magnetism originating from the STO substrate was attributed to the presence of oxygen vacancies $[141,142]$, which are created during the PLD process.

However, if these oxygen vacancies are partially removed upon capping with LMO (as we concluded from our transport measurements), then the electrons required for the ferromagnetism need to be sourced elsewhere. While the formation of the 2DEG could be a possibility, we see in Figure 6.3A that films below the critical thickness (2 and $3 \mathrm{uc}$ ) still show the STO being magnetically active without the presence of a 2DEG. Another option is La-substitutions in the STO during growth of the LAO and LMO. Our STEM images in Chapter 5, Figure 5.6A showed that La ions were present in the STO, which has been seen in other studies as well [148-150], though some experiments show no intermixing $[146,151]$.

The question then becomes why films with LMO as the first layer do not show any magnetic signal from the STO in Figures 5.7 and 6.5. One possible explanation here is that, if La-substitutions are the cause, the donated electrons likely migrate into the LMO and occupy available Mn $e_{g}$ states there $[94,125]$. We also mentioned this in Chapter 5 as a possible explanation for the formation of a magnetic dead layer.

In the second series of samples, with the STO-LMO-LAO configuration, we found all samples to be insulating. This is in line with other results in literature on LAO-capped LMO thin films [95, 125], as well as 
uncapped LMO. From our DFT calculations (not shown), we find that if electron transfer occurs, migrating electrons move into the LMO instead of the STO, preventing a 2DEG from forming.

Our SSM data in Figure 6.4 showed that adding the LAO capping layer significantly increased the stray field strength. Adding the LAO capping layer had a similar effect to adding an additional LMO monolayer and increased the magnetic field strength by an order of magnitude. While this could be indicative of a critical thickness effect, we concluded in Chapter 5 that a large portion of the perceived increase in $B_{\mathrm{RMS}}$ might be related to domain structure and how it affects SSM imaging, as discussed in Chapter 2. Indeed, we see in our VSM measurements (Figure 6.5) that while the addition of the LAO capping layer increases the magnetic moment for all tested thicknesses, the magnetic moment still increases linearly with thickness. From Chapter 2, we know that this should result in a linearly increasing $B_{\mathrm{RMS}}$ as well. Therefore, the discrepancy between the linearly increasing magnetic moment and non-linearly increasing $B_{\mathrm{RMS}}$ remains (as mentioned above, this is discussed in more detail in Chapter 5).

In this chapter, we attempted to influence the critical thickness behaviour of LAO and LMO layers on STO by adding additional polar layers. In the LAO case, the thickness required for the appearance of the 2DEG was not changed, which may be because of the lower-lying Mn $e_{g}$ orbitals being preferred by any migrating electrons over the Ti $3 d$ orbitals. Therefore, a different polar material, with energy levels such that migration to the STO is preferred, would be better suited to further investigate this concept.

Since the migration of electrons depends closely on the relative height of various energy levels, performing gating experiments can give additional insights into and possibly control over which levels electrons move into. The LAO-STO interface has shown to have manifold electric field effects when a top or back gate is added (for a selection, see References [152-155]). For our systems, which have a richer set of bands to play with, electric field experiments would surely be interesting. 


\section{Designing scanning SQUID experiments on topologically non-trivial materials}

In this chapter, we will look at topological materials and several aspects of these materials where scanning SQUID experiments might yield more insight. For each experiment, estimates of various material or setup parameters required for the experiment are given. Since some of the properties mentioned in this chapter, such as unconventional superconductivity, are not exclusive to topological materials, these proposed experiments can also be applied to other material systems.

I would like to thank Dr. Jorrit de Boer, Daan Wielens, M.Sc. and Liesbeth Mulder, M.Sc. for their contributions to this chapter. 


\subsection{Introduction}

In simple terms, topological insulators are a class of materials in which bands of opposite parity are inverted due to strong spin-orbit coupling. Upon contact with trivial matter (including the vacuum), these inverted bands must reconnect with their regular counterparts in order to preserve parity at the interface. This means that these bands must cross the Fermi energy, creating conducting states at the boundaries of the topological insulator.

In a mathematical sense, the number of inversions can be represented by a topological invariant. A change of this invariant requires a closing of the band gap. Therefore, interfacing a topological insulator with a regular material (which has a different topological invariant) must lead to a closed band gap at that interface. This, then, is what causes conducting states to appear.

This chapter will highlight several interesting properties of topological materials, specifically those where an SSM setup could contribute experimentally. In some cases, basic estimates will be given in order to see in which parameter space (that of either the material or the measurement setup) the experiments would need to be performed.

\subsection{Edge currents}

In a 3D topological insulator (TI), the band crossings at the interface cause the (2D) surfaces to become conducting. If we reduce the material thickness and move from a $3 \mathrm{D}$ to a $2 \mathrm{D}$ topological insulator, the $2 \mathrm{D}$ conducting surfaces will transform into 1D conducting edges. If a current is applied to a 2D topological material, the current paths along these edges will create a magnetic field. If these states are truly $1 \mathrm{D}$, then we can model this magnetic field like one coming from a wire, similar to what we did in Chapter 2.

Figure 7.1 shows a comparison between a $20 \mu \mathrm{m}$-wide homogeneous conducting sheet and a 2D TI of the same width. Both are carrying a current of $1 \mu \mathrm{A}$, and the profile is simulated for $h=5 \mu \mathrm{m}$. Figure 7.1A and B show a simulated SSM image of the sheet and TI, respectively. There is a clear visible difference in the profiles of the two conductors: the TI shows a positive and negative peak near each edge, whereas the conducting sheet only shows either a positive or negative peak at each 
A

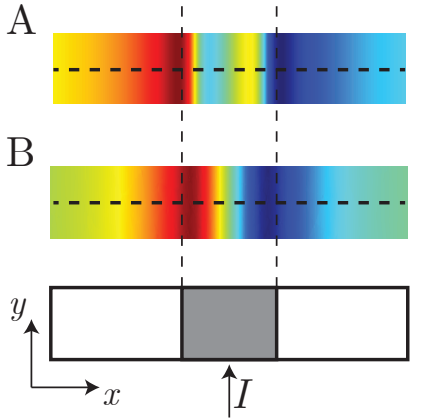

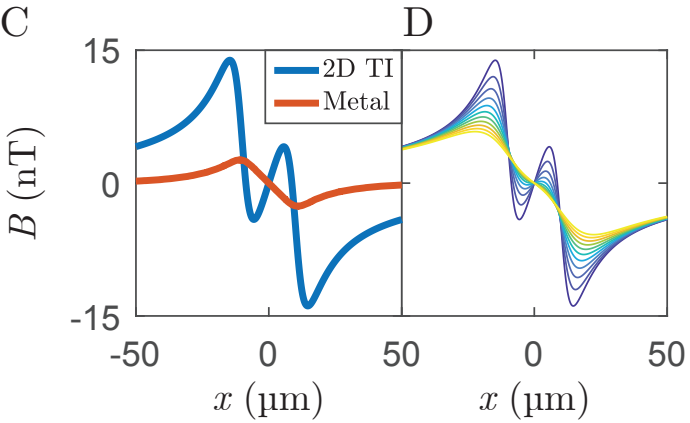

Figure 7.1: Simulated magnetic field profile above a current-carrying A) 2D topological insulator and B) trivial conductor. C) Line profile as indicated by the dashed lines in (A) and (B). D) Line profile as indicated by the dashed line in (A) for different values of $h$.

edge. The line profiles in Figure 7.1C further highlight this difference.

The visibility of the two separate edge currents depends on the resolution of the system. As discussed in Chapter 2, this depends on the height $h$ and the pickup loop diameter $d$ of the SSM. Figure 7.1D shows the same line profile as in Figure 7.1C for different values of $h$. We can see a transition from both current paths individually (i.e., one positive and negative peak at each edge) to a profile similar to that of the current sheet. If we take the transition point to be the point where the derivative at $x=0$ becomes zero, then the transition point is $h=\frac{w}{2}$. Therefore, as long as $w \gg h$, the two edge states should be clearly discernible.

The above holds for an ideal, single-crystal slab of TI. Grown thin films will usually have a more granular structure. Instead of flowing at the edge of the fabricated structure, current will run along grain boundaries, which form local material edges. Because of this, the current through the material and, therefore, the magnetic field produced by it will form a complicated web of current paths. One can imagine that an SSM image of such a current distribution might look similar to that of a ferromagnetic domain structure (e.g., Figure 2.10), though of course the origin is very different. With proper field and spatial resolution, the magnetic field data can be inverted to obtain the current distribution [40]. 


\subsection{Spin-momentum locking}

The strong spin-orbit coupling that causes the topological state in these materials has a secondary effect: spin-momentum locking. In simple terms, it means that charge carriers travelling in a certain direction will have one specific spin state. This effect strongly suppresses scattering, since a scattering event must come with a change of spin. This is especially relevant for the $1 \mathrm{D}$ edge channels in a $2 \mathrm{D}$ topological insulator, since the spins associated with the two allowed momentum states (i.e., forwards and backwards) are orthogonal.

However, spin-momentum locking means that an applied current will consist of charge carriers with identical spin. Moreover, while any current will produce a magnetic field circulating the current vector, such a spinpolarised current will produce a secondary magnetic field because of its net spin. To prove this, one can attempt to measure the magnetic field produced by a spin-polarised current and divide it into the spin component and the current component.

To model the above, we use the regular formula for the magnetic field $B_{\text {I }}$ produced by a current. To that, we add the magnetic field $B_{s}$ produced by the net spin polarisation of the current. For this component, we need to know the number of electrons $n$ involved in transport. For a 1D channel, we can write [156]:

$$
I=n e\langle v\rangle,
$$

where $\langle v\rangle$ is the average velocity of the charge carriers. Looking at the Dirac cone for such a system (Figure 7.2A), we find that $n$ equals the difference $\Delta n$ between the electrons at positive $k$ and those at negative $k$ (sometimes referred to as left-movers and right-movers). This also means that these electrons all move at the Fermi velocity $v_{F}$, so we get

$$
\Delta n=\frac{I}{e v_{F}} .
$$

The spin-component of the magnetic field $B_{\mathrm{s}}$ is given by integrating the magnetic moments along the current line. If we assume this line to be in the $y$-direction at $x=0$, then we get

$$
B_{s}=\frac{\mu_{0} \mu_{B} \Delta n}{2 \pi}\left[\frac{2 u_{x} h x}{\left(x^{2}+h^{2}\right)^{2}}-\frac{u_{z}\left(x^{2}-h^{2}\right)}{\left(x^{2}+h^{2}\right)^{2}}\right]
$$


A

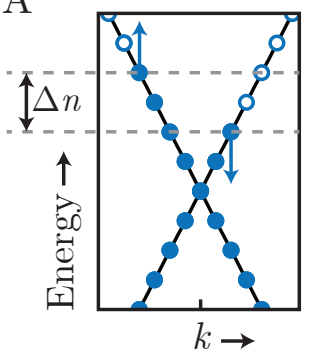

Filled oEmpty
$\mathrm{B}$

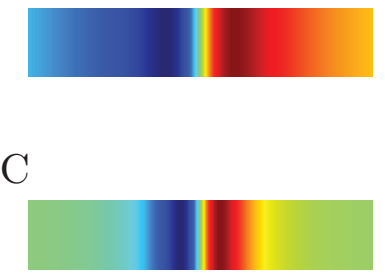

$\mathrm{D}$

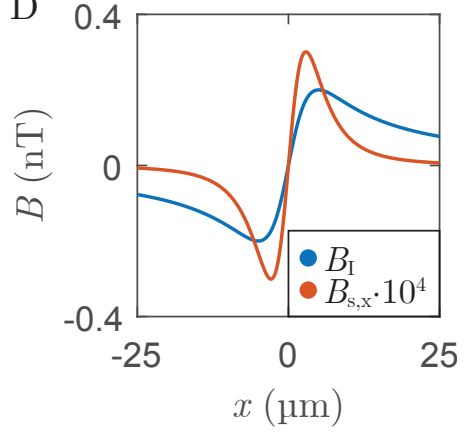

Figure 7.2: A) Schematic of a Dirac cone in an applied electric field along the positive $k$-direction. Arrows indicate the spin direction of electrons on that branch. B-C) Simulated magnetic field profile for B) $B_{\mathrm{I}}$ and C) $B_{\mathrm{s}, x}$. D) Line profiles from (A-C).

where $\hat{u}=\left\{u_{x}, u_{y}, u_{z}\right\}$ is the unit vector describing the orientation of the spins. The component belonging to $u_{y}$ is zero because aligning along the direction of the current line makes the magnetic field of each electron cancel out with that of the next. Only for a current line of finite length will the $u_{y}$ component show a non-zero contribution when the magnetic field at either end of the conductor is no longer compensated. Additionally, the spin-momentum locking is such that the spin will be aligned in the $x y$-plane. Therefore, $u_{z}$ will be zero.

Figure 7.2 shows the results of these calculations, assuming $v_{F}=10^{5}$ $\mathrm{m} \cdot \mathrm{s}^{-1}$. Figure 7.2B shows $B_{\mathrm{I}}$, while Figure 7.2C shows $B_{\mathrm{s}}$ for $\hat{u}=\{1,0,0\}$ $\left(B_{\mathrm{s}, x}\right)$. Comparing the different profiles in Figure 7.2D, we see that the $B_{\mathrm{s}}$ signal is roughly four orders of magnitude smaller than that of $B_{\mathrm{I}}$, on the order of $10 \mathrm{fT}$. This means it would be practically impossible to measure.

When studying this system, we have two variables to adjust that will change the relative signal strength of $B_{\mathrm{I}}$ and $B_{\mathrm{s}}: v_{F}$ and $h$. We can define a ratio $B^{*}$, which is the ratio between the maximum field strength of $B_{\mathrm{I}}$ and $B_{\mathrm{s}}$ and is given by

$$
\begin{aligned}
B^{*} & =\frac{25 e}{27 \mu_{B}} v_{F} h \\
& \approx 1.6 \cdot 10^{4} v_{F} h .
\end{aligned}
$$

This means that ideally we want $v_{F} h \approx 10^{-4} \mathrm{~m}^{2} \cdot \mathrm{s}^{-1}$ in order to have sim- 
ilar signal strength of $B_{\mathrm{I}}$ and $B_{\mathrm{s}}$. For a height of roughly $50 \mathrm{~nm}$ attainable by some SSM setups $[157,158]$, this would require $v_{F} \approx 2 \times 10^{3} \mathrm{~m} \cdot \mathrm{s}^{-1}$, far lower than typical values found in, for example, Bi-derived topological insulators [159]. Therefore, even with an optimised value of $v_{F} h, B^{*}$ might still be on the order of 10-100, making such an experiment and the analysis thereof difficult, though not necessarily impossible.

\section{$7.4 \quad p$-wave superconductivity}

We already encountered unconventional superconductivity in Chapter 3, where we studied structures containing the $d$-wave superconductor YBCO. Both $s$-wave and $d$-wave pairing symmetries are spin-singlet symmetries with an even orbital component. However, spin-triplet pairing is also mathematically possible, which will have an odd orbital component. The most commonly discussed among of is $p$-wave symmetry.

$\mathrm{Sr}_{2} \mathrm{RuO}_{4}$ is the poster child for possible $p$-wave superconductivity. Several experiments have provided strong indications of its unconventional superconducting nature, though not all results match up with the predicted behaviour of such systems $[160,161]$. Topologically non-trivial matter is a prime candidate for $p$-wave superconductivity. While this can be induced via the proximity effect, some topological insulators can become superconducting on their own [162].

\subsection{1 $p_{x}$-type symmetry}

Several variants of $p$-wave symmetry are predicted to exist. The basic forms are the $p_{x}, p_{y}$ and $p_{z}$ symmetries, which we will collectively call $p_{x^{-}}$ type. Figure 7.3 shows the previously discussed $s$ and $d_{x^{2}-y^{2}}$ symmetries alongside the $p_{x}$ symmetry. Like $d$-wave pairing, $p_{x}$-type pairing also has nodes (in this case, the $y z$-plane). In addition, the phase of the wave function changes by $\pi$ when going from $k$ to $-k$.

Based on this, one may quickly envision a set of experiments akin to those we performed in Chapter 3. The simplest would be to fabricate a series of junctions along different angles to the material's crystal axes. As with the YBCO junctions in Chapter 3, Sections 3.4 and 3.6.1, the different junctions should show differing characteristics since the gap size is angle-dependent. 
A

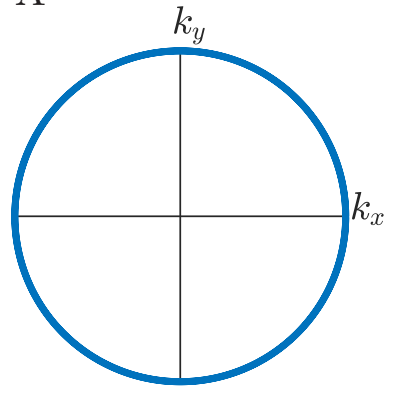

$\mathrm{B}$

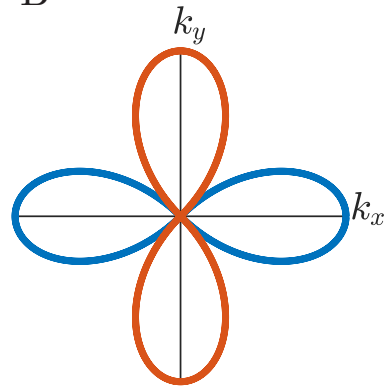

C

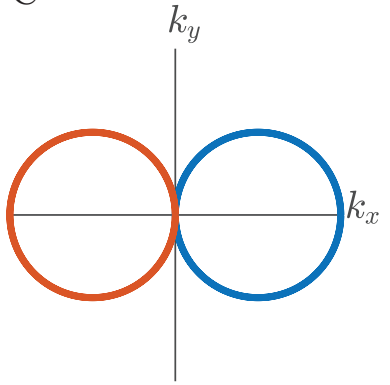

Figure 7.3: Symmetry and phase of the order parameter in A) $s$-wave, B)

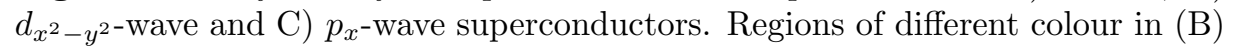
and $(\mathrm{C})$ have a $\pi$ phase-shift between them.

From there, we can look at such a superconductor as part of a SQUID. Since the phase is not constant for $p$-wave symmetries, one can fabricate SQUIDs that exploit the geometry to pick up additional phase jumps. In the case of $p_{x}$, we obtain a $\pi$ phase shift when going from $+k_{x}$ to $-k_{x}$. Therefore, fabricating a SQUID that is half $s$-wave, half $p$-wave would create a $\pi$-loop (Figure 7.4A).

Whether or not the structure is indeed a $\pi$-loop can then be verified in two ways. We can study the dependence of the critical current $I_{c}$ on the applied magnetic flux $\Phi_{e}$, which should have a peak at $\Phi=0$ if the symmetry is of a $p_{x}$-type. The second option is to see if spontaneous magnetic flux is generated, which can be done with any magnetically sensitive scanning technique. In addition, we can verify that this spontaneous flux equals $\frac{1}{2} \Phi_{0}$, for which SSM is particularly useful.

Doing both types of measurements on the same device is possible, though we need to keep in mind that the inductance can play an important role. Specifically, a high inductance would be advantageous when measuring the spontaneous flux and the value thereof (see Chapter 3, Figure 3.3) but also reduces the modulation depth of the SQUID when looking at $I_{c}\left(\Phi_{e}\right)$. Ideally, one would fabricate two devices, one for each experiment. However, in cases where this is not feasible (for example, when devices are fabricated out of exfoliated flakes of material), a compromise for the inductance must be found.

When verifying the $d$-wave nature of YBCO, Kirtley et al. used annular structures that were part $\mathrm{YBCO}$, part $\mathrm{Nb}$. By varying the proportion 
A

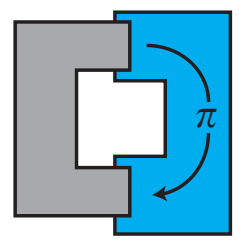

B

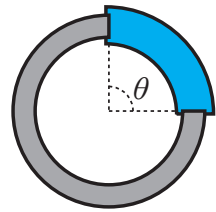

$\mathrm{C}$

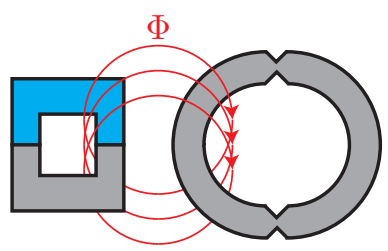

Conventional superconductor

Unconventional superconductor

Figure 7.4: Schematic layouts for probing order parameter symmetry. A) SQUID with a $\pi$ phase shift. B) Angle-resolved variant of (A). C) Measuring the magnetic flux state of (A) using an on-chip SQUID device.

of the ring made out of YBCO (given by an angle $\theta$; see Figure 7.4B), they were able to nicely show the in-plane pairing symmetry [50]. Of course, this design can be used to test for any symmetry, as long as the material to be studied can be fabricated into such rings.

\subsection{2 $p_{x} \pm i p_{y}$ symmetry}

Because of the strong spin-orbit coupling, topological superconductivity likely comes in the form of $p_{x} \pm i p_{y}$ pairing. This symmetry is gapless for all $\left(k_{x}, k_{y}\right)$, as opposed to symmetries of $p_{x^{-}}$type or $d_{x^{2}-y^{2}}$ discussed before. Additionally, the phase now linearly increases with increasing angle, unlike the step-wise increases we studied before. Due to its finite angular momentum, $p_{x} \pm i p_{y}$ pairing has a chirality which determines in which direction (clockwise or anticlockwise) the phase winds. For this reason, this pairing symmetry is also known as chiral $p$-wave.

Looking back at Figures $7.4 \mathrm{~A}$ and $\mathrm{B}$, using an angle $\theta=90^{\circ}$ and inducing a $\pi / 2$ phase shift could lead to a spontaneous fractional flux of $\pm \frac{1}{4} \Phi_{0}$, depending on the chirality of the unconventional superconductor. Using Equation 3.1, but replacing the $\pi$ phase shift with $\pm \pi / 2$, and again taking the limit $L \rightarrow \infty$ (meaning $\varphi_{1}, \varphi_{2} \rightarrow 0$ ), we find

$$
\begin{aligned}
2 \pi n & =\frac{2 \pi \Phi}{\Phi_{0}}+\varphi_{1}+\varphi_{2}+ \pm \frac{\pi}{2}, \\
\Phi & =\left(n \mp \frac{1}{4}\right) \Phi_{0} .
\end{aligned}
$$

Since chiral $p$-wave is degenerate (i.e., $p_{x}+i p_{y}$ and $p_{x}-i p_{y}$ have the same 

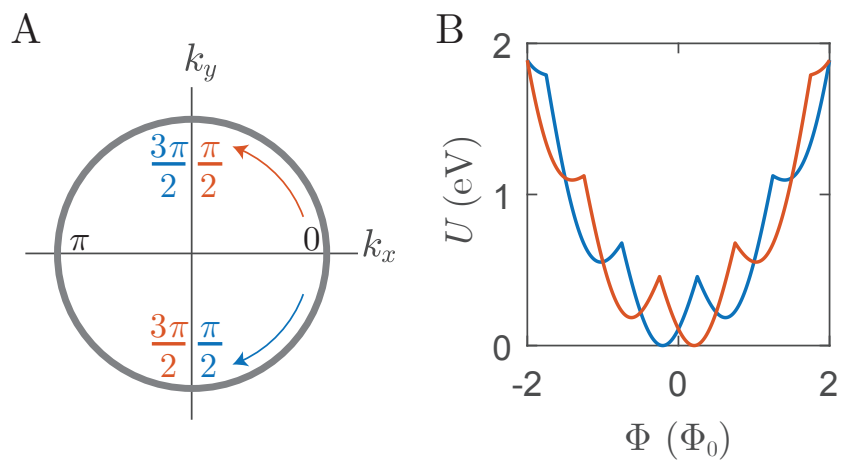

Figure 7.5: A) Magnitude and phase of the order parameter for $p_{x}+i p_{y}$ and $p_{x}-i p_{y}$ symmetry. B) Free energy $U$ as function of the total flux $\Phi$ for $\theta=90^{\circ}$.

energy), such structures would randomly have their angular momentum (and with that, their $\frac{1}{4} \Phi_{0}$ flux) point up or down, all else being equal. While in the $d$-wave case the $\frac{1}{2} \Phi_{0}$ is a degenerate ground state, the $\frac{1}{4} \Phi_{0}$ is not degenerate in the same way, since moving from (for example) $+\frac{1}{4} \Phi_{0}$ to $-\frac{1}{4} \Phi_{0}$ would require a change of chirality. The next available state with the same chirality would be $-\frac{3}{4} \Phi_{0}$, though this is a higher energy state.

We can write down the free energy of the system the same way we did in Chapter 3 (without the correction discussed in Section 3.7), which gives us:

$$
U=\frac{\left(\Phi-\Phi_{e}\right)^{2}}{2 L}-E_{J} \sqrt{2+2 \cos 2 \pi \frac{\Phi}{\Phi_{0}} \pm \theta}
$$

where the \pm sign again depends on the chirality of the system. The free energy is shown in Figure $7.5 \mathrm{~B}$ for both chiralities with $\theta=90^{\circ}$, showing the minima at $\left(n \pm \frac{1}{4}\right) \Phi_{0}$.

Since the phase now increases linearly with $\theta$, we could in theory generate any spontaneous flux between 0 and $\frac{1}{2} \Phi_{0}$ by changing $\theta$ from 0 to $180^{\circ}$. Creating an array of such devices as Kirtley et al. did in Reference [50] should show a nicely increasing flux with increasing $\theta$ following

$$
\Phi=\left(n-\frac{\theta}{2 \pi}\right) \Phi_{0}
$$


A

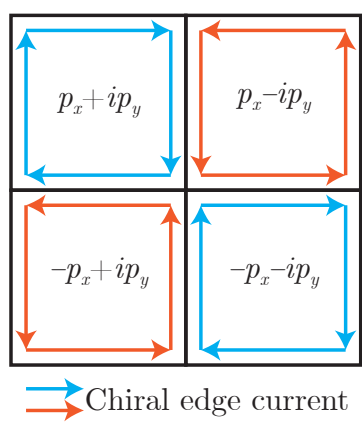

$\mathrm{B}$

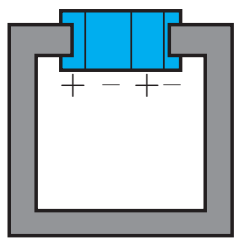

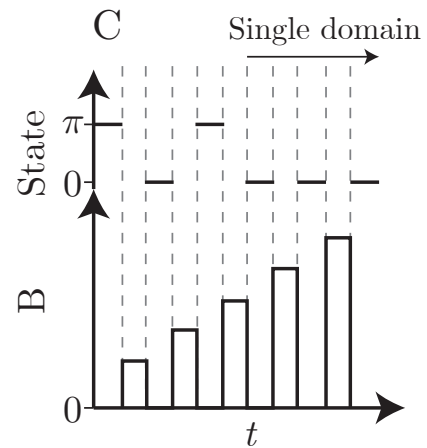

Figure 7.6: A) Chiral domains with edge current and changes in the wave function. B) SQUID with a chiral component with several domains. C) Schematic concept measurement of a SQUID containing a chiral domain structure as in (B). As a function of time $t$, the external field $B$ is increased to a higher value each cycle, then reduced to zero. Afterwards, the state of the SQUID is measured, which will be either 0 or $\pi$.

\subsubsection{Chiral domains and spontaneous edge currents}

Due to the degeneracy of the $p_{x} \pm i p_{y}$ states, larger $p$-wave superconductors are predicted to divide into domains with different chiralities. When crossing the domain wall, the component perpendicular to the domain wall normal vector will phase-shift by $\pi$, while the parallel component stays the same (Figure 7.6A). Asano et al. show that this has implications for a SQUID geometry as shown in Figure 7.4, since both the angle that the supercurrent rotates through as well as the number of domain walls crossed are important [163].

Figure 7.6B shows a layout similar to Figure 7.4A, but with an arbitrary chiral domain structure. As mentioned, for each perpendicular domain wall crossed by the supercurrent, an additional $\pi$ phase shift is obtained. This means that in such a SQUID, the maximum supercurrent $I_{\text {SQUID }}$ will be described by

$$
I_{\mathrm{SQUID}} \propto\left|\cos \left(\pi \frac{\Phi}{\Phi_{0}}+n \frac{\pi}{2}\right)\right|,
$$

where $n$ is the number of domain walls encountered. This means the behaviour of such a SQUID will switch between regular and that of a $\pi$-loop depending on its domain structure. Therefore, the existence of a sponta- 
neous magnetic flux will not only depend on the geometry of the device but also on the internal domain structure of the chiral superconductor.

The domain structure can be influenced by a magnetic field. For example, by cooling through $T_{c}$ in an applied field, or by applying a magnetic field while the material is superconducting, one chirality will be preferred over the other. So one can imagine an experiment where the magnetic field is increased to higher values each time, moved back to zero and then the state of the SQUID (either 0 or $\pi$ ) is determined, either through measuring $I_{c}$ or determining whether or not a spontaneous flux is present. This should oscillate with the magnetic field until the material contains only a single domain, after which the state will be constant (Figure 7.6C). Note again that only the number of domain walls perpendicular to the current direction is relevant.

As shown in Figure 7.6A, the edge currents of two neighbouring domains run in the same direction. This means that we might expect a local magnetic field originating from these currents. For $\mathrm{Sr}_{2} \mathrm{RuO}_{4}$, these magnetic fields are estimated to be on the order of $1 \mathrm{mT}[161,164]$. However, they have so far not been observed with scanning magnetic imaging techniques $[160,161,165]$.

\subsubsection{Vortices}

In type II superconductors, vortices appear in fields above $H_{\mathrm{c} 1}$ carrying a single flux quantum. In chiral superconductors, however, theoretical studies have shown that in some cases, vortices with a flux of $2 \Phi_{0}$ are more stable [166-168]. Additionally, while the classic Abrikosov lattice is a triangular one, both theoretical and experimental findings suggest that at higher fields, vortices in a chiral superconductor arrange in a square lattice instead [168-171].

As discussed previously, SSM is an ideal setup for measuring vortices. It allows for both imaging the distribution of vortices (to see whether they arrange in a triangular or square lattice) as well as accurately measuring the flux carried by an individual vortex. While vortices have been measured in $\mathrm{Sr}_{2} \mathrm{RuO}_{4}$, so far only regular single-quantum vortices were observed $[160,161,165]$. Whether this is because of the material or the theory is unclear. Studying more $p$-wave candidates for these effects can yield more insight into where the problem might be.

In 1974, Kramer and Pesch predicted that at temperatures $T \ll T_{c}$, 
the pair potential in a vortex increases sharply over a length $\xi_{1} \sim \xi T / T_{c}$ [172]. This is interpreted as a 'shrinking' of the vortex core, since the magnetic field decreases more rapidly away from the vortex centre. Since flux quantisation still holds, the vortex will carry an integer multiple of $1 \Phi_{0}$, meaning the magnetic field distribution will have to have higher peak values and decrease on a smaller length scale with decreasing temperature. This so-called Kramer-Pesch effect is predicted to be more apparent in a chiral superconductor because of an inherent suppression of the scattering rate due to non-magnetic impurities [173], though this suppression may only hold for certain kinds of impurity scattering [174].

Of course, measuring such an effect will require very high spatial resolution: the change in $\xi_{1}$ is on the order of $10 \mathrm{~nm}$, meaning changes in the magnetic field will also happen on such scales. SSM sensors with $50 \mathrm{~nm}$ and smaller pickup loops have been created [158], which may not yet be sufficient to observe these effects.

\subsection{Magnetic monopole image charge}

While the magnetic monopole as an elementary particle remains elusive, several systems can have emergent effects that behave like a monopole. In condensed matter physics, the spin-ice class of materials is the best-known example of this. Due to aligning of spins in a string, the two ends will show a monopole-like magnetic field [175].

Here we will look at how a 'monopole' can be induced at the surface of a topological insulator. We start by looking at the free energy $F$ of a material expanded in terms of the electric field $E$ and the auxiliary magnetic field $H$ :

$$
\begin{aligned}
F= & -\sum_{i} P_{i} E_{i}-\sum_{i} M_{i} H_{i}-\sum_{i, j} \frac{1}{2} \epsilon_{0} \epsilon_{i j} E_{i} E_{j} \\
& -\sum_{i, j} \frac{1}{2} \mu_{0} \chi_{i j} H_{i} H_{j}-\sum_{i, j} \alpha_{i j} E_{i} H_{j}+\ldots,
\end{aligned}
$$

where $P$ is the electric polarisation, $\epsilon_{0}$ the electric permittivity of the vacuum, $\epsilon_{i j}$ the relative permittivity tensor, $\chi_{i j}$ the magnetic susceptibility tensor and $\alpha_{i j}$ is known as the linear magnetoelectric susceptibility tensor $[176]$. 


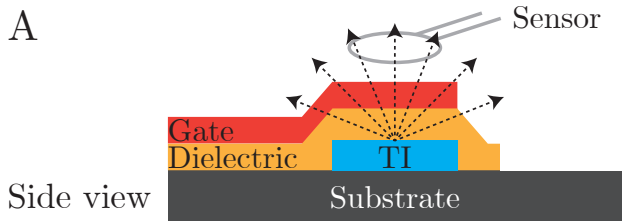

Top view

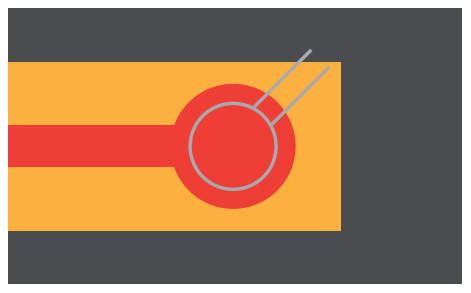

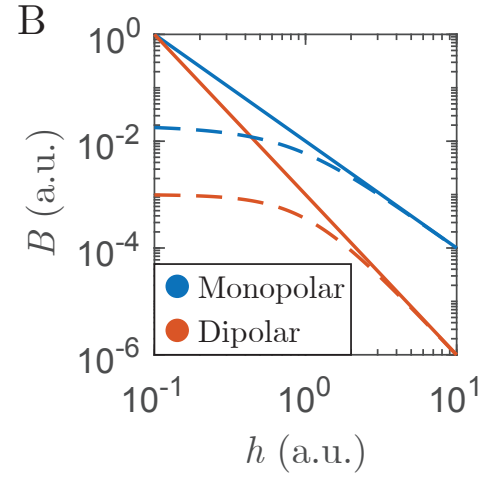

Figure 7.7: A) Schematic device layout to measure the monopole image charge induced by a top-gate using SSM. B) Strength of the magnetic field as function of $h$. Solid lines indicate the actual field strength, dashed lines indicate the influence of a pickup loop with non-zero diameter.

In most materials, $\alpha=0$, and magnetic and electric effects are uncorrelated (e.g., $P$ does not depend on $H$ ). However, in a system where time-reversal symmetry is broken (such as at the surface of a topological insulator), $\alpha$ can be non-zero [176]. Several recent papers have shown that for such a material, placing an electric charge near the surface can result in a monopole-like field outside the material [177-180].

Assuming the material is isotropic, the magnetic field of a monopole with a charge $m_{0}$ is given by [180]:

$$
\begin{gathered}
\vec{B}=\frac{m_{0}}{r^{3}} \vec{r} \\
m_{0}=-\frac{\mu_{0}}{4 \pi} \frac{2 q \alpha}{\left(\mu+\mu_{0}\right)\left(\epsilon+\epsilon_{0}\right)-\alpha^{2}} .
\end{gathered}
$$

In an experiment, we would want to verify two facts. First, that the field produced is actually monopolar. Second, the value of $m_{0}$ (and therefore the strength of the resulting field) should depend on the total charge $q$ present on the sample surface.

Figure 7.7A shows a schematic experimental layout. By fabricating a top-gate on a topological insulator, charge can be accumulated on the surface by applying a gate voltage $V_{g}$ that will induce the magnetic monopole. One important consideration is that the charge will have an effect on the 
topological insulator and possibly move its Fermi level out of the gap, suppressing its topological properties. The SSM can then image an area around the gate to measure the field. The resulting image should appear as shown in Chapter 2, Figure 2.5.

The data can be fit to the model and see if it follows the $1 / r^{2}$ behaviour of a monopole, compared to the $1 / r^{3}$ behaviour of a dipole. This can also be done by measuring $B(h)$ by slowly increasing the distance between sample and sensor and measuring the magnetic field. This should then follow a $1 / h^{2}$ trend as well. This is shown in Figure 7.7B, compared to the field dependence of a point dipole along the $z$-axis. Since the SSM sensor will have a finite diameter $d=2 R$, this will influence the signal that is actually measured, especially in the range $d \gg h$. This is shown by the dashed lines in Figure 7.7B. These functions $\bar{B}$ can be obtained by integrating over the loop area, and are given by

$$
\begin{aligned}
\bar{B}_{\text {monopole }} & =\frac{2 m_{0}}{R^{2}}\left[1-\frac{h}{\sqrt{R^{2}+h^{2}}}\right], \\
\bar{B}_{\text {dipole }} & =\frac{2 m_{1}}{\left(R^{2}+h^{2}\right)^{\frac{3}{2}}},
\end{aligned}
$$

where $m_{1}$ is some dipole moment.

The dependence of $m_{0}$ on the total charge should be easily verifiable by keeping the SSM sensor stationary over the gate, and measuring $B$ while changing $V_{g}$. In principle, the field should change linearly with $q$ (and therefore, $V_{g}$ ), though other effects (like the aforementioned gating of the TI itself) might influence this. In addition, Spanton et al. noted that using an electric gate in proximity to a SQUID sensor can influence its properties and therefore the measurement data [181]. It would thus be wise to verify the influence of the top-gate on the sensor in a dummy experiment.

\subsection{Concluding remarks}

In this chapter we have discussed several interesting properties of topological insulators and superconductors and how they might be studied using SSM. Some of these experiments may require more advanced SSM technology than currently available (for example, the Kramer-Pesch effect discussed in Section 7.4.4). Others, like the observation of 1D edge 
channels, should be possible with current SSM and sample fabrication techniques. Therefore, we showed that the SSM should be a powerful tool in this field.

In addition, there are some other effects which have not been discussed here where SSM may prove useful. For example, in Josephson junctions where the barrier is topologically non-trivial, Majorana bound states cause a doubling of the Josephson periodicity [182]. Such a $4 \pi$-periodic component can possibly manifest itself in the flux through a SQUID and then be imaged using SSM $[183,184]$.

A more exotic proposal involves using spin-momentum locking as a means to store energy [185]. When a current is applied to a TI, the charge carriers will be spin-polarised according to spin-momentum locking. Upon scattering, they will transfer this spin to the nuclei involved in the scattering event. Over time, this will cause nuclear spins to obtain the same spin polarisation as the charge carriers. This process is commonly known as dynamic nuclear polarisation. When the current is removed, the now-polarised nuclear spins will impart their spin to scattering charge carriers, forcing these to move in a certain direction due to spin-momentum locking. In an open circuit, these charge carriers will then accumulate at the boundary, creating a capacitance.

While this concept can be verified using regular transport measurements, the presence of net spins at the boundaries might be visible to an SSM, depending on how much spin can be accumulated. Some early testing of the concept showed no sign of an increased capacitance within the limits of the measurement setup used, though the setup and sample fabrication were not yet fully optimised [186]. 


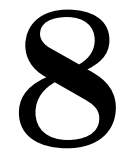

\section{Closing thoughts}

In this chapter we will briefly look back at the various studies performed throughout this thesis and provide some final thoughts. We will also cast a look into the future based on the most recent developments in SSM and related technologies. 


\subsection{Concluding remarks on this thesis}

Throughout this work we have studied a wide variety of material systems using scanning SQUID microscopy supplemented with several other measurement techniques as well as theoretical modelling. Though the studies presented here differ greatly from one another, all of them relate to fundamental questions. These include such diverse topics as the electronic structure of thin film oxides (Chapters 5 and 6) or unravelling the mechanics behind the magnetic field of our planet (Chapter 4).

In this thesis, we have tried to go beyond just imaging the local magnetic field at a sample surface. By fitting the SSM's high resolution field data to models, we have extracted more detailed information about the various systems we studied. Sometimes this was relatively straightforward, such as the dipole-based systems of Chapter 4. Extracting the magnetic moments from the data by fitting it to the dipole equation can give us insights into how these materials work on more fundamental levels.

At other times the SSM allowed us to go even further, such as when we analysed the free energy of the $\pi$-loops in Chapter 3 . The magnetometry data there allowed us to extract the $\beta_{L}$ parameter of these devices, not only giving us the free energy, but also allowing us to determine that current theoretical models used for calculating this free energy may be incomplete. This was possible due to the ability to place the sensor at a specified location and vary the external field. Such an experiment may also be done by other magnetically sensitive techniques, since we only need to know the relative positions of the transition points on the hysteresis curves to extract $\beta_{L}$ (refer to Figure 3.7E). However, the ability of the SSM to confirm the flux states at various points on the hysteresis curve by measuring the total flux is not easily reproduced by other techniques.

In Chapter 2, we made use of modelling and data fitting to learn more about the SSM setup itself. We used data from an imaged Abrikosov vortex and a current line to determine the angle between the sensor and sample surface, and modelled the magnetic shield to determine its effectiveness. We also spent some time looking at simulated ferromagnetic surfaces and how various parameters influence the resulting image.

One aspect we did not cover in Chapter 2 is deconvolution. We touched on it briefly when looking at the influence of the pickup loop diameter in Section 2.4. However, there may be much to gain by developing proper deconvolution, as mentioned in Section 2.5. This is particularly impor- 
tant for studies that are heavily dependent on fitting accuracy, such as those performed in Chapters 3 and 4: since convolution with the sensor shape alters the data, and therefore the values of the fitting parameters, accurate deconvolution could substantially improve measurements. Good deconvolution algorithms are also crucial for studies where accuracy is not necessarily as important, but image quality is: for example, when examining ferromagnetic domain structures like in Chapters 5 and 6.

Although some progress can be made by modelling the sensor as a ring or square object, the real shape is far more complex (see, for example, the study in the supplementary information of Reference [27]). Much can be learned from astronomy, where deconvolution techniques are commonplace to improve the quality of telescope imagery (see Reference [187] for an overview). While there are of course several differences between astronomy and SSM (for example, scanning height is a much more important factor in SSM than the telescope-celestial body distance is in astronomy), it is likely that many of the underlying mathematical theories can be adapted for use with SSM.

Finally, the use of modelling allowed us to make several predictions in Chapter 7 about the feasibility of various experiments on topological insulators. While the estimates were fairly basic, they give us a general idea of how to design an experiment or which sample parameters are more important than others.

As the saying goes, often the sum is greater than the parts. So, too, is the SSM increasingly useful when used as a part of a larger study involving multiple characterisation techniques. The simplest demonstration of this came in Chapter 4, Section 4.4, where we used X-ray tomography in addition to SSM in order to get both positional and magnetic data on the magnetite grains. This allowed us to perform the inversions required to obtain the magnetic moments for each grain.

Chapter 5 went even further, combining SSM measurements with a large set of different techniques in order to study the behaviour of ferromagnetic LMO thin films. The large change in magnetic signal across the critical thickness is neither observed nor satisfactorily explained by the other techniques, implying that the mechanics of magnetism in LMO are more complex than can be established with the other methods alone. Here, the SSM was crucial in highlighting this aspect of the story.

The limited spatial resolution of the SSM, discussed in Section 8.2, can also be improved by combining it with a second magnetically sensitive 
scanning probe technique (e.g., magnetic force microscopy). The SSM can then provide the magnetic field data of an object with extreme accuracy, while the second technique can look at the magnetic structure and see which regions are magnetically active. Combining the two datasets can then yield a more complete model of the object.

\subsection{On the limits and future of scanning SQUID microscopy technology}

The high, direct sensitivity of the SQUID to magnetic field strength is what makes SSM such a powerful technique. This separates it from such techniques as magnetic force microscopy, which is sensitive to a force rather than the field, or scanning Hall bar microscopy, which has far lower sensitivity.

However, the use of a SQUID also puts a significant limit on the spatial resolution of the system. While not a very complicated structure per se, attempting to fabricate a SQUID (or pickup loop) of increasingly smaller scale is largely a technological challenge. Moving to electron-beam lithography or focused ion-beam milling methods is an option, allowing the structuring of features with dimensions down to roughly $10 \mathrm{~nm}$. A completely different method was developed by the Zeldov group at the Weizmann Institute in Israel, who managed to deposit a SQUID on the tip of a quartz tube pulled to a diameter less than $100 \mathrm{~nm}$ [157].

However, even though the physical dimensions of the SQUID can be reduced, its other parameters can suffer as a result. For example, with smaller leads and Josephson junctions, the critical current $I_{c}$ will become smaller as well. In order to maintain the Josephson coupling, the Josephson energy $E_{J}$ should be much larger than the thermal energy $k_{B} T$. At $4 \mathrm{~K}$, this would translate into having a critical current $I_{c}>0.18 \mu \mathrm{A}$. Naturally, this value becomes even smaller when going down to sub-kelvin temperatures, such as when performing experiments in dilution fridges and similar settings.

Similarly, reducing the sensitive area of the SQUID pickup loop will directly impact its field resolution. Since $\Phi=B \cdot A$, a significant reduction in area means the magnetic field needs to change that much more in order to get a similar response from the system.

To some extent, the reduction in field sensitivity may be offset by the 
ability to reduce the distance between the sample and the sensor. For example, the field from a dipole falls off as $1 / r^{3}$, meaning that reducing the separation from $1 \mu \mathrm{m}$ to $100 \mathrm{~nm}$ can yield a gain in signal strength of up to 3 orders of magnitude. This is one large advantage of the SQUIDon-tip design, which can approach a sample surface much more closely than a regular chip-based sensor (50 nm versus $330 \mathrm{~nm})[7,87,157]$.

An interesting geometry used by some groups involves incorporating a flux guide [188-191]. Instead of bringing the SQUID sensor as close to the sample surface as possible, the SQUID is placed at the wide end of a needle, typically made out of permalloy or $\mu$-metal. The tip of the needle is then brought as close as possible to the sample surface, picking up the magnetic field and funnelling it to the SQUID. While these flux guides have mostly been used to reduce the sensor-sample distance in setups where the sample is outside the sensor cryostat (see below), one might imagine using such a flux guide to achieve higher spatial resolution. A hybrid scanning tunnelling microscope - SSM with a spatial resolution of $1 \mu \mathrm{m}$ was built by Tachiki et al. based on this idea [192].

Temperature-wise, an SSM is obviously bounded by the critical temperature of the SQUID on the high end. Some systems have been designed where the sample is outside the SSM cryostat, with a thin window separating the two [193-195]. However, this severely limits the spatial resolution since the sample-sensor distance is significantly increased.

Of course, another option is to move to SQUIDs made out of high- $T_{c}$ materials. A few groups have built SSM systems with sensors using YBCO as a high- $T_{c}$ superconductor $[191,196,197]$. However, most of the studies using SSM are on materials with interesting effects at temperatures near absolute zero. This, combined with the relative ease of structuring $\mathrm{Nb}$ compared to YBCO, means not much effort has been put into high- $T_{c}$ sensors in recent years.

The Moler group at Stanford University in the US, as well as the Kalisky group at Bar-Ilan University in Israel, have developed an SSM system in a cryogen-free setup, with the sensor thermally isolated from the sample but within the same space $[198,199]$. This way, the Moler group achieved a sample temperature range between 2.8 and $110 \mathrm{~K}$ [198].

For lower temperatures, it is not necessarily the SQUID itself that becomes the problem but the scanning stage. The choice for scanning stages usually falls between mechanical and piezoelectric actuators. Considering the limited amount of space inside sub-kelvin coolers, piezoelectric 
actuators seem to be the logical choice. However, the displacement range of piezoelectrics shrinks significantly in cryogenic environments, limiting the sample area that can be imaged. Fortunately, several suppliers now provide piezoelectric scanning systems optimised for sub-kelvin use.

Recently, the Zeldov group has used their SQUID-on-tip system as a nanoscale thermal sensor [158]. The local sample temperature is determined by measuring the changes in the properties of the SQUID, leading to a thermal sensitivity of $870 \mathrm{nK} / \sqrt{\mathrm{Hz}}$. Such a concept only works because the SQUID can be brought in such close proximity to the area of interest; a sensor extended with pickup loop that is typically used in SSM systems would not be able to achieve such thermal sensitivity.

This demonstration of thermal sensitivity shows that the capabilities of a scanning SQUID system can be extended beyond imaging magnetic fields. It might be feasible to image any physical property that can influence the properties of the SQUID, such as a local accumulation of charge or emission of photons.

Throughout this thesis we have supplemented SSM data with data from other measurement techniques, as well as computer modelling and fitting. This was especially useful in Chapter 4, Section 4.4, where we used X-ray tomography to obtain the positional data required to uniquely invert the SSM data. Proper inversion remains difficult to do in a timely or accurate manner, due to the high number of non-linear variables, so reducing this number is crucial. X-ray tomography is but one way to do this; other possibilities include atomic force microscopy or scanning electron microscopy for surface features such as devices or adsorbates.

However, these techniques are only useful for well-defined and wellseparated magnetic features, such as those studied in Chapter 4. For ferromagnetic films, like LMO on STO in Chapters 5 and 6 , this is a more daunting task. A ferromagnetic domain structure is much harder to image: this is typically done with magnetic force microscopy. An additional problem is that the domain structure, depending on the material, is not always stable, especially when samples need to be heated and cooled multiple times to transfer from one measurement setup to the next.

The basic SSM setup is only sensitive to the $z$-component of the magnetic field. Of course, the magnetic field is a three-dimensional vector quantity, meaning that these systems are only sensitive to one-third of the available information. To that end, the Ishida group at Osaka University in Japan have recently developed a vector scanning SQUID sys- 
tem [200, 201], after an early study in 1997 by Ketchen, Kirtley and Bhushan [202]. Tripling the amount of available data should make inversion procedures yield more accurate results.

All in all, while scanning SQUID microscopy is a relatively niche technique, advancements are still being made on a regular basis. These advancements take the form of not only spatial and field resolution or increased temperature ranges, but also analytical methods and computing power to perform inversions with larger datasets. This ensures that SSM can keep its place as a strong characterisation method in the field of materials science. 


\section{Samenvatting}

In dit werk hebben we de magnetische eigenschappen van verschillende vastestofsystemen bestudeerd. Hiermee hebben we gepoogd meer te weten te komen over het materiaal zelf of de omstandigheden waarin het gevormd is. Dit hebben we gedaan door de data te vergelijken met theoretische voorspellingen en te fitten aan modellen om relevante parameters te bepalen.

In Hoofdstuk 2 hebben we vooral gekeken naar de opstelling zelf en hoe de scanning SQUID microscoop (SSM) bepaalde magnetische fenomenen afbeeldt. Door specifieke structuren te scannen hebben we meer inzicht gekregen in sommige aspecten van de opstelling, zoals de hoek tussen de sensor en het monsteroppervlak, en de hoogte van de sensor. Zo hebben we kunnen bepalen dat deze hoek significant hoger is dan ontworpen $\left(40^{\circ}\right.$ tegenover $\left.27^{\circ}\right)$, wat betekent dat de sensor gevoeliger is voor de magnetische-veldcomponent in het vlak. We hebben het afbeelden van Abrikosov vortices gemodelleerd en gekeken hoe de gemeten totale flux afhangt van de integratieoppervlakte. Aangezien het bestuderen van Abrikosov en andere soorten vortices een veelvoorkomend doel is van de SSM, is zulke informatie belangrijk om te bepalen hoe accuraat dergelijke metingen zijn. Als laatste hebben we het afbeelden van ferromagnetische oppervlakken onderzocht. Door de gemiddelde veldsterkte uit te drukken in een getal $B_{\mathrm{RMS}}$, konden we bepalen hoe dit getal afhangt van verschillende parameters zoals de hoogte van de sensor of domeingrootte.

In Hoofdstuk 3 hebben we supergeleidende structuren bestudeerd, die bestaan uit $s$-wave en $d$-wave supergeleiders. In specifieke geometrieën kunnen zulke structuren spontane magnetische flux laten zien zonder dat er externe velden of stromen worden aangelegd. Daarbovenop komt het

feit dat deze spontane flux wordt beschreven als $\left(n+\frac{1}{2}\right) \Phi_{0}$, in tegen- 
stelling tot the reguliere fluxkwantisatie $n \Phi_{0}$. Door het gebruik van een geïntegreerde veldspoel op de chip hebben we laten zien dat we kunnen schakelen tussen de twee grondtoestanden $-\frac{1}{2} \Phi_{0}$ en $+\frac{1}{2} \Phi_{0}$. De keuze voor een andere supergeleider (MoRe) met een hogere kritische stroom stelde ons in staat hogere fluxtoestanden te stabiliseren. Ook hebben we lokale magnetometriemetingen gedaan waarmee we het $I_{c} L$-product van de structuren konden bepalen en de vrije energie konden berekenen. We lieten zien dat de huidige beschrijving van de vrije energie mogelijk incompleet is, aangezien er een verschil is tussen de hoeveelheid stabiele toestanden in het model en in het experiment.

Daarna hebben we gekeken naar een aantal verschillende systemen die zich gedragen als (een verzameling van) dipolen in Hoofdstuk 4. Eerst hebben we kort monsterverontreinigingen bestudeerd en hoe deze te zien zijn in SSM-afbeeldingen. We zagen dat verontreinigingen er meestal uitzien als dipolen gericht in het vlak. Afhankelijk van het systeem dat bestudeerd wordt, kan men mogelijk deze verontreinigingen vinden en uitsluiten tijdens de analyse om de kwaliteit van de data te verbeteren. Daarna volgde een studie op $\mathrm{Fe}_{3} \mathrm{Se}_{4}$ magnetische nanodeeltjes. Door de SSM-data van deze deeltjes te fitten aan de dipoolvergelijking, konden we de grootte en richting van het magnetische moment bepalen. Dankzij de schijfvorm van de deeltjes zijn de meeste magnetische momenten gericht in het vlak. We zagen echter geen voorkeursrichting in het vlak zelf, zelfs nadat de deeltjes langs een specifieke richting gemagnetiseerd waren, wat op een hoge magnetische anisotropie duidt. De grootte van een typisch magnetisch moment was groter dan we verwachtten op basis van eerdere experimenten en theoretische voorspellingen, wat mogelijk komt door fouten in de schatting van de grootte van deze deeltjes. Als laatste hebben we magnetische deeltjes in geofysische monsters onderzocht. Door deze deeltjes te bestuderen kunnen we meer leren over het magnetisch veld van de aarde en hoe dit verandert op geologische tijdschalen. We hebben een artificieel monster gemaakt om een nieuwe meetmethode, die SSM met computertomografie combineert, te kunnen testen. Deze meetmethode levert zowel magnetische als positionele data op. Door deze data te combineren wordt de inverseberekening, waarmee het magnetisch moment bepaalt wordt, significant eenvoudiger. We hebben laten zien dat deze methode een goede vervanging zou kunnen zijn voor huidige meetmethodes in dit onderzoeksveld, die veelal inaccuraat en destructief zijn.

In Hoofdstukken 5 en 6 hebben we aandacht besteedt dunne films van 
de complexe oxidenLaMnO $\mathrm{LaAlO}_{3}$ en $\mathrm{LaArTO}_{3}$ substraten. In Chapter 5 hebben we het magnetische karakter van $\mathrm{LaMnO}_{3}$ bestudeerd met behulp van verschillende karakterisatiemethoden. We zagen onder meer dat de meeste Mn-ionen een Mn3+-karakter hebben, met wat $\mathrm{Mn}^{2+}$ in de buurt van het raakvlak met het $\mathrm{SrTiO}_{3}$. We observeerden ook ferromagnetische signalen in alle geteste monsters, die variëerden in dikte van 2 tot 10 uc. Deze bevindingen spreken het zogenoemde 'polar catastrophe'model tegen, dat gesuggereerd werd om de observatie van een kritische dikte voor ferromagnetisme rond 6 uc te verklaren.

Daarna bestudeerden we heterostrukturen van $\mathrm{LaMnO}_{3}$ en $\mathrm{LaAlO}_{3}$ in verschillende configuraties en met verschillende diktes in Hoofdstuk Chapter 6. We zagen dat het tweedimensionale electronengas aan het $\mathrm{LaAlO}_{3}-\mathrm{SrTiO}_{3}$ raakvlak een verhoogde mobiliteit en een lagere ladingsdragersdichtheid had wanneer we een $\mathrm{LaMnO}_{3}$-toplaag toevoegden. Dit hebben we verklaard door te stellen dat het $\mathrm{LaMnO}_{3}$ als een katalysator werkt om zuurstofvacatures op te vullen. De heterostructuren lieten ook twee magnetische fases zien, waarvan één aan het $\mathrm{LaMnO}_{3}$ toebehoort en de andere aan het $\mathrm{SrTiO}_{3}$ substraat. In het omgekeerde geval zagen we dat $\mathrm{LaMnO}_{3}$-films met een $\mathrm{LaAlO}_{3}$-toplaag geen geleiding lieten zien, ongeacht de filmdikte. Daarentegen nam het magnetische moment van het $\mathrm{LaMnO}_{3}$ significant toe als een $\mathrm{LaAlO}_{3}$-laag werd toegevoegd. Dit is mogelijk gerelateerd aan een verandering in de zuurstofverhouding in het $\mathrm{LaMnO}_{3}$ of aan het completeren van de zuurstofoctahedra aan het $\mathrm{LaMnO}_{3}$-oppervlak.

Ten slotte hebben we in Hoofdstuk 7 enkele interessante eigenschappen van topologische isolatoren beschreven en hoe we deze met de SSM kunnen observeren. In tweedimensionale toplogische materialen lopen stromen langs de randen van het materiaal en het resulterende magnetisch veld kan met de SSM afgebeeld worden. Daarnaast zijn spin en impuls sterk gekoppeld in deze materialen, wat betekent dat een stroom een netto spin zal hebben. Hoewel dit een magnetisch veld creëert, zal dit veld, uitgaande van typische waardes voor deze materialen, te klein zijn ten opzichte van het veld veroorzaakt door de stroom zelf om het hiervan te kunnen onderscheiden. Ook hebben we kort gekeken naar $p$-wave supergeleiders en hoe de SSM gebruikt kan worden om een onderscheid te maken tussen deze en andere symmetrieën. Als basis hebben we dezelfde structuren bekeken als die in Hoofdstuk 3, welke een spontane magnetische flux zouden moeten laten zien in specifieke configuraties. Als laatste 
Samenvatting

hebben we gekeken naar het afbeelden van een magnetische spiegellading die zou kunnen verschijnen wanneer een elektrische lading boven een topologische isolator geplaatst wordt. Enkele theoretische studies suggereren dat een dergelijke magnetische spiegellading een veld produceert dat eruitziet als het veld van een magnetische monopool. We hebben gekeken naar hoe een dergelijke structuur eruit zou moeten zien en hoe de SSM onderscheid kan maken tussen het veld van een monopool en een dipool. 


\section{Summary}

In this thesis we have studied the magnetic properties of various solid state systems. From this we attempted to learn more about the material itself or the conditions in which it was formed. We did this by comparing the data with theoretical predictions and fitting to models to extract parameters of interest.

In Chapter 2 we mostly focused on analysing the setup itself and how the scanning SQUID microscope (SSM) images certain magnetic phenomena. Scanning some specific features allowed us to gain insight in some aspects of the setup, such as the angle between the sensor and sample surface and the sensor height. We found that the scanning angle is significantly higher than designed $\left(40^{\circ}\right.$ versus $\left.27^{\circ}\right)$, which means the sensor will pick up more of the in-plane magnetic field component. We modelled the imaging of Abrikosov vortices and studied how the total measured flux depends on the integration area. Since the study of Abrikosov and other types of vortices is a common application of SSM, such information is important to determine the accuracy of flux measurements. Finally, we studied the imaging of ferromagnetic surfaces. By expressing the overall field strength using a measure $B_{\mathrm{RMS}}$, we were able to determine the dependence of this measure on parameters such as the sensor height or domain size.

In Chapter 3 we studied superconducting devices made out of $s$-wave and $d$-wave superconductors. In specific geometries, such devices can create spontaneous magnetic flux without the application of external fields or currents. Furthermore, the value of the spontaneous flux follows $\left(n+\frac{1}{2}\right) \Phi_{0}$, as opposed to the regular flux quantisation $n \Phi_{0}$. Using an on-chip bias line, we showed that we can switch between the two ground states $-\frac{1}{2} \Phi_{0}$ and $+\frac{1}{2} \Phi_{0}$. By switching to a different superconductor (MoRe) with a 
higher critical current, we were able to stabilise higher flux states. We performed local magnetometry measurements using the SSM to extract the $I_{c} L$ product of the devices and gain insight into the free energy diagram. We showed that the current description of the free energy may be incomplete, due to a mismatch between the observed and predicted number of stable states.

We then focused on several different systems that behaved as (collections of) dipoles in Chapter 4. First we had a short look at sample contaminations and how they can appear on SSM imagery. We observed that contaminants typically appear as in-plane dipoles. Depending on the system under investigation, one might be able to find and exclude these contaminants from analysis to improve the quality of the data. We then moved to $\mathrm{Fe}_{3} \mathrm{Se}_{4}$ magnetic nanoparticles. We fitted the SSM data from these particles to the dipole equation, which allowed us to extract the magnetic moment vector. The vectors lie mostly in-plane, owing to the disc-shape of the particles. However, no preferred in-plane orientation was observed, even after magnetising the particles along a specific direction, indicating high anisotropy. The magnitude of the typical magnetic moment we found was higher than expected based on earlier experiments and theory, possibly due to errors in the size estimate of the particles. Finally we looked at magnetic grains in geophysical samples. By studying these particles, we can learn more about the earth magnetic field and how it evolves on geological timescales. We fabricated a custom sample as a proof of concept and combined SSM data with X-ray tomography to gather both positional and magnetic field data. This combination of data significantly simplifies the inversion required to obtain the magnetic moment vector. We showed that this method could be a solid replacement for current methods used in the field, which are typically inaccurate and destructive in nature.

Chapters 5 and 6 were dedicated to the study of thin film complex oxides $\mathrm{LaMnO}_{3}$ and $\mathrm{LaAlO}_{3}$ on $\mathrm{SrTiO}_{3}$ substrates. In Chapter 5 we used various characterisation techniques to study the magnetic character of $\mathrm{LaMnO}_{3}$ thin films. We found that most $\mathrm{Mn}$ ions are of $\mathrm{Mn}^{3+}$ character, with some $\mathrm{Mn}^{2+}$ near the interface with $\mathrm{SrTiO}_{3}$. We also measured ferromagnetic signatures across all tested film thicknesses (between 2 and $10 \mathrm{uc}$ ). These findings contradict the proposed 'polar catastrophe' model used to explain an observed magnetic transition around $6 \mathrm{uc}$.

Then in Chapter 6 we studied heterostructures of $\mathrm{LaMnO}_{3}$ and $\mathrm{LaAlO}_{3}$ 
in different configurations and with varying thickness. We found that the two-dimensional electron gas at the $\mathrm{LaAlO}_{3}-\mathrm{SrTiO}_{3}$ interface has increased mobility and a lower carrier density upon adding a $\mathrm{LaMnO}_{3}$ capping layer. This is attributed to the filling of oxygen vacancies, possibly catalysed by the $\mathrm{LaMnO}_{3}$ layer. The heterostructures also showed two magnetic phases, one from the $\mathrm{LaMnO}_{3}$ layer and one from the $\mathrm{SrTiO}_{3}$ substrate. In the reversed case, we found that the $\mathrm{LaAlO}_{3}$-capped $\mathrm{LaMnO}_{3}$ thin films showed no conductivity at any thickness. However, the magnetic moment in the $\mathrm{LaMnO}_{3}$ is significantly increased with the addition of the $\mathrm{LaAlO}_{3}$. This is possibly related to a change in oxygen stoichiometry in the $\mathrm{LaMnO}_{3}$ or completion of the oxygen octahedra at the $\mathrm{LaMnO}_{3}$ surface.

Finally in Chapter 7, we briefly discussed some interesting properties of topological insulators and how we can use the SSM to observe these. In two-dimensional topological materials, current runs along the edges of the material and the resulting field can be imaged by SSM. In addition, spin and momentum are coupled strongly in these systems, meaning a current will have a net spin. While this results in a magnetic field, it will be too small (using typical material parameters) compared to the magnetic field originating from the current to be distinguishable. We also looked briefly at $p$-wave superconductivity and how SSM can be used to distinguish it from other order parameter symmetries. As a basis, we used the same type of devices used in the experiments in Chapter 3, which should show spontaneous flux generation in specific configurations. Lastly, we looked at imaging magnetic image charges that could arise when electric charges are placed above a topological insulator. Some theoretical studies suggest this magnetic image charge produces a field that looks like that of a magnetic monopole. We looked at what such a device would look like and how the SSM can discern between the field of a monopole and that of a dipole. 


\section{Acknowledgements}

For those that made it this far, well done! For those that skipped this far, well, I have done exactly the same with most theses that came before this, so I will let it slide.

Science is never done alone, and to me, it seems strange to only put my name on the front, even though the contents of this book are the result of collaborations with dozens of people. So here, I would like to thank those that worked with me, supported me or otherwise made some contribution, big or small, over the past 4 years.

I would like to start off with Hans. You have been my supervisor not just for my $\mathrm{PhD}$ period, but also for my Bachelor and Master graduation assignments. So would like to thank you not only for the supervision during the past 4 years, but for almost double that amount. Thanks to you I was able to work on the scanning SQUID microscope for half a decade. While the DESCO programme was focused on the physics of complex oxide thin films, you gave me the freedom to explore many other topics during my $\mathrm{PhD}$, some of which were included in this book. For this I am also very grateful.

Of course I also want to thank Wilfred for being my second supervisor over the past 4 years. While basically all my work was performed in the ICE group, you were nevertheless always prepared to provide feedback and insight. Sometimes the 'third party out-of-the-box' thinking was exactly what was required. I am also very thankful for your dedication and help to our most-beloved Saint Nicholas, which to this day remains my favourite holiday.

These past 4 years I did interesting research and worked on interesting projects. But best of all, I worked with a group of interesting people. Primus inter pares here that I would like to thank is Sander. When 
I came to this university just over 10 years ago, you were chairman of Arago. From afar, you seemed to me to be the perfect student stereotype: lots of beer, lots of parties and activities, not so much actual studying. And while back then those things may have been true (and today, some of those still are!), getting to know you over the past 5 years has shown me that you are an incredibly dedicated person. Not just to science, but to the people around you as well. I would like to thank you (again) for being someone to look up to over the course of our PhDs, for inspiring me to work on some of my personal flaws, for being my captain on several sailing trips, and for sharing many a beer at many a bar many a time.

Second I would like to thank Jorrit. We started on our PhDs around the same time, and I remember one of the first things I did as a $\mathrm{PhD}$ was checking your Master report. We started upstairs in the big office, annoying our fellow students with classical music in the mornings and classic rock music in the afternoons. While we worked on very different topics (though we managed to fix this at the very end), we definitely banded together on many side projects. The office big data file, several iterations of the World/Euro Cup Poule, the ICE Twitter feed, ILSE, the sound board, to name a few! Your western directness was always a nice balance to my more reserved nature. You present yourself as the joker in the group, but I've come to learn that behind the clown mask is a compassionate person that will always help you out if you need it.

Next up is Joris. We actually started at the university at the same time, though we didn't interact much until our time in the group. What has always amazed me about you is that you seem interested in basically everything. Over the past years I've seen you pick up a most varied range of hobbies and pastimes, ranging from stock trading (whether real or in the form of FIFA player cards) to politics to learning Chinese. But unlike most people that half-ass something for a week and then drop it, you always give it your all, which is something I admire about you. And this is just as true for your work in TeaMBE. Much like your big bud Jorrit you were always there if anyone needed help, no matter what. And thanks (I guess?) for showing me that there are indeed people that enjoy Merol!

Martijn is a bit of an enigma. You've mostly preferred to work at home, meaning you were not around often, especially in the later stages of your $\mathrm{PhD}$. Nevertheless, I always very much valued you as a discussion partner, owing to our overlapping interest in SQUID physics and simulations thereof. You've amazed me with how insightful you tried to make all 
these equations, and the insane amount of work you put in your JJAsim package. But we also spent plenty of time discussing all kinds of things, especially during the long trips to and from Delft in our first year, or during our trip to Sardinia. Sardinia was also the place where we hatched the idea for our greatest achievement in this group: the ICE band. So additional thanks for making me pick up the drums!

Speaking of the ICE band, this would have never worked without our lead singer Bob. You are usually not very outspoken, mostly because it seems that you choose your words carefully. You always boil down things to their essence, things don't need to be more complicated than they are! And as mentioned, you took on the role of lead singer for the ICE band, something I did not expect from you, but very glad that you did. You also amaze me by being good at practically every sport we have come across the past years. Only stuff that requires high flexibility seems to get you down, although never for lack of trying!

Then we get to the first of our new generation of PhD students. Daan, you have quickly established yourself as the tech-wizard here in ICE. One of the first things you took on was writing brand new code for almost all our measurement setups, making sure electronics and other equipment could be swapped in and out with zero effort. I would have loved to have worked with you to extend this to the SSM. Your wizardry extends to your mastery of music. You gladly joined the SSM band and blew us away with your skills on the keyboard and bass. Playing through Muse's Hysteria with you leading with that awesome riff was always a highlight of band practice for me. And of course I will never forget our adventure with Bob to see Muse in real life, only to get stranded 10 minutes away from the venue!

I also want to thank honourary ICE member Martina. Things started a little rocky during your Master graduation assignment, until I took over your supervision. Together we figured things out and you wrote it down in a literal prize-winning report, making me extremely proud of what you have accomplished, owing to your unlimited determination to get things done right. Since then, we have spent our time doing far more enjoyable things, and you have become my best metal-concert-buddy. When Elizabeth came over from the US, you two also quickly hit it off and I'm very thankful you helped her feel at home here in the Netherlands. And always remember rule \#1: komt wel goed!

Then I would like to thank my two wonderful, wonderful paranymphs. 
Luca, while I've not known you as long as some of the others, I'm very glad our shared office time fixed that right up. Jorrit often jokes that you are Sander 2.0, but I do have to agree with him. Not because you like taking frequent holidays, have a voice that can be heard from down the hallway or sometimes end up staying at the bar a few more hours than you intended. No, I agree because you, like Sander, are an incredibly hard worker and very dedicated to what you do. You excellently handle multiple collaborations that stretch all over the globe, while managing your dual citizenship in Enschede and Rotterdam. When I proposed having a more involved discussion about student supervision and actually proposing changes, you were right there to pick it up and take it to a higher level, resulting in our recently-started series of $\mathrm{PhD}$ meetings. Thanks for your wonderful support the last few months, for your inspiring people skills and assertiveness, and for being at my side during my defense.

Liesbeth, like with Luca, I've not known you that long. But in that short time, I've gained a truly great 'colleague'. One of your best traits is also your most obvious: your seemingly unending pool of happiness. It is actually hard to be unhappy with you around! But you are much more than that. Behind the smile is someone who always gives everything she's got, something you share with your old supervisor Joris. You somehow manage to not only work full-time at a $\mathrm{PhD}$, but also run your own aerial gymnastics studio at the same time. This has shown me that you have amazing strength, not only physically, but mentally as well, both of which I envy. Speaking of which, I cannot possibly have this paragraph without thanking you for dragging (should I say, wrapping?) me into aerial silks. I'm eternally grateful that you successfully reignited my love for gymnastics and that I get to have such a wonderful teacher. I hope we get to laugh, climb, wrap and drop for years to come!

Alexander and Chuan, while my work unfortunately had little overlap with your projects, you were nevertheless always available for questions or some brainstorming. Thanks to you and your teams I got a nice view into topological materials and I hope someday you will be able to perform some interesting SSM experiments on these intriguing materials.

Of course this rag-tag band of young scientists would be nowhere without our two fantastic technicians. Frank and Dick, I don't think a single paragraph could do justice to the work you two do for the group. You build and maintain our systems, do all our electronics and cryogenics, keep us supplied with substrates and AFM tips, introduce new students to the 
various deposition and fabrication techniques, the list goes on. And then there is the actually important stuff. You two have perfectly assumed the roles of Statler and Waldorf, providing the necessary comic relief between all the scientific discussion. You are also always involved in the various side activities the group has, like the sailing and skiing trips, mountainbiking and ice skating, ice cream making and so many more.

Similarly, this group would surely fall apart without the wonderful administrative skills of Ans. You are always ready to help out, and I've always very much appreciated your no-nonsense approach to your work. All these rules and regulations that just get in the way of getting things done, but you always know exactly what to say and who to call.

There are many, many more group members, current and past, that I would like to thank. First I would like to thank all the students I have supervised over the years. André, Timothy, Martina, Lennard and Sebastiaan, Dave, Bart and Dimas, I have learned much from guiding you through your assignments and hope you learned at least as much from me. And of course these thanks are extended to all other students we have had in the group whom I have helped at one point or another. As PhDs, we sometimes tend to forget that the university is a teaching institute first, research institute second, but nothing feels better than having a group filled with enthusiastic students that are great to work with.

And after all of this, there is a complete second group I need to thank. To all the Nanoelectronics members, I also extend a sincere thank-you. While $99 \%$ of my work was done in the ICE group, it was always a pleasure to spend some time with you. Presenting my work to you in the group seminars forced me to think about how to show my work to people that are not involved, which definitely improved my skills. Of course, the NEvents were always a great pleasure, they made me feel a proper part of the group even though I wasn't present often. I just want to specifically mention Yigitçan, our little oxide enclave in the silicon hive. It was great having you around and talking about actual oxide electronics, instead of the super fundamental stuff we work on at ICE. Our trip down to Switzerland for the IWOE with Sander was also a most enjoyable one. I will be forever sad I had to miss your wedding, and I wish you and Pelin all the best!

The reader will have noticed that many of the projects in this thesis are the result of several collaborations. The unique nature of the SSM setup gave me an opportunity to work with many different groups on many different materials and systems over the last few years. In no particular 
order, I want to thank Lennart and Annemarieke, Renshaw, Hans, Binbin, Kees and Robbert, Pegah, Silvia, the Ariando group, Michael, Nikita, Georgios and Mark, Nicolas, Rossitza and Benjamin, and everyone else I got to have over in 'my' lab. I much enjoyed working, designing, thinking, frustrating, measuring, analysing and writing with all of you.

Then there is a whole bunch of people that did not support me professionally, but definitely made sure I maintained my sanity. The first of these are Steven, Bart and Camiel. We've been a little quartet for over 15 years now, and while we don't see each other as often as we used to, each time it feels like no time has passed at all. I always look forward to the next time we get together to play games, and it's been wonderful seeing each of us go in very different directions but stick together through it all.

The brilliant, just, assertive and brave people of BRAM also deserve many thanks. Together we made our way through the physics programme, and you were a fantastic group of people to be with as a shy little first-year student diving into the great unknown. I hope we will have many more little trips in the future, reminiscing about Kreisintegralen over bananas and how to flirt with girls using tells.

An offshoot of the BRAM collective, my small Magic group also deserves some thanks. Playing together every few months is always great fun, including listening to Chris complaining about work, Harmen talking about his latest 3D-printing mishaps or Wouter discussing his latest Go strategies.

Finally I would like to thank the people closest to me. Pa en Ma, you have been supportive of me for as long as I can remember (literally). No matter the time, date, place or whatever the problem might be, you are always there to help me out if I need it. Everything I have achieved and will achieve is thanks to your immeasurable love and dedication to me and my brothers. Dirk and Sjors, while we are much older (and none the wiser) now, someone looking from afar would think we still behave as five-year-olds sometimes. While we have each gone in very different directions, we always manage to find our way back home and have a good time together.

Lastly, I have to thank my dear Sayya. While it's not always easy, I nevertheless value all the support you have given me over the past few years. The strength you have shown, managing to keep going through everything you're going through, is nothing short of inspiring. While you correcting every last comma in my thesis was definitely helpful, it was 
only a drop in a bucket compared to everything else you've done. I look forward to filling many more years with you with books, films, series, games, dungeons, dragons, concerts, stupid discussions, even more stupid discussions, Oxford commas and whatever else comes on our path. Boop! 


\section{List of publications}

1. P. Reith, X.R. Wang, H. Hilgenkamp, Analysing magnetism using scanning SQUID microscopy, Review of Scientific Instruments vol. 88, 123706, 2017.

2. L.V. de Groot, K. Fabian, A. Béguin, P. Reith, A. Barnhoorn, J.W.M. Hilgenkamp, Determining individual particle magnetizations in assemblages of micro-grains, Geophysical Research Letters, 2018.

3. M.H. Rashid, J. Rabeya, M.H. Doha, O. Islam, P. Reith, G. Hopman, H. Hilgenkamp, Characterization of single step electrodeposited $\mathrm{Cu}_{2} \mathrm{ZnSnS}_{4}$ thin films, Journal of Optics, 2018.

4. M.I. Faley, P. Reith, V.S. Stolyarov, I.A. Golovchanskiy, A.A. Golubov, H. Hilgenkamp and R.E. Dunin-Borkowski, $\pi$-loops with ds Josephon Junctions, IEEE Transactions on Applied Superconductivity, 2018.

5. H. Boschker, T. Harada, T. Asaba, R. Ashoori, A.V. Boris, H. Hilgenkamp, C.R. Hughes, M.E. Holtz, L. Li, D.A. Muller, H. Nair, P. Reith, X. Renshaw Wang, D.G. Schlom, A. Soukiassian, J. Mannhart, Ferromagnetism and conductivity in atomically thin $\mathrm{SrRuO}_{3}$, Physical Review X, 2019. 


\section{Papers in preparation for publication}

1. M.I. Faley, P. Reith, C. Satrya, V.S. Stolyarov, B. Folkers, A.A. Golubov, H. Hilgenkamp, R. Dunin-Borkowski, MoRe/YBCO Josephson junctions and $\pi$-loops, submitted to Superconducting Science and Technology.

2. N. Lebedev, M. Stehno, A. Rana, P. Reith, N. Gauquelin, H. Hilgenkamp, A. Brinkman, and J. Aarts, Gate-tuned anomalous Hall effect driven by Rashba splitting in intermixed $\mathrm{LaAlO}_{3} / \mathrm{GdTiO}_{3} / \mathrm{SrTiO}_{3}$, being prepared for submission.

3. B. Chen, N. Gauquelin, P. Reith, U. Halisdemir, D. Jannis, M. Spreitzer, M. Huijben, J. Fompeyrine, J. Verbeeck, H. Hilgenkamp, G. Rijnders, G. Koster, Thermal-strain engineered ferromagnetism of $\mathrm{LaMnO}_{3} / \mathrm{SrTiO}_{3}$ heterostructures grown on silicon, being prepared for submission. 


\section{Bibliography}

[1] J. Carlson, Lodestone compass: Chinese or Olmec primacy?, Science volume 189, 753, 1975.

[2] L. Shu-hua, Origine de la boussole II. Aimant et boussole, Isis volume 45, 175, 1954.

[3] J. Hook and H. Hall, Solid State Physics, John Wiley \& Sons, 2nd edition, 1991.

[4] V. Schmidt, The Physics of Superconductors, Springer-Verlag, Berlin, 1st edition, 1997.

[5] M. B. Ketchen and J. R. Kirtley, Design and performance aspects of pickup loop structures for miniature SQUID magnetometry, IEEE Transactions on Applied Superconductivity volume 5, 2133, 1995.

[6] E. H. Brandt, Thin superconductors and SQUIDs in perpendicular magnetic field, Physical Review B volume 72, 024529, 2005.

[7] J. R. Kirtley, L. Paulius et al., Scanning SQUID susceptometers with sub-micron spatial resolution, Review of Scientific Instruments volume 87, 2016.

[8] J. R. Kirtley, M. B. Ketchen et al., High-resolution scanning SQUID microscope, Applied Physics Letters volume 66, 1138, 1995.

[9] P. Reith, X. Renshaw Wang, and H. Hilgenkamp, Analysing magnetism using scanning SQUID microscopy, Review of Scientific Instruments volume 88, 123706, 2017. 
[10] P. Reith, Imaging ferromagnetism on the micrometre scale: a scanning SQUID microscopy study, Master's thesis, University of Twente, Enschede, 2015.

[11] R. H. Koch, D. P. DiVincenzo, and J. Clarke, Model for $1 /$ f flux noise in SQUIDs and qubits, Physical Review Letters volume 98, 267003, 2007.

[12] R. C. Bialczak, R. McDermott et al., 1/f Flux noise in Josephson phase qubits, Physical Review Letters volume 99, 187006, 2007.

[13] P. Dutta, P. Dimon, and P. M. Horn, Energy scales for noise processes in metals, Physical Review Letters volume 43, 646, 1979.

[14] P. Dutta and P. M. Horn, Low-frequency fluctuations in solids: 1/f noise, Reviews of Modern Physics volume 53, 497, 1981.

[15] M. J. Ferrari, M. Johnson et al., Magnetic flux noise in copper oxide superconductors, Journal of Low Temperature Physics volume 94, 15, 1994.

[16] L. Faoro and L. B. Ioffe, Microscopic origin of low-frequency flux noise in Josephson circuits, Physical Review Letters volume 100, 227005, 2008 .

[17] W. S. Choi, Z. Marton et al., Effects of oxygen-reducing atmosphere annealing on $\mathrm{LaMnO}_{3}$ epitaxial thin films, Journal of Physics D: Applied Physics volume 42, 165401, 2009.

[18] F. Rogers, A device for experimental observation of flux vortices trapped in superconducting thin films, Ph.D. thesis, Massachusetts Institute of Technology, 1983.

[19] B. L. Plourde, D. J. Van Harlingen et al., Vortex distributions near surface steps observed by scanning SQUID microscopy, Physical Review B volume 66, 054529, 2002.

[20] N. Kokubo, S. Okayasu et al., Scanning SQUID microscope study of vortex polygons and shells in weak-pinning disks of an amorphous superconducting film, Physical Review B volume 82, 014501, 2010. 
[21] L. Embon, Y. Anahory et al., Probing dynamics and pinning of single vortices in superconductors at nanometer scales, Scientific Reports volume 5, 1, 2015 .

[22] C. W. Hicks, T. M. Lippman et al., Evidence for a nodal energy gap in the iron-pnictide superconductor LaFePO from penetration depth measurements by scanning SQUID susceptometry, Physical Review Letters volume 103, 127003, 2009.

[23] T. Nishio, V. H. Dao et al., Scanning SQUID microscopy of vortex clusters in multiband superconductors, Physical Review B - Condensed Matter and Materials Physics volume 81, 18, 2010.

[24] B. Kalisky, J. R. Kirtley et al., Behavior of vortices near twin boundaries in underdoped $\mathrm{Ba}\left(\mathrm{Fe}_{1-x} \mathrm{Co}_{x}\right)_{2} A s_{2}$, Physical Review B volume 83, 064511, 2011.

[25] B. W. Gardner, J. C. Wynn et al., Manipulation of single vortices in $\mathrm{YBa}_{2} \mathrm{Cu}_{3} \mathrm{O}_{6.354}$ with a locally applied magnetic field, Applied Physics Letters volume 80, 1010, 2002.

[26] H. T. Huy, H. Shishido et al., Direct observation of vortices by scanning SQUID microscope on small superconducting $\mathrm{Mo}_{80} \mathrm{Ge}_{20}$ circular disks, Physica C: Superconductivity and its Applications volume 484, 86, 2013.

[27] A. Kremen, S. Wissberg et al., Mechanical control of individual superconducting vortices, Nano Letters volume 16, 1626, 2016.

[28] T. Nishio, S. Okayasu et al., Penetration of vortices into microsuperconductors observed with a scanning SQUID microscope, Physica C: Superconductivity and its Applications volume 412-414, 379, 2004.

[29] B. L. Plourde, D. J. Van Harlingen et al., Vortex dynamics in thin superconducting strips observed by scanning SQUID microscopy, Physica C: Superconductivity and its Applications volume 341-348, 1023, 2000 .

[30] N. Kokubo, S. Okayasu et al., Direct imaging of vortex polygons and vortex shells in mesoscopic squares of a weak pinning superconducting thin film, Journal of the Physical Society of Japan volume 83, 10, 2014. 
[31] F. S. Wells, A. V. Pan et al., Analysis of low-field isotropic vortex glass containing vortex groups in $\mathrm{YBa}_{2} \mathrm{Cu}_{3} \mathrm{O}_{7_{-x}}$ thin films visualized by scanning SQUID microscopy, Scientific Reports volume 5, 8677, 2015 .

[32] C. Veauvy, K. Hasselbach, and D. Mailly, Micro-SQUID microscopy of vortices in a perforated superconducting Al film, Physical Review B volume 70, 214513, 2004.

[33] C. C. Tsuei, J. R. Kirtley et al., Pairing symmetry and flux quantization in a tricrystal superconducting ring of $\mathrm{YBa}_{2} \mathrm{Cu}_{3} \mathrm{O}_{7_{-} \delta}$, Physical Review Letters volume 73, 593, 1994.

[34] J. Goryo, Vortex with fractional quantum numbers in a chiral p-wave superconductor, Physical Review B volume 61, 4222, 2000.

[35] J. Garaud and E. Babaev, Skyrmionic state and stable half-quantum vortices in chiral p-wave superconductors, Physical Review B volume 86, 060514, 2012.

[36] V. O. Dolocan, C. Veauvy et al., Observation of vortex coalescence in the anisotropic spin-triplet superconductor $\mathrm{Sr}_{2} \mathrm{Ru} \mathrm{O}_{4}$, Physical Review Letters volume 95, 097004, 2005.

[37] S. B. Chung, D. F. Agterberg, and E. A. Kim, Fractional vortex lattice structures in spin-triplet superconductors, New Journal of Physics volume 11, 2009.

[38] G. Carneiro and E. H. Brandt, Vortex lines in films: Fields and interactions, Physical Review B volume 61, 6370, 2000.

[39] D. Agassi and J. R. Cullen, Single-vortex structure in a superconductor film and strip, Physica C: Superconductivity and its Applications volume 334, 274, 2000.

[40] B. J. Roth, N. G. Sepulveda, and J. P. Wikswo, Using a magnetometer to image a two-dimensional current distribution, Journal of Applied Physics volume 65, 361, 1989.

[41] M. Hayashi, T. Kaiwa et al., On mathematical methods to improve imaging of vortices using scanning superconducting quantum interferometer device (SQUID) microscope, Physica C: Superconductivity and its Applications volume 468, 801, 2008. 
[42] J. G. Bednorz and K. A. Miiller, Possible high-T $T_{c}$ superconductivity in the Ba-La-Cu-O system, Zeitschrift für Physik B - Condensed Matter volume 64, 189, 1986.

[43] A. P. Drozdov, P. P. Kong et al., Superconductivity at $250 \mathrm{~K}$ in lanthanum hydride under high pressures, Nature volume 569, 528, 2019.

[44] J. Zaanen, S. Chakravarty et al., Towards a complete theory of high $T_{c}$, Nature Physics volume 2, 138, 2006.

[45] T. Ortlepp, O. Mielke et al., RSFQ circuitry using intrinsic-phase shifts, IEEE Transactions on Applied Superconductivity volume 17, 659, 2007.

[46] A. Finnila, J. Doll et al., Quantum annealing: A new method for minimizing multidimensional functions, Chemical Physics Letters volume 219, 343, 1993.

[47] T. Kadowaki and H. Nishimori, Quantum annealing in the transverse Ising model, Physical Review E volume 58, 5355, 1998.

[48] M. W. Johnson, M. H. Amin et al., Quantum annealing with manufactured spins, Nature volume 473, 194, 2011.

[49] D. A. Wollman, D. J. Van Harlingen et al., Evidence for $d_{x^{2}-y^{2}}$ pairing from the magnetic field modulation of $\mathrm{YBa}_{2} \mathrm{Cu}_{3} \mathrm{O}_{7}-\mathrm{Pb}$ Josephson junctions, Physical Review Letters volume 74, 797, 1995.

[50] J. R. Kirtley, C. C. Tsuei et al., Angle-resolved phase-sensitive determination of the in-plane symmetry in $\mathrm{YBa}_{2} \mathrm{Cu}_{3} \mathrm{O}_{7-\delta}$, Nature Physics volume 2, 353, 2006.

[51] M. Sigrist and M. Rice, Unusual paramagnetic phenomena in granular high-temperature superconductors - A consequence of d-wave pairing?, Reviews of Modern Physics volume 67, 1995.

[52] H. J. Smilde, H. Hilgenkamp et al., $Y$-Ba-Cu-O/Au/Nb ramp-type Josephson junctions, IEEE Transactions on Applied Superconductivity volume 11, 501, 2001.

[53] M. I. Faley, S. B. Mi et al., Multilayer buffer for high-temperature superconductor devices on $\mathrm{MgO}$, Applied Physics Letters volume 89, $3,2006$. 
[54] V. A. Seleznev, M. A. Tarkhov et al., Deposition and characterization of few-nanometers-thick superconducting Mo-Re films, Superconductor Science and Technology volume 21, 2008.

[55] S. Sundar, L. Sharath Chandra et al., Electrical transport and magnetic properties of superconducting $\mathrm{Mo}_{52} \mathrm{Re}_{48}$ alloy, AIP Conference Proceedings volume 1512, 1092, 2013.

[56] M. Aziz, D. Christopher Hudson, and S. Russo, Molybdenum-rhenium superconducting suspended nanostructures, Applied Physics Letters volume 104, 233102, 2014.

[57] C. S. Owen and D. J. Scalapino, Vortex structure and critical currents in Josephson junctions, Physical Review volume 164, 538, 1967.

[58] S. Basavaiaht and R. F. Broom, Characteristics of in-line Josephson tunneling gates, IEEE Transactions on Magnetics volume 11, 759, 1975 .

[59] C. Alexiou, R. Jurgons et al., Medical applications of magnetic nanoparticles, Frontiers of Nanoscience volume 6, 217, 2014.

[60] A. Ito, M. Shinkai et al., Medical application of functionalized magnetic nanoparticles, Journal of Bioscience and Bioengineering volume 100, 1, 2005.

[61] P. Terzieff and K. L. Komarek, The antiferromagnetic and ferrimagnetic properties of iron selenides with NiAs-type structure, Monatshefte für Chemie volume 109, 1037, 1978.

[62] H. Zhang, G. Long et al., $\mathrm{Fe}_{3} \mathrm{Se}_{4}$ nanostructures with giant coercivity synthesized by solution chemistry, Chemistry of Materials volume 23, 3769, 2011.

[63] I. S. Lyubutin, C. R. Lin et al., Structural, magnetic, and electronic properties of iron selenide $\mathrm{Fe}_{6-7} \mathrm{Se}_{8}$ nanoparticles obtained by thermal decomposition in high-temperature organic solvents, Journal of Chemical Physics volume 141, 2014.

[64] R. Oliveira-Silva, R. A. Pereira et al., Temperature-responsive nanomagnetic logic gates for cellular hyperthermia, Materials Horizons volume 6, 524, 2019. 
[65] G. Long, H. Zhang et al., Magnetic anisotropy and coercivity of $\mathrm{Fe}_{3} \mathrm{Se}_{4}$ nanostructures, Applied Physics Letters volume 99, 202103, 2011.

[66] D. Singh, S. K. Gupta et al., Prediction of new multiferroic and magnetoelectric material $\mathrm{Fe}_{3} \mathrm{Se}_{4}$, arXiv 1805.10607, 2018.

[67] L. Néel, Théorie du traînage magnétique des ferromagnétiques en grains fins avec application aux terres cuites, Annales de Géophysique volume 5, 99, 1949.

[68] L. Néel, Some theoretical aspects of rock-magnetism, Advances in Physics volume 4, 191, 1955.

[69] T. Berndt, A. R. Muxworthy, and K. Fabian, Does size matter? Statistical limits of paleomagnetic field reconstruction from small rock specimens, Journal of Geophysical Research: Solid Earth volume 121, 15, 2016.

[70] E. Thellier and O. Thellier, Sur l'intensité du champ magné tique terrestre dans le passé historique et géologique, Annales de Géophysique volume 15, 285, 1959.

[71] L. V. De Groot, K. Fabian et al., Magnetic force microscopy reveals meta-stable magnetic domain states that prevent reliable absolute palaeointensity experiments, Nature Communications volume 5, 4548, 2014.

[72] K. Fabian and L. V. de Groot, A uniqueness theorem in potential theory with implications for tomography-assisted inversion, arXiv 1712.06136, 2017.

[73] R. Hartstra, Grain-size dependence of initial susceptibility and saturation magnetization-related parameters of four natural magnetites in the PSD-MD range, Geophysical Journal International volume 71, 477, 1982.

[74] F. Walz, The Verwey transition - a topical review, Journal of Physics: Condensed Matter volume 14, R285, 2002.

[75] A. R. Muxworthy, D. J. Dunlop, and Ö. Özdemir, Low-temperature cycling of isothermal and anhysteretic remanence: microcoercivity and 
magnetic memory, Earth and Planetary Science Letters volume 205, $173,2003$.

[76] A. R. Muxworthy and E. McClelland, The causes of low-temperature demagnetization of remanence in multidomain magnetite, Geophysical Journal International volume 140, 115, 2000.

[77] M. Kustov, P. Laczkowski et al., Magnetic characterization of micropatterned $\mathrm{Nd}-\mathrm{Fe}-\mathrm{B}$ hard magnetic films using scanning Hall probe microscopy, Journal of Applied Physics volume 108, 2010.

[78] P. Maletinsky, S. Hong et al., A robust scanning diamond sensor for nanoscale imaging with single nitrogen-vacancy centres, Nature Nanotechnology volume 7, 320, 2012.

[79] E. A. Lima, A. C. Bruno et al., Scanning magnetic tunnel junction microscope for high-resolution imaging of remanent magnetization fields, Measurement Science and Technology volume 25, 2014.

[80] A. Ramirez, Colossal magnetoresistance, Journal of Physics Condensed Matter volume 9, 8171, 1997.

[81] Y. K. Liu, Y. W. Yin, and X. G. Li, Colossal magnetoresistance in manganites and related prototype devices, Chinese Physics B volume 22, 087502, 2013.

[82] A. Gupta, T. R. McGuire et al., Growth and giant magnetoresistance properties of La-deficient $L a_{x} M n O_{3-\delta}(0.67 \leq x \leq 1)$ films, Applied Physics Letters volume 67, 3494, 1995.

[83] X. R. Wang, C. J. Li et al., Imaging and control of ferromagnetism in $\mathrm{LaMnO}_{3} / \mathrm{SrTiO}_{3}$ heterostructures, Science volume 349, 716, 2015.

[84] I. Marozau, P. T. Das et al., Influence of La and Mn vacancies on the electronic and magnetic properties of $\mathrm{LaMnO}_{3}$ thin films grown by pulsed laser deposition, Physical Review B volume 89, 174422, 2014.

[85] Z. Marton, S. S. Sung et al., Growth control of stoichiometry in $\mathrm{LaMnO}_{3}$ epitaxial thin films by pulsed laser deposition, Journal of Crystal Growth volume 312, 2923, 2010. 
[86] Y. G. Zhao, M. Rajeswari et al., Effect of oxygen content on the structural, transport, and magnetic properties of $L a_{1-\delta} M n_{1-\delta} O_{3}$ thin films, Journal of Applied Physics volume 86, 6327, 1999.

[87] Y. Anahory, L. Embon et al., Emergent nanoscale superparamagnetism at oxide interfaces, Nature Communications volume 7, 12566, 2016.

[88] Z. Chen, Z. Chen et al., Electron accumulation and emergent magnetism in $\mathrm{LaMnO}_{3} / \mathrm{SrTiO}_{3}$ heterostructures, Physical Review Letters volume 119, 1, 2017.

[89] R. Zhao, K. Jin et al., The oxygen vacancy effect on the magnetic property of the $\mathrm{LaMnO}_{3-\delta}$ thin films, Applied Physics Letters volume 102, 122402, 2013.

[90] W. Niu, W. Liu et al., Direct demonstration of the emergent magnetism resulting from the multivalence $\mathrm{Mn}$ in a $\mathrm{LaMnO}_{3}$ epitaxial thin film system, Advanced Electronic Materials volume 4, 1800055, 2018.

[91] J. Roqueta, A. Pomar et al., Strain-engineered ferromagnetism in $\mathrm{LaMnO}_{3}$ thin films, Crystal Growth and Design volume 15, 5332, 2015.

[92] H. S. Kim and H. M. Christen, Controlling the magnetic properties of $\mathrm{LaMnO}_{3}$ thin films on $\mathrm{SrTiO}_{3}$ (100) by deposition in a $\mathrm{O}_{2} / \mathrm{Ar}$ gas mixture, Journal of Physics Condensed Matter volume 22, 146007, 2010.

[93] N. Nakagawa, H. Y. Hwang, and D. A. Muller, Why some interfaces cannot be sharp, Nature Materials volume 5, 204, 2006.

[94] Z. Zhong and P. Hansmann, Band alignment and charge transfer in complex oxide interfaces, Physical Review X volume 7, 011023, 2017.

[95] L. Wu, C. Li et al., Interface-induced enhancement of ferromagnetism in insulating $\mathrm{LaMnO}_{3}$ ultrathin films, ACS Applied Materials and Interfaces volume 9, 44931, 2017.

[96] H. Fujishiro, T. Fukase, and M. Ikebe, Charge ordering and sound velocity anomaly in $\mathrm{La}_{1-X} \mathrm{Sr}_{X} \mathrm{MnO}_{3}(X \geq 0.5)$, Journal of the Physical Society of Japan volume 67, 2582, 1998. 
[97] E. Dagotto, T. Hotta, and A. Moreo, Colossal magnetoresistant materials: The key role of phase separation, Physics Reports volume 344, $1,2001$.

[98] J. A. Mundy, Y. Hikita et al., Visualizing the interfacial evolution from charge compensation to metallic screening across the manganite metal-insulator transition, Nature Communications volume 5, 3464, 2014 .

[99] K. H. Ahn and A. J. Millis, Effects of uniaxial strain in $\mathrm{LaMnO}_{3}$, Physical Review B volume 64, 115103, 2001.

[100] B. R. K. Nanda and S. Satpathy, Magnetic and orbital order in $\mathrm{LaMnO}_{3}$ under uniaxial strain: A model study, Physical Review B volume 81, 174423, 2010.

[101] B. R. Nanda and S. Satpathy, Density functional studies of $\mathrm{LaMnO}_{3}$ under uniaxial strain, Journal of Magnetism and Magnetic Materials volume 322, 3653, 2010.

[102] J. H. Lee, K. T. Delaney et al., Strong coupling of Jahn-Teller distortion to oxygen-octahedron rotation and functional properties in epitaxially strained orthorhombic $\mathrm{LaMnO}_{3}$, Physical Review B volume 88, 174426, 2013.

[103] M. An, Y. Weng et al., Appearance and disappearance of ferromagnetism in ultrathin $\mathrm{LaMnO}_{3}$ on $\mathrm{SrTiO}_{3}$ substrate: A viewpoint from first principles, Physical Review B volume 96, 235112, 2017.

[104] C. Zhong, X. Lu et al., Strain-induced insulating ferromagnetism in $\mathrm{LaMnO}_{3}$ thin films from first-principles investigations, Journal of Magnetism and Magnetic Materials volume 466, 406, 2018.

[105] G. J. H. M. Rijnders, H. Rogalla et al., Quasi-ideal strontium titanate crystal surfaces through formation of strontium hydroxide, Applied Physics Letters volume 73, 2920, 2002.

[106] A. N. Vasiliev, O. S. Volkova et al., Valence states and metamagnetic phase transition in partially B-site-disordered perovskite $E u M n_{0.5} \mathrm{Co}_{0.5} \mathrm{O}_{3}$, Physical Review B volume 77, 104442, 2008. 
[107] V. R. Galakhov, M. Demeter et al., Mn $3 s$ exchange splitting in mixed-valence manganites, Physical Review B volume 65, 113102, 2002.

[108] S. H. Estemirova, A. V. Fetisov, and V. B. Fetisov, X-ray photoelectron spectroscopy study of the electronic structure of $\mathrm{La}_{1-x} \mathrm{Ca}_{x} \mathrm{Mn} \mathrm{O}_{3+\delta}$ solid solutions, Journal of Applied Spectroscopy volume 76, 394, 2009.

[109] A. Santoni, G. Speranza et al., Core-level and valence band photoemission study of $\mathrm{La}_{1-x} \mathrm{Sr}_{x} \mathrm{MnO}_{3}$ perovskite oxide powders synthesized by mechanically and thermally activated solid-state reaction, Journal of Physics Condensed Matter volume 11, 3387, 1999.

[110] T. Saitoh, a. E. Bocquet et al., Electronic structure of $\mathrm{La}_{1-\mathrm{x}} \mathrm{Sr}_{x} \mathrm{MnO}_{3}$ studied by photoemission and $x$-ray-absorption spectroscopy, Physical Review B volume 51, 13942, 1995.

[111] N. Ashcroft and N. Mermin, Solid state physics, Brooks/Cole, Belmont, CA, 1st edition, 1976.

[112] B. T. Thole, P. Carra et al., X-ray circular dichroism as a probe of orbital magnetization, Physical Review Letters volume 68, 1943, 1992.

[113] P. Carra, H. König et al., Magnetic X-ray dichroism, Physica B: Condensed Matter volume 192, 182, 1993.

[114] J. J. Peng, C. Song et al., Restoring the magnetism of ultrathin $\mathrm{LaMnO}_{3}$ films by surface symmetry engineering, Physical Review B volume 94, 214404, 2016.

[115] R. P. Borges, W. Guichard et al., Magnetic and electric "dead" layers in $\left(\mathrm{La}_{0.7} \mathrm{Sr}_{0.3}\right) \mathrm{MnO}_{3}$ thin films, Journal of Applied Physics volume 89, 3868, 2001.

[116] H. Yamada, Y. Ishii et al., Engineered interface of magnetic oxides, Science volume 305, 646, 2004.

[117] Y. H. Sun, Y. G. Zhao et al., Electric and magnetic modulation of fully strained dead layers in $\mathrm{La}_{0.67} \mathrm{Sr}_{0.33} \mathrm{MnO}_{3}$ films, Physical Review B volume 78, 024412, 2008. 
[118] M. Huijben, L. W. Martin et al., Critical thickness and orbital ordering in ultrathin $\mathrm{La}_{0.7} \mathrm{Sr}_{0.3} \mathrm{MnO}_{3}$ films, Physical Review B volume 78, 094413, 2008.

[119] S. B. Porter, M. Venkatesan et al., Magnetic dead layers in $\mathrm{La}_{0.7} \mathrm{Sr}_{0.3} \mathrm{MnO}_{3}$ revisited, IEEE Transactions on Magnetics volume 53, 6000904, 2017.

[120] N. Mottaghi, M. S. Seehra et al., Insights into the magnetic dead layer in $\mathrm{La}_{0.7} \mathrm{Sr}_{0.3} \mathrm{MnO}_{3}$ thin films from temperature, magnetic field and thickness dependence of their magnetization, AIP Advances volume 8, 056319, 2018.

[121] M. P. de Jong, I. Bergenti et al., Evidence for $\mathrm{Mn}^{2+}$ ions at surfaces of $\mathrm{La}_{0.7} \mathrm{Sr}_{0.3} \mathrm{MnO}_{3}$ thin films, Physical Review B volume 71, 014434, 2005.

[122] R. Peng, H. C. Xu et al., Tuning the dead-layer behavior of $\mathrm{La}_{0.67} \mathrm{Sr}_{0.33} \mathrm{MnO}_{3} / \mathrm{SrTiO}_{3}$ via interfacial engineering, Applied Physics Letters volume 104, 081606, 2014.

[123] C. Ritter, M. R. Ibarra et al., Influence of oxygen content on the structural, magnetotransport, and magnetic properties of $\mathrm{LaMnO}_{3+\delta}$, Physical Review B volume 56, 8902, 1997.

[124] L. Yu and A. Zunger, A polarity-induced defect mechanism for conductivity and magnetism at polar-nonpolar oxide interfaces, Nature Communications volume 5, 5118, 2014.

[125] Y. Z. Chen, F. Trier et al., Extreme mobility enhancement of two-dimensional electron gases at oxide interfaces by charge-transferinduced modulation doping, Nature Materials volume 14, 801, 2015.

[126] D. Lu, D. J. Baek et al., Synthesis of freestanding single-crystal perovskite films and heterostructures by etching of sacrificial watersoluble layers, Nature Materials volume 15, 1255, 2016.

[127] S. Sae Hong, H. Y. Hwang et al., Two-dimensional limit of crystalline order in perovskite membrane films, Science Advances volume 3, eaao5173, 2017. 
[128] D. Ji, S. Cai et al., Freestanding crystalline oxide perovskites down to the monolayer limit, Nature volume 570, 87, 2019.

[129] H. Liu and X. Yang, A brief review on perovskite multiferroics, Ferroelectrics volume 507, 69, 2017.

[130] H. Y. Hwang, Y. Iwasa et al., Emergent phenomena at oxide interfaces, Nature Materials volume 11, 103, 2012.

[131] J. G. Bednorz and K. A. Müller, Perovskite-type oxides-The new approach to high- $T_{c}$ superconductivity, Reviews of Modern Physics volume 60, 585, 1988.

[132] D. G. Schlom, L. Q. Chen et al., A thin film approach to engineering functionality into oxides, Journal of the American Ceramic Society volume 91, 2429, 2008.

[133] A. Ohtomo and H. Y. Hwang, A high-mobility electron gas at the LAO/STO heterointerface., Nature volume 427, 423, 2004.

[134] S. Thiel, G. Hammerl et al., Tunable quasi-two-dimensional electron gases in oxide heterostructures, Science volume 905, 1942, 2006.

[135] H. Chen, A. M. Kolpak, and S. Ismail-Beigi, Electronic and magnetic properties of $\mathrm{SrTiO}_{3} / \mathrm{LaAlO}_{3}$ interfaces from first principles, Advanced Materials volume 22, 2881, 2010.

[136] Y. S. Hou, H. J. Xiang, and X. G. Gong, Intrinsic insulating ferromagnetism in manganese oxide thin films, Physical Review B volume 89, 064415, 2014.

[137] B. Kalisky, J. A. Bert et al., Critical thickness for ferromagnetism in $\mathrm{LaAlO}_{3} / \mathrm{SrTiO}_{3}$ heterostructures, Nature Communications volume 3, 922, 2012.

[138] R. Pentcheva, M. Huijben et al., Parallel electron-hole bilayer conductivity from electronic interface reconstruction, Physical Review Letters volume 104, 166804, 2010.

[139] A. Brinkman, M. Huijben et al., Magnetic effects at the interface between non-magnetic oxides, Nature Materials volume 6, 493, 2007. 
[140] Ariando, X. Wang et al., Electronic phase separation at the $\mathrm{LaAlO}_{3} / \mathrm{SrTiO}_{3}$ interface, Nature Communications volume 2, 188, 2011.

[141] J. M. Coey, M. Venkatesan, and P. Stamenov, Surface magnetism of strontium titanate, Journal of Physics: Condensed Matter volume 28, 485001, 2016.

[142] N. Pavlenko, T. Kopp et al., Magnetic and superconducting phases at the $\mathrm{LaAlO}_{3} / \mathrm{SrTiO}_{3}$ interface: The role of interfacial Ti $3 d$ electrons, Physical Review B - Condensed Matter and Materials Physics volume 85, 1, 2012.

[143] E. Lesne, N. Reyren et al., Suppression of the critical thickness threshold for conductivity at the $\mathrm{LaAlO}_{3} / \mathrm{SrTiO}_{3}$ interface, Nature Communications volume 5, 4291, 2014.

[144] D. C. Vaz, E. Lesne et al., Tuning up or down the critical thickness in $\mathrm{LaAlO}_{3} / \mathrm{SrTiO}_{3}$ through in situ deposition of metal overlayers, Advanced Materials volume 29, 1700486, 2017.

[145] Y. J. Shi, S. Wang et al., Tuning the carrier density of $\mathrm{LaAlO}_{3}$ $/ \mathrm{SrTiO}_{3}$ interfaces by capping $\mathrm{La}_{1-x} \mathrm{Sr}_{x} \mathrm{MnO}_{3}$, Applied Physics Letters volume 102, 071605, 2013.

[146] M. Huijben, G. Koster et al., Defect engineering in oxide heterostructures by enhanced oxygen surface exchange, Advanced Functional Materials volume 23, 5240, 2013.

[147] K. A. Stoerzinger, M. Risch et al., Recent insights into manganese oxides in catalyzing oxygen reduction kinetics, ACS Catalysis volume 5 , 6021, 2015.

[148] P. R. Willmott, S. A. Pauli et al., Structural basis for the conducting interface between $\mathrm{LaAlO}_{3}$ and $\mathrm{SrTiO}_{3}$, Physical Review Letters volume 99, 155502, 2007.

[149] S. A. Chambers, M. H. Engelhard et al., Instability, intermixing and electronic structure at the epitaxial $\mathrm{LaAlO}_{3} / \mathrm{SrTiO}_{3}(001)$ heterojunction, Surface Science Reports volume 65, 317, 2010. 
[150] L. Qiao, T. C. Droubay et al., Thermodynamic instability at the stoichiometric $\mathrm{LaAlO}_{3} / \mathrm{SrTiO}_{3}$ (001) interface, Journal of Physics Condensed Matter volume 22, 312201, 2010.

[151] C. Cantoni, J. Gazquez et al., Electron transfer and ionic displacements at the origin of the $2 D$ electron gas at the LAO/STO interface: Direct measurements with atomic-column spatial resolution, Advanced Materials volume 24, 3952, 2012.

[152] A. D. Caviglia, S. Gariglio et al., Electric field control of the $\mathrm{LaAlO}_{3} / \mathrm{SrTiO}_{3}$ interface ground state, Nature volume 456, 624, 2008.

[153] C. Bell, S. Harashima et al., Dominant mobility modulation by the electric field effect at the $\mathrm{LaAlO}_{3} / \mathrm{SrTiO}_{3}$ interface, Physical Review Letters volume 103, 25, 2009.

[154] A. D. Caviglia, M. Gabay et al., Tunable Rashba spin-orbit interaction at oxide interfaces, Physical Review Letters volume 104, 126803, 2010 .

[155] A. Smink, Manifold field effects at a complex oxide interface, Ph.D. thesis, University of Twente, 2019.

[156] D. Griffiths, Introduction to electrodynamics, Prentice-Hall, Inc., Englewood Cliffs, NJ, 3rd edition, 1999.

[157] A. Finkler, Y. Segev et al., Self-aligned nanoscale SQUID on a tip, Nano Letters volume 10, 1046, 2010.

[158] D. Halbertal, J. Cuppens et al., Nanoscale thermal imaging of dissipation in quantum systems, Nature volume 539, 407, 2016.

[159] D. Hsieh, D. Qian et al., A topological Dirac insulator in a quantum spin Hall phase, Nature volume 452, 970, 2008.

[160] J. R. Kirtley, C. Kallin et al., Upper limit on spontaneous supercurrents in $\mathrm{Sr}_{2} \mathrm{RuO}_{4}$, Physical Review B volume 76, 014526, 2007.

[161] C. W. Hicks, J. R. Kirtley et al., Limits on superconductivity-related magnetization in $\mathrm{Sr}_{2} \mathrm{RuO}_{4}$ and $\mathrm{PrOs}_{4} \mathrm{Sb}_{12}$ from scanning SQUID microscopy, Physical Review B volume 81, 214501, 2010. 
[162] M. Sato and Y. Ando, Topological superconductors: A review, Reports on Progress in Physics volume 80, 076501, 2017.

[163] Y. Asano, Y. Tanaka et al., Josephson interferometer in a ring topology as a proof of the symmetry of $\mathrm{Sr}_{2} \mathrm{RuO}_{4}$, Physical Review B - Condensed Matter and Materials Physics volume 71, 1, 2005.

[164] M. Matsumoto and M. Sigrist, Quasiparticle states near the surface and the domain wall in a $p_{x} \pm i p_{y}$-wave superconductor, Journal of the Physical Society of Japan volume 68, 994, 1999.

[165] P. G. Björnsson, Y. Maeno et al., Scanning magnetic imaging of $\mathrm{Sr}_{2} \mathrm{RuO}_{4}$, Physical Review B volume 72, 012504, 2005.

[166] M. Ichioka, K. Machida, and J. A. Sauls, Vortex states of chiral pwave superconductors, Journal of Physics: Conference Series volume 400, 022031, 2012.

[167] J. Garaud and E. Babaev, Properties of skyrmions and multi-quanta vortices in chiral p-wave superconductors, Scientific Reports volume 5, 17540, 2015.

[168] J. Garaud, E. Babaev et al., Lattices of double-quanta vortices and chirality inversion in $p_{x}+i p_{y}$ superconductors, Physical Review B volume 94, 104509, 2016.

[169] T. Riseman, P. Kealey et al., Observation of a square flux-line lattice in the unconventional superconductor $\mathrm{Sr}_{2} \mathrm{RuO}_{4}$, Nature volume 396, $242,1998$.

[170] C. M. Aegerter, S. H. Lloyd et al., Evidence for a square vortex lattice in $\mathrm{Sr}_{2} \mathrm{Ru} \mathrm{O}_{4}$ from muon-spin-rotation measurements, Journal of Physics Condensed Matter volume 10, 7445, 1998.

[171] S. J. Ray, A. S. Gibbs et al., Muon-spin rotation measurements of the vortex state in $\mathrm{Sr}_{2} \mathrm{RuO}_{4}$ : Type-1.5 superconductivity, vortex clustering, and a crossover from a triangular to a square vortex lattice, Physical Review B volume 89, 094504, 2014.

[172] L. Kramer and W. Pesch, Core structure and low-energy spectrum of isolated vortex lines in clean superconductors at $T \ll T_{c}$, Zeitschrift für Physik volume 269, 59, 1974. 
[173] Y. Kato and N. Hayashi, Kramer-Pesch effect in chiral p-wave superconductors, Journal of the Physical Society of Japan volume 70, 3368, 2001.

[174] N. Hayashi, N. Kurosawa et al., Effect of Born and unitary impurity scattering on the Kramer-Pesch shrinkage of a vortex core in a chiral p-wave superconductor, Physica C: Superconductivity and its Applications volume 494, 131, 2013.

[175] D. J. P. Morris, D. A. Tennant et al., Dirac strings and magnetic monopoles in the spin ice $\mathrm{Dy}_{2} \mathrm{Ti}_{2} \mathrm{O}_{7}$, Science volume 326, 411, 2009.

[176] L. Landau and E. Lifshitz, Electrodynamics of continuous media, Pergamon Press, Oxford, 2nd edition, 2013.

[177] X.-L. Qi, R. Li et al., Inducing a Magnetic Monopole, Science volume 323, 1184, 2009.

[178] D. I. Khomskii, Magnetic monopoles and unusual dynamics of magnetoelectrics, Nature Communications volume 5, 4793, 2014.

[179] M. Fechner, N. A. Spaldin, and I. E. Dzyaloshinskii, Magnetic field generated by a charge in a uniaxial magnetoelectric material, Physical Review B volume 89, 184415, 2014.

[180] Q. N. Meier, M. Fechner et al., Search for the magnetic monopole at a magnetoelectric surface, Physical Review X volume 9, 11011, 2019.

[181] E. M. Spanton, A. J. Rosenberg et al., Electric coupling in scanning SQUID measurements, arXiv 1512.03373, 2015.

[182] L. Fu and C. L. Kane, Superconducting proximity effect and majorana fermions at the surface of a topological insulator., Physical Review Letters volume 100, 096407, 2008.

[183] I. Sochnikov, A. J. Bestwick et al., Direct measurement of currentphase relations in superconductor/topological insulator/superconductor junctions, Nano Letters volume 13, 3086, 2013.

[184] I. Sochnikov, L. Maier et al., Nonsinusoidal current-phase relationship in josephson junctions from the $3 \mathrm{D}$ topological insulator $\mathrm{HgTe}$, Physical Review Letters volume 114, 1, 2015. 
[185] A. M. Bozkurt, B. Pekerten, and I. Adagideli, Work extraction and Landauer's principle in a quantum spin Hall device, Physical Review B volume 97, 245414, 2018.

[186] J. Voerman, Putting a spin on topological matter, Ph.D. thesis, University of Twente, 2019.

[187] J. L. Starck, E. Pantin, and F. Murtagh, Deconvolution in astronomy: A review, Publications of the Astronomical Society of the Pacific volume 114, 1051, 2002.

[188] S. A. Gudoshnikov, Y. V. Deryuzhkina et al., Magnetic flux guide for high-resolution SQUID microscope, IEEE Transactions on Applied Superconductivity volume 11, 219, 2001.

[189] S. Tanaka, K. Matsuda et al., Properties of high-T $T_{c}$ superconducting quantum interference device microscope with high $\mu$-metal needle, Japanese Journal of Applied Physics volume 40, 2001.

[190] T. Nagaishi, K. Minamimura, and H. Itozaki, HTS SQUID microscope head with permalloy flux guide, IEEE Transactions on Applied Superconductivity volume 13, 227, 2003.

[191] M. I. Faley, E. A. Kostyurina et al., Nondestructive evaluation using a high-T $T_{c}$ SQUID microscope, IEEE Transactions on Applied Superconductivity volume 27, 1600905, 2017.

[192] M. Tachiki, T. Hayashi et al., STM-SQUID probe microscope based on an RF SQUID magnetometer, IEEE Transactions on Applied Superconductivity volume 19, 874, 2009.

[193] T. S. Lee, E. Dantsker, and J. Clarke, High-transition temperature superconducting quantum interference device microscope, Review of Scientific Instruments volume 67, 4208, 1996.

[194] J. Dechert, M. Mueck, and C. Heiden, A scanning SQUID microscope for samples at room temperature, IEEE Transactions on Applied Superconductivity volume 9, 4111, 1999.

[195] E. F. Fleet, S. Chatraphorn et al., Closed-cycle refrigerator-cooled scanning SQUID microscope for room-temperature samples, Review of Scientific Instruments volume 72, 3281, 2001. 
[196] R. C. Black, A. Mathai et al., Magnetic microscopy using a liquid nitrogen cooled $\mathrm{YBa}_{2} \mathrm{Cu}_{3} \mathrm{O}_{7}$ superconducting quantum interference device, Applied Physics Letters volume 62, 2128, 1993.

[197] S. Tanaka, O. Yamazaki et al., Windowless high-T $T_{c}$ superconducting quantum interference device microscope, Japanese Journal of Applied Physics volume 38, L505, 1999.

[198] L. Bishop-Van Horn, Z. Cui et al., Cryogen-free variable temperature scanning SQUID microscope, Review of Scientific Instruments volume 90, 063705, 2019.

[199] Y. Shperber, N. Vardi et al., Scanning SQUID microscopy in a cryogen-free cooler, Review of Scientific Instruments volume 90, 053702, 2019.

[200] S. Miyajima, T. Okamoto et al., Vector pickup system customized for scanning SQUID microscopy, IEEE Transactions on Applied Superconductivity volume 25, 1600704, 2015.

[201] T. Vu, T. Ho et al., SQUID microscopy for mapping vector magnetic fields, Superconductor Science and Technology 2019.

[202] M. B. Ketchen and J. R. Kirtley, Miniature vector magnetometer for scanning squid microscopy, IEEE Transactions on Applied Superconductivity volume 7, 3139, 1997. 